Florida International University

FIU Digital Commons

$12-6-2012$

\title{
A Retrospective-Longitudinal Examination of the Relationship between Apportionment of Seat Time in Community-College Algebra Courses and Student Academic Performance
}

Steven M. Roig-Watnik

Florida International University, sroigwatnik@yahoo.com

DOI: $10.25148 /$ etd.FI13042204

Follow this and additional works at: https://digitalcommons.fiu.edu/etd

\section{Recommended Citation}

Roig-Watnik, Steven M., "A Retrospective-Longitudinal Examination of the Relationship between Apportionment of Seat Time in Community-College Algebra Courses and Student Academic Performance" (2012). FIU Electronic Theses and Dissertations. 826.

https://digitalcommons.fiu.edu/etd/826 


\section{FLORIDA INTERNATIONAL UNIVERSITY}

Miami, Florida

\section{A RETROSPECTIVE-LONGITUDINAL EXAMINATION OF THE RELATIONSHIP BETWEEN APPORTIONMENT OF SEAT TIME IN COMMUNITY-COLLEGE ALGEBRA COURSES AND STUDENT ACADEMIC PERFORMANCE}

A dissertation submitted in partial fulfillment of the requirements for the degree of DOCTOR OF EDUCATION

in

HIGHER EDUCATION

by

Steven Michael Roig-Watnik 
To: Dean Delia C. Garcia

College of Education

This dissertation, written by Steven Michael Roig-Watnik, and entitled A RetrospectiveLongitudinal Examination of the Relationship between Apportionment of Seat Time in Community-College Algebra Courses and Student Academic Performance, having been approved in respect to style and intellectual content, is referred to you for judgment.

We have read this dissertation and recommend that it be approved.

Glenda D. Musoba

Thomas G. Reio, Jr.

Marc Weinstein

Benjamin Baez, Major Professor

Date of Defense: December 6, 2012

The dissertation of Steven Michael Roig-Watnik is approved.

Dean Delia C. Garcia

College of Education

Dean Lakshmi N. Reddi

University Graduate School

Florida International University, 2012 
(C) Copyright 2012 by Steven Michael Roig-Watnik

All rights reserved. 


\section{DEDICATION}

With infinite love and gratitude, I dedicate this dissertation...

... To my extraordinary wife and unceasing motivator, Dr. Mattie Roig-Watnik. Without

her steadfast support and sage counsel, this milestone would not have been attainable.

"Thank you for marrying me."

... To my folks, Mimi Watnik and the-late-but-omnipresent-in-spirit Joe Watnik, whose sacrifices, love, acceptance, and humor permeate everything I am and do.

“Wow! How brilliantly I handpicked my parents!”

... And to the teachers/mentors I have had throughout my life who maintained high, uncompromising standards but, even more importantly, vigorously cultivated my intellectual curiosity and instilled in me an ethos of "Question everything! Scrutinize orthodoxy! Avoid echo chambers!” 


\section{ACKNOWLEDGMENTS}

I am truly grateful to the faculty, staff, and administration at Florida International University and, in particular, those within its College of Education for the many learning opportunities they have afforded me. FIU's program has provided what I had been seeking for a long time: an intellectually-rigorous, growth-inducing experience that nurtured mid-career working professionals like me.

I would also like to express my appreciation to the members of my dissertation committee: Dr. Glenda Musoba, Dr. Tom Reio, and Dr. Marc Weinstein. I feel very fortunate to have had the guidance and support of such distinguished scholars and dedicated educators. Dr. Musoba deserves special kudos for her willingness to remain on my committee even during her transition to a tenured faculty position at Texas A\&M. For a recognized researcher of her caliber to display that level of commitment to students speaks volumes about her altruistic character and, for me, serves as a role model to continue to strive to emulate in the years ahead with my own students. And, speaking of stellar support of students, I would be remiss if I did not acknowledge two College of Education all-stars: Dr. Linda Bliss for her detailed feedback and the amiable way she always conveyed it, and Ms. Caprila Almeida for all of her logistical support and the genuine concern for students she exhibits on a daily basis.

By far, my largest debt of gratitude is owed to Dr. Benjamin Baez, my major professor. Without his no-holds-barred candor, staunch advocacy, and belief in my potential, I would never have successfully completed this degree. I deeply admire and respect him on so many levels, both professionally and personally. To any educators/researchers reading this, I strongly recommend reading Ben Baez's scholarly 
works, starting with, in my opinion, his and Deron Boyles' book entitled The Politics of Inquiry: Education Research and the "Culture of Science". It is a seminal accomplishment that needs to be contemplated by all of us who are entranced in this accountability era.

Finally, to my colleagues and my employer, this transformative educational experience would not have been possible without your encouragement and support. I am privileged to work at an institution whose senior leadership and institutional-research staff appreciate the potential institutional value of dissertation-level research and, as such, actively strive to support their faculty/administrators in these types of scholastic pursuits, both financially and in terms of data/resources. 


\begin{abstract}
OF THE DISSERTATION
A RETROSPECTIVE-LONGITUDINAL EXAMINATION OF THE RELATIONSHIP BETWEEN APPORTIONMENT OF SEAT TIME IN COMMUNITY-COLLEGE ALGEBRA COURSES AND STUDENT ACADEMIC PERFORMANCE
\end{abstract} by Steven Michael Roig-Watnik

Florida International University, 2012 Miami, FL Professor Benjamin Baez, Major Professor

During the past decade, there has been a dramatic increase by postsecondary institutions in providing academic programs and course offerings in a multitude of formats and venues (Biemiller, 2009; Kucsera \& Zimmaro, 2010; Lang, 2009; Mangan, 2008). Strategies pertaining to reapportionment of course-delivery seat time have been a major facet of these institutional initiatives; most notably, within many open-door 2-year colleges. Often, these enrollment-management decisions are driven by the desire to increase market-share, optimize the usage of finite facility capacity, and contain costs, especially during these economically turbulent times. So, while enrollments have surged to the point where nearly one in three 18-to-24 year-old U.S. undergraduates are community college students (Pew Research Center, 2009), graduation rates, on average, still remain distressingly low (Complete College America, 2011). Among the learningtheory constructs related to seat-time reapportionment efforts is the cognitive phenomenon commonly referred to as the spacing effect, the degree to which learning is 
enhanced by a series of shorter, separated sessions as opposed to fewer, more massed episodes.

This ex post facto study explored whether seat time in a postsecondary developmental-level algebra course is significantly related to: course success; courseenrollment persistence; and, longitudinally, the time to successfully complete a generaleducation-level mathematics course. Hierarchical logistic regression and discrete-time survival analysis were used to perform a multi-level, multivariable analysis of a student cohort $(N=3,284)$ enrolled at a large, multi-campus, urban community college. The subjects were retrospectively tracked over a 2-year longitudinal period. The study found that students in long seat-time classes tended to withdraw earlier and more often than did their peers in short seat-time classes $(p<.05)$. Additionally, a model comprised of nine statistically significant covariates (all with $p$-values less than .01) was constructed. However, no longitudinal seat-time group differences were detected nor was there sufficient statistical evidence to conclude that seat time was predictive of developmentallevel course success.

A principal aim of this study was to demonstrate - to educational leaders, researchers, and institutional-research/business-intelligence professionals - the advantages and computational practicability of survival analysis, an underused but more powerful way to investigate changes in students over time. 


\section{TABLE OF CONTENTS}

CHAPTER PAGE

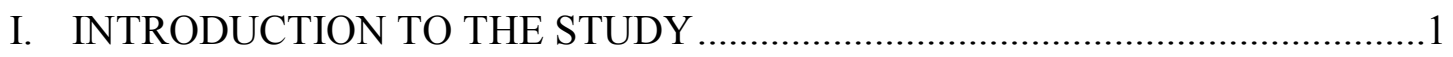

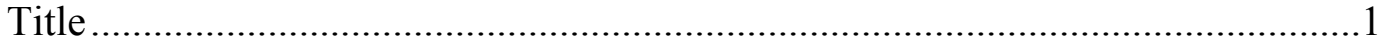

Background to the Problem .........................................................................

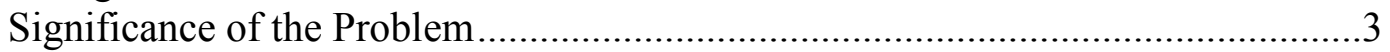

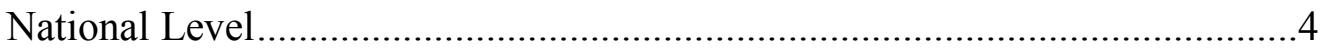

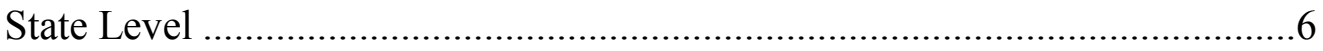

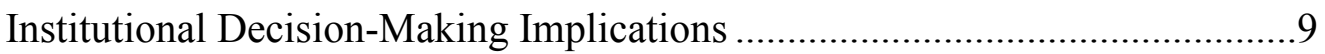

Statement of the Problem.............................................................................. 10

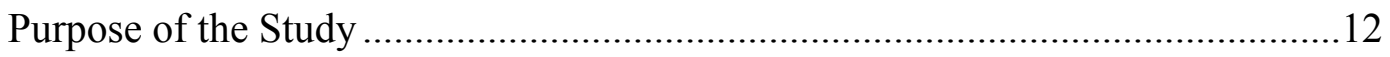

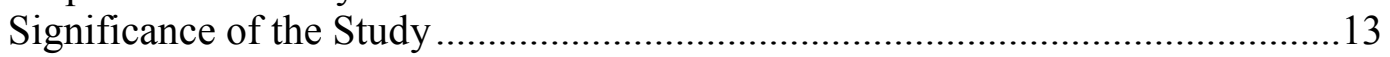

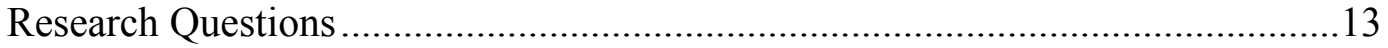

Theoretical Framework ...............................................................................

Operational Definitions of Terminology ........................................................

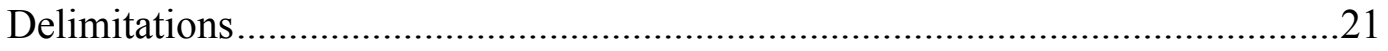

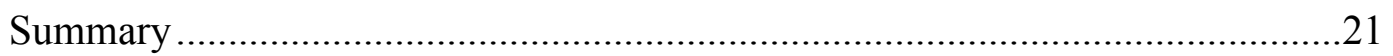

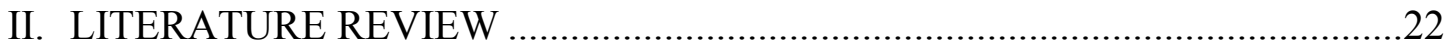

Course Scheduling: Trends and Their Influences .............................................22

Community College Decision-Making Processes ................................................24

Inattention to Longitudinal Methodology and Thoughtful Assessment ................25

Ebbinghaus and the Spacing Effect ..............................................................28

Deficient-Processing Mechanism and Encoding-Variability Theory .....................31

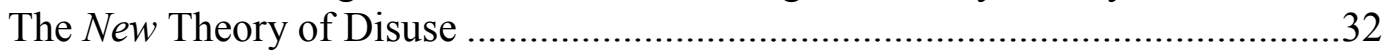

Learning-Style and Developmental-Theory Considerations ...................................33

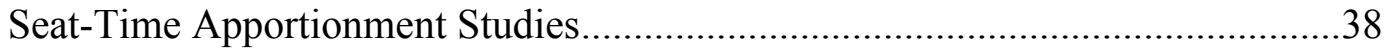

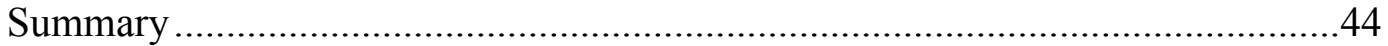

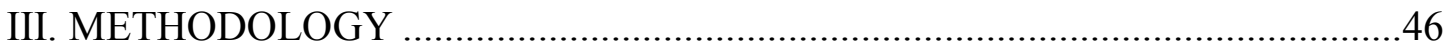

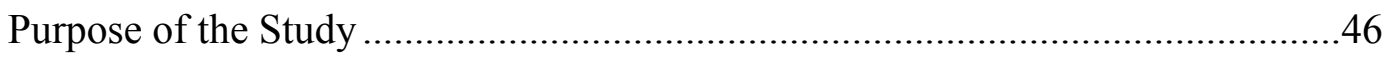

Research Questions and Hypotheses .................................................................46

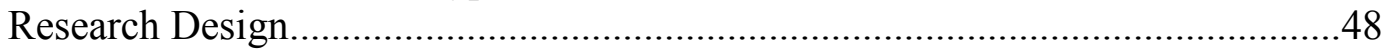

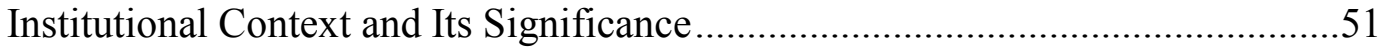

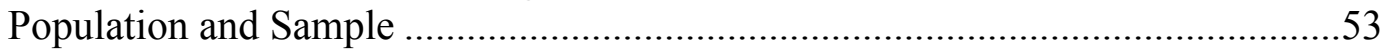

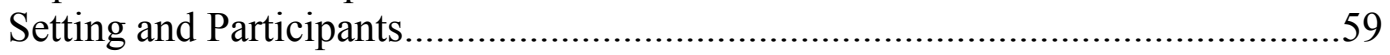

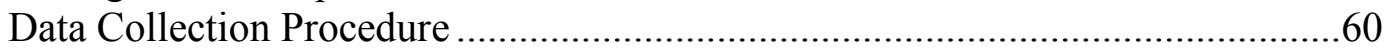

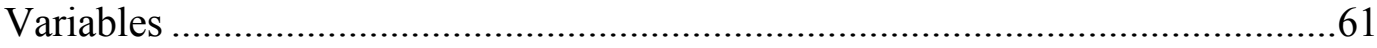

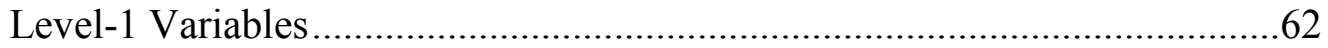

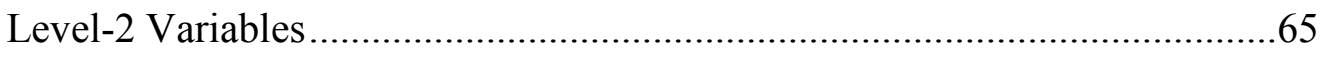

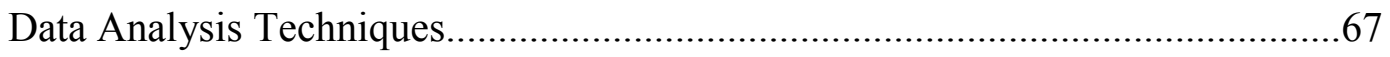

General Overview and Rationale ..................................................................67

Survival Analysis Constructs and Considerations .........................................69 
Preparation of Data Set for Discrete-Time Survival Analysis (DTSA) ..........71

DTSA Considerations for Research Question 3 ...........................................8 80

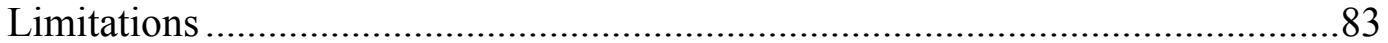

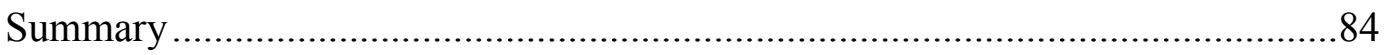

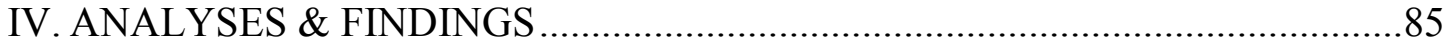

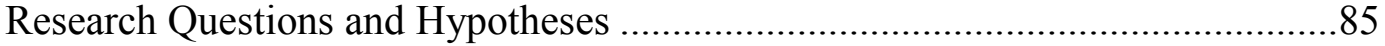

Demographic Attributes of Sample and Descriptive Statistics............................87

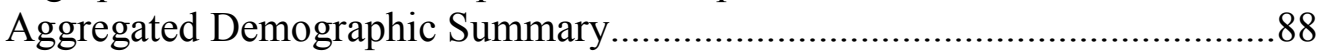

Demography Disaggregated by IV of Primary Interest (Seat Time) ..............93

Disaggregation of Academic-Related Background Covariates ......................97

Class-Section Characteristics Disaggregated by Primary IV ........................100

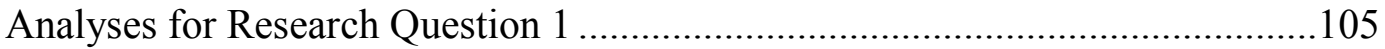

Preliminary Cross-Tabulation Analyses ..................................................105

Construction and Testing of Multilevel Logistic-Regression Models ...........106

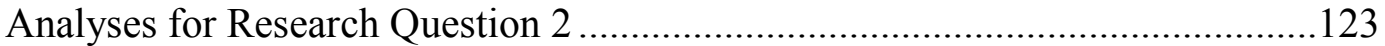

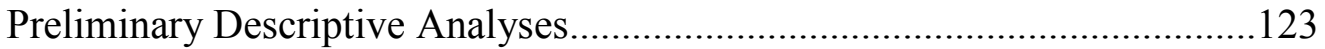

Construction of DTSA Life Table ...........................................................126

Statistical Tests for Group Differences .................................................136

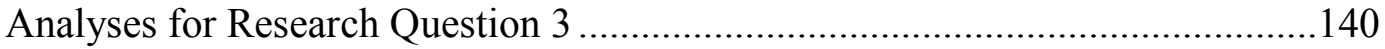

Descriptive Summaries of Cohort's Longitudinal Case Histories .................140

Construction of DTSA Life Table ..........................................................145

Results of Life-Table Differences Hypothesis Test.....................................151

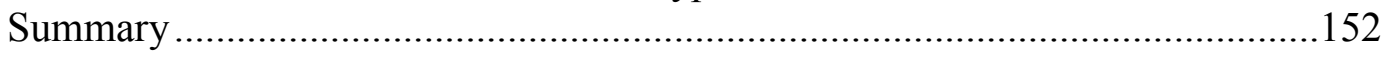

V. SUMMARY, CONCLUSIONS, \& RECOMMENDATIONS ...........................153

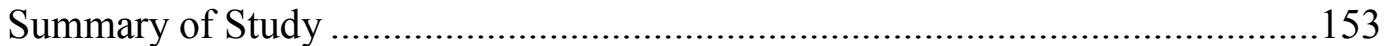

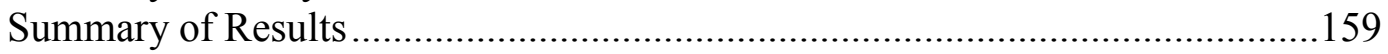

Some Unanticipated Longitudinal Limitations.............................................169

Some Very Practical Course-Scheduling Recommendations ..........................171

Recommendations for Institutions and Practitioners .....................................180

Recommendations for Researchers ..............................................................188

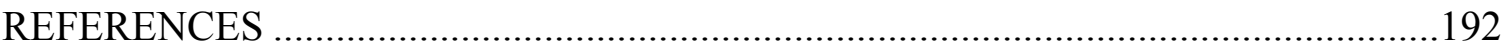

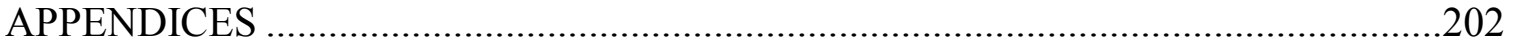

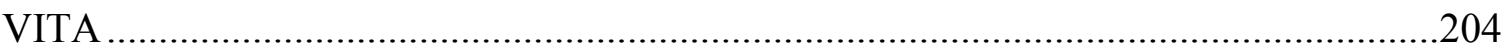




\section{LIST OF TABLES}

TABLE

PAGE

1. Fall-2001 Session Dates, Deadlines, and Durations ............................................ 72

2. Frequency Table of MAT0024 Student Cohort Demography ..............................89

3. MAT0024 Cohort Demographics Disaggregated by Seat-Time Level .................94

4. Homogeneity-of-Proportion Tests for Student Demographics by Seat Time........95

5. Homogeneity-of-Proportion Tests for Student Demographics * Seat Time..........97

6. Frequency Distribution of Academic-Related Covariates ................................99

7. Characteristics of Fall-2001 MAT0024 Class Sections .....................................101

8. Cross-Tabulation of Seat-Time Level Versus Course Success............................105

9. Cross-Tabulation of Class Location Versus Course Success ............................106

10. Intercept-Only Single-Level (GLM) Model ................................................. 109

11. Single-Level (GLM) Model with Seat-Time as Sole IV ................................110

12. Two-Level (GLMM) Null (Intercepts-Only) Model ......................................111

13. Two-Level (GLMM) Model with Seat-Time as Sole IV .................................113

14. Two-Level Model with Seat-Time and Demographic Covariates ......................115

15. Two-Level Model with Seat-Time and Academic Covariates ...........................118

16. Two-Level Model with Seat-Time and Level-2 Covariates .............................119

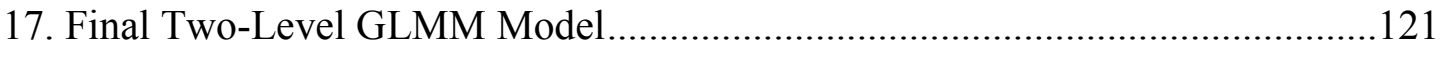

18. Cross-Tabulation of W \& non-W Grades by Session \& Seat Time .....................124

19. Life Table for Research Question 2 - Short Seat Time ...................................127

20. Life Table for Research Question 2 - Medium Seat Time ...............................128 
21. Life Table for Research Question 2 - Long Seat Time

22. Results of Actuarial-Method Hypothesis Test for Research Question 2

23. Results of Kaplan-Meier Method Pairwise Comparisons.

24. Cross-Tabulation by Seat-Time Level of Subsequent Course Attempt.

25. Number of Course Attempts Within Longitudinal Tracking Period

26. Disaggregated Frequency Distribution of GEMC Course Successes .144

27. Survival-Event Frequencies by Consecutive Time Period .146

28. Life Table for Research Question 3 - Short Seat Time

29. Life Table for Research Question 3 - Medium Seat Time 148

30. Life Table for Research Question 3 - Long Seat Time

31. Q3 - Wilcoxon (Gehan) Test of Pairwise Life Table Comparisons. 152 


\section{LIST OF FIGURES}

FIGURE

PAGE

1. Graph of Q2's Estimated Survivor Functions (weekly partitioning)..................133

2. Graph of Q2's Estimated Non-Survivor (Cumulative-Hazard) Functions ..........135

3. Graph of Q2's Estimated Survivor Functions (un-partitioned) .........................136

4. Graph of Q3's Estimated Survivor Functions (half-semester partitioning).........150

5. Graph of Q3's Estimated Non-Survivor (Cumulative-Hazard) Functions ..........151 


\section{CHAPTER I}

\section{INTRODUCTION TO THE STUDY}

This chapter begins with a restatement of this study's title, followed by a discussion of the problem's background and its significance. After that, the remainder of Chapter 1 presents, in succession, the following: a statement of the problem; the study's purpose, significance, and research questions; and, lastly, the study's theoretical framework, operational definitions of its terminology, and its delimitations.

\section{Title}

A Retrospective-Longitudinal Examination of the Relationship between Apportionment of Seat Time in Community-College Algebra Courses and Student Academic Performance

\section{Background to the Problem}

During the past decade or so, there has been a dramatic increase by postsecondary institutions in providing academic programs and course offerings in a multitude of formats and venues (Biemiller, 2009; Daniel, 2000; Kucsera \& Zimmaro, 2010; Lang, 2009; Mangan, 2008; Tallent-Runnels, Thomas, \& Lan, 2006). Strategies pertaining to reapportionment of course-delivery seat time have been a major facet of these institutional initiatives. From executive-track programs to weekend offerings to hybrid web-assisted and fully online courses/programs to compressed/intensive mini-terms, many colleges continue to devote an increasing proportion of their resources to recruitment and accommodation of non-traditional students. In large measure, the basis for these recruitment efforts has been two-fold. 
First, there has been the influence of consumer demand, market forces, and earmarked government funding. Peterson and Dill (1999) posited that a true understanding of contemporary higher-education leadership at the institutional level must begin with recognizing the sharp distinction between the major catalyst for change during the latter half of the twentieth century—namely, federal, state, and institutional initiatives—with the dominant change-agent that looms ahead in the twenty-first century: specifically, competitive market forces and the larger societal environment.

Historically, the traditional approach to planning in postsecondary education typically had been to gather information about environmental changes/trends and then develop a modicum of institutional strategies to respond. The implicit assumption of institutions had been that their competition was limited to other established institutions in a loosely defined (but certainly identifiable) system of higher and postsecondary education. Peterson and Dill (1999, p. 508) argue that this is antiquated thinking, since societal and market forces are transforming the fundamental nature of postsecondary education into a "postsecondary knowledge system or industry," one that delivers instruction, knowledge, and information in many forms over "a vast and flexible learning network" that transcends traditional notions of "institution" and "system".

Among the participants who pervasively influence this more amorphous and multi-faceted knowledge system are, to name a few, telecommunications companies, proprietary institutions, corporate-training providers, software manufacturers, and, of course, the Internet. Hence, in this hyper-competitive environment, it is not surprising that a common marketing device used in most efforts to recruit nontraditional students is 
publicity of decreased student "seat time" or, at least, a reapportionment of seat time in a way that minimizes the number of face-to-face course meetings.

Second, many colleges have been facing an unfamiliar paradox: burgeoning enrollments yet dwindling capital-improvement fund balances (Biemiller, 2009; Daniel, 2000; Lang, 2009; Mangan, 2008; Okpala, Hopson, \& Okpala, 2011). Such budgetary constraints in recent years have induced many institutions to reconfigure their course master schedule, in an effort to more efficiently use their existing classroom space while simultaneously decreasing facility-related costs.

One prominent illustration of this is the trend toward block scheduling models compressed into fewer days per week; for example, various colleges — as well as other public sector agencies — have been experimenting with 4-day workweeks (Mangan, 2008). In fact, today there are many academic disciplines at various institutions in which all classes meet no more than once per week in large time blocks of three or more hours per meeting. When other disciplines within the same institution do not follow suit (e.g., those that offer required general-education courses that are more grounded in the development of higher-order skills and more advanced abstract reasoning processes, of which some are part of a multi-semester sequence of courses), conflicts in the design of the institution's master schedule tend to arise. Sometimes, these conflicts have adverse consequences upon enrollment management, instructor staffing, retention rates, students' financial aid, and academic quality.

\section{Significance of the Problem}

While these aforementioned potential detriments are worthy of concern across all higher-education strata, they are especially severe at the community college level. For 
community colleges, there are not only national-level aspects to this problem that are virtually ubiquitous, but also there are additional state-level dimensions and institutional decision-making implications that further exacerbate its gravity.

\section{National Level}

According to the Pew Research Center (2009), nearly one-third of 18-to-24 yearold U.S. undergraduates are enrolled in community colleges, which equates to approximately $12 \%$ of the nation's entire 18 -to- 24 year-old population. The American Association of Community Colleges (Mullin \& Phillippe, 2011), in collaboration with the National Students Clearinghouse, reported that out of the 5.83 million total college student recipients of Pell grants in the first quarter of academic year 2011-2012, just over two million of these students are enrolled at public 2 -year colleges. This reflects a $17 \%$ increase in the number of community-college student Pell-grant awardees as compared to the previous year. In monetary terms, public 2-year college students received $\$ 3.6$ billion, or $32 \%$, of the $\$ 11.6$ billion awarded in Pell Grants during the fall term of 2011.

Amid these large increases in enrollment and federal financial aid, national community-college graduation rates, on average, remain distressingly low. According to the nonprofit organization Complete College America (2010), "Current [community college] completion rates for full time students average $25 \%$ at the end of three years and part time graduation rates rarely exceed $10 \% "$ (p.1 of Appendix E). In the case of Florida's community college system, these rates are even lower: $17.9 \%$ and $4.2 \%$, respectively (Complete College America, 2011).

Historically, community college enrollment trends are negatively correlated with the nation's economic health; that is to say, community colleges experience high 
increases in enrollment during times of recession and high unemployment rates. So, with almost one out of every eight of the nation's young adult population being enrolled in a 2-year college and with billions in public monies expended in subsidizing their education, the endemically high rates of community college student attrition is a concern with broad societal impact, socioeconomically and otherwise.

For example, consider the large role 2-year public institutions play in furthering opportunity for historically under-represented groups, dating back to the passage of the Civil Rights Act of 1964. As Mercer and Stedman (2008) attest, the nation's colleges and universities that are classified as minority-serving institutions (MSIs) are disproportionally community colleges. "[MSIs] play a special role in the education of minority and low-income students. This group of institutions annually receives a significant amount of federal funding [e.g., $\$ 895$ million in 2005] to pursue their educational mission and educate minority students" (p. 40).

Consequently, as the United States' population continues to shift toward a minority-becoming-majority demography and as large sums of public monies continue to be invested in the pursuit of making the American Dream realizable for all, high dropout rates within 2-year colleges pose a serious detriment to the country's socioeconomic future. Furthermore, the general public can be expected to vociferously decry any large appropriation of social-program funds that fails to yield commensurately large results, especially during a period of widespread economic strife. Failing to get results also serves to lend support to those who politically and philosophically oppose diversity initiatives based on Fourteenth Amendment (equal protection) grounds (Brown II, Butler, \& Donahoo, 2004). 
For these reasons, as the pressure on community college leaders to find ways to improve graduation rates continues to mount, course-delivery and course-scheduling paradigms are one institutionally controlled variable that may be part of the solution. Recent scholarly research supporting this contention has been embraced by a number of politically-influential nonpartisan foundations. For example, in a report requested by the White House Working Group for the President's Summit on Community Colleges (Complete College America, 2010), the research-based perspective of Rosenbaum, Redline, and Stephan (2007) was extensively referenced:

Community colleges allow students to explore broadly in liberal arts and to progress at their own pace, assuming that students have clear plans and can assess which classes will fulfill those plans. When students have information problems, community colleges respond by piling on more information: more brochures, more catalog pages, and more meetings. For students unfamiliar with college and inexperienced at handling large amounts of information, information overload can result. Moreover, in providing many options, community colleges also create complex pathways, dead ends, and few indications about which choices efficiently lead to concrete goals. (Rosenbaum et al., p. 51)

\section{State Level}

In the case of Florida's public colleges and universities, there is another dimension of this issue that is important to consider. During the past thirty years, statebased financial-aid support has continued to decline. Most states have reduced the taxpayer's share of the responsibility by markedly increasing tuition rates and relegating much more of the financial responsibilities to students and their families (Chen \& St. 
John, 2011). Within the past 15 years, two of America's most populous states, first Florida and more recently Texas, have enacted more austere policies which impose strict enrollment-eligibility limits and significant financial penalties upon students who have to repeat a given course more than once.

More than a decade ago, the Florida legislature instituted a policy commonly known as the three-course-attempt rule, whereby students are limited to three attempts per course. An attempt is defined as remaining in the course past the tuition-refund deadline, which typically occurs within a week after the class commences (Florida State Board of Community Colleges, 1998; State Board of Education, 2004). When a third attempt is required, students are not permitted to withdraw or audit (i.e., a letter grade must be awarded), and resident students must pay the non-resident tuition rate, which can be in excess of triple the resident rate. For several years, Florida's three-course-attempt rule was a national anomaly, until the state of Texas implemented in 2006 what it dubbed its three-peat rule, a piece of legislation very similar to Florida's (Texas Higher Education Coordinating Board, 2005).

When enacted in the fall of 1997, Florida's three-attempt rule was a dramatic change for its community college students and employees. For inexperienced undergraduate students who have not, for the most part, had a history of academic success and are prone to making imprudent course-scheduling choices, the ramifications of the three-attempt rule upon their aspirations to earn a degree can be ruinous.

For example, as an academic administrator at a large community college, the author of this dissertation was involved in the scheduling, assignment of instructors, and extensive promotion of special sections of algebra courses geared to help at-risk students 
who had failed to pass during one or more previous course attempts. These special course sections afforded students the opportunity to have additional class contact time with some of the most pedagogically-skilled faculty, along with other "amenities," yet without the imposition of any additional tuition or fees. Despite widespread publicity and studentoutreach efforts, a large proportion of these specially-arranged course sections ultimately had to be cancelled because many of these at-risk, recidivist students, when left to their own devices, wanted to enroll in course sections that required the fewest number of class meetings with no more than the state-mandated minimum amount of seat time.

For community college instructors who are passionate about helping students, it is demoralizing to witness their respective institution's open-door policy turned into a revolving door characterized by rising student-attrition rates. In light of the bleak financial state of many public colleges and given the relationship that exists between poor decision-making in course selection and low academic success rates, Hagedorn, Maxwell, Cypers, Moon, and Lester (2007, p. 480) encourage “ “... actions to curtail unnecessary course shopping by assisting students to make wise choices the first time... [for] the numbers of dropped courses may signify the need for change." The research of Rosenbaum et al. (2007) echoes this recommendation:

Although students are assumed to be capable of making informed choices, of knowing their abilities and preferences, of understanding the full range of college and career alternatives, and of weighing the costs and benefits associated with different college programs, our analyses show that many students have great difficulty with such choices... [and they] have poor information about remedial 
courses, course requirements, realistic timetables, degree options, and job payoffs. (p.51)

In light of this type of student demography, it is not surprising why, for at-risk students who are often deficient decision-makers, having to repeat a given course is such

a common occurrence. Consequently, it is reasonable to conjecture that the sorts of legislatively-mandated limits and penalties imposed upon Florida and Texas undergraduates who must re-attempt a course further exacerbate these states' alreadyendemic attrition rates. It should be noted that the author of this dissertation was hardpressed to find any peer-reviewed study pertaining to either Florida's or Texas's threeattempts-per-course rule, nor even any that have incorporated the number of course attempts in a multivariate analysis. This being the case, the potential effect of this threeattempt rule and individual students' numbers of course attempts has been worthy of (and, arguably, has been overdue for) inclusion in a study of the relationship between seat-time apportionment and success/persistence in community-college course offerings.

\section{Institutional Decision-Making Implications}

As their institutions continue to proliferate new course-design and seat-time formats largely designed to increase market share, most community college students continue to have virtually no constraints upon their enrollment decisions. Paradoxically, higher education has begun, over the past several years, to experience an accelerating shift toward greater accountability.

Institutional and programmatic reaccreditation processes have become more rigorous, requiring colleges to demonstrate and quantify student achievement and institutional quality enhancements (Morest \& Jenkins, 2007). Federal and state 
government funding sources (e.g., the massive grant program contained within the Carl D. Perkins Vocational and Technical Education Act) have undergone significant restructuring — and even continue, at present, to undergo further alterations — in their application (and renewal/retention) processes; most notably, their accountability mechanisms (Stasz \& Bodilly, 2004).

Meanwhile, as community colleges continue to apply considerable resources to enrollment management strategies, this era of increased accountability throughout the K20 educational system has given some educational leaders pause to re-assess the institutional decision-making processes pertaining to matters like course design/delivery and seat-time apportionment. In a September 2005 address on the campus of University of North Carolina at Charlotte, U.S. Secretary of Education Spellings announced the formation of the Secretary of Education's Commission on the Future of Higher Education:

It is time to examine how we can get the most out of our national investment in higher education. We have a responsibility to make sure our higher education system continues to meet our nation's needs for an educated and competitive workforce in the 21st century. (U.S. Department of Education, 2005, para. 2)

\section{Statement of the Problem}

Largely driven by a desire to increase market-share, afford non-traditional students course options that better fit their scheduling desires, optimize the usage of finite facility capacity, and contain costs during these economically turbulent times, many open-door 2-year institutions continue to devote an increasing portion of their enrollment-management resources toward the expansion of their offerings of course- 
delivery formats and seat-time apportionment configurations. However, based upon this dissertation author's literature review and his varied professional experiences, it appears that very few community colleges, if any, have given more than fleeting consideration to the effects these course-delivery approaches may have on student learning, course success, enrollment persistence, and time to program completion.

Furthermore, most 2-year colleges lack the institutional-research capability and research-methodology awareness to study such matters in a rigorous manner (Achieving the Dream Community Colleges Count, 2005-2010), especially with the aid of an assortment of powerful multilevel-longitudinal analysis techniques developed over the past two decades. To date, such quantitative methods have been largely absent in educational research, despite their rapidly widening use among researchers in a wide variety of academic disciplines during the past two decades (Willett \& Singer, 2004).

Therefore, on the basis of both the rationale cited thus far and the broad range of additional reasons that shall be elaborated upon later in this introductory chapter and subsequent chapters, there is a compelling need for a comprehensive study (i.e., one that was multilevel and longitudinal) of the impact of seat-time apportionment in communitycollege level mathematics, an academic discipline that is one of the two largest generaleducation content areas, with often the highest attrition rate in undergraduate education and customarily the one with the highest percentage of remedial-level enrollees.

Among the 15 or so mathematics courses available at most community colleges, the greatest research need has been an examination of the effects of seat-time apportionment in the sequence of core algebra courses, which is commonly comprised of pre-algebra, elementary algebra, intermediate algebra, and college algebra. Virtually 
omnipresent throughout American community college curricula, these four courses have, by far, the highest enrollment volume and are commonly viewed as gateway courses due to their completion being a prerequisite to entry into most any degree program.

This researcher formulated a tripartite approach to addressing the problem. First, the study sought to ascertain the nature of the relationship, if any, between the apportionment of seat time and student learning gains in algebra courses. Second, the research created a multivariate model (e.g., a survival-analysis model) to assess whether there was a significant relationship between seat-time apportionment and the time when students withdraw from a course. Third, in a retrospective longitudinal manner, an examination was conducted of whether academic performance in subsequent algebra courses was related to the seat-time apportionment of previous prerequisite courses.

\section{Purpose of the Study}

The purpose of this retrospective-longitudinal study was to investigate whether scheduling courses in large time blocks (e.g., three or more hours once per week) is significantly related to the academic performance of community college students who enrolled in one or more remedial-level mathematics courses offered in this format, as compared to the academic performance of students who enrolled in courses that met more frequently and in medium-length time blocks (of 75 minutes twice per week) or shorterlength time blocks (of 50 minutes thrice per week).

A longitudinal research design and multilevel analysis techniques were essential components of this study because, unlike cross-sectional multivariate studies, this author's research methodology: (a) enabled the construction of multilevel models that are better equipped to account for and explain variations between individual students, 
between various courses, and between different seat-time apportionment configurations; (b) more appropriately addressed the effects of missing data and unobserved survival events (e.g., persisting in a course or program versus withdrawing); and (c) analyzed the seat-time apportionment effects, if any, on students' rates of progression and success in these compulsory, sequential algebra courses.

\section{Significance of the Study}

If the study demonstrated evidence of a significant relationship between course success and seat-time apportionment, these findings would have implications for better informing college administrators and faculty who construct course schedules each semester, as well as academic counselors who assist and advise students during the registration process. Also, the ex post facto longitudinal research design and analysis methodology that was utilized may serve to broaden the data-analysis perspective of institutional-research professionals who are often charged with compiling and analyzing retention/attrition data yet seldom employ these types of increasingly popular and computationally practicable statistical tools.

\section{Research Questions}

The central research question that this study addresses is: Is there a relationship between seat-time apportionment in community-college algebra courses and course success, course enrollment persistence, and time to complete general-education level mathematics coursework?

The exploration of this overarching research question was subdivided into three component questions, in which student success was operationalized based upon the student's final letter grade earned in a given course: 
1. In community-college developmental-level algebra courses, is there a difference in student success for students enrolled in classes that meet one day per week in longer-duration class meetings versus for those enrolled in medium-duration (twice weekly) class meetings versus for those enrolled in shorter-duration (thrice weekly) class meetings?

2. In community-college developmental-level algebra courses, is discrete survival time, as measured by date of withdrawal from the course, related to the number of class meetings per week and the duration of each meeting?

3. Across the sequence of community-college algebra courses, is there a longitudinal difference in the time to complete at least one general-education college-level mathematics courses between students who satisfied the prerequisite algebra course requirements by completing one or more algebra courses scheduled in a one-day-per-week, longer-duration format and students whose prerequisite algebra course requirements were entirely fulfilled in courses that had medium-duration (twice weekly) or shorter-duration (thrice weekly) class meetings?

\section{Theoretical Framework}

The learning-theory constructs associated with reapportionment of seat time for course delivery pre-dates the field of experimental psychology. Ebbinghaus (1885/1964) pioneered the notion of quantifying the dependence between the formation (and level of inculcation) of memories of learned content that is completely new to the individual and the duration/pace of (and the time gaps between) each study session. In today's scholarly literature, the term used for this cognitive phenomenon is the spacing effect. 
Ebbinghaus's experimental findings lead him to conclude that “... with any considerable number of repetitions a suitable distribution of them over a space of time is decidedly more advantageous than the massing of them at a single time" (Ebbinghaus 1885/1964, p. 89).

Over the 125-year span since Ebbinghaus's groundbreaking study of the spacing effect, a wide array of subsequent research has corroborated his conclusions and expanded their applicability (Cepeda, Vul, Rohrer, Wixted, \& Pashler, 2008; Dempster, 1988; Hilgard, 1964; Kornell \& Bjork, 2008). Nonetheless, despite the voluminous amount of scientific examination of the spacing effect over many decades, many scholars have pointed out the lack of experimental inquiry into the spacing effects role in general classroom-type learning and, in particular, mathematical-skill development (Cepeda, Vul, Rohrer, Wixted, \& Pashler, 2008; Dempster, 1988; Rohrer, 2009; Rohrer \& Taylor, 2006). This gap in the literature supported the need for a longitudinal-type study of the spacing effect in the context of a community-college mathematics classroom.

Among the most prominent cognitive-based justifications for the spacing effect phenomenon are the deficient-processing mechanism and encoding-variability theory (Toppino \& Bloom, 2002). The former posits that the quantity of the information processed and the quality of the memory encoded is greater when the learning experience is scheduled in a way that allows for properly spaced reinforcement and processing opportunities; the latter theory is predicated on the notion that multiple, less comprehensive learning sessions — as opposed to fewer, massed sessions—aid retention by giving the learner opportunities to mentally encode the subject matter in a variety of ways. Bower (1972), Glenberg (1979), and Melton (1970) are among those whose work 
has independently supported encoding-variability theory as an explanation for the spacing effect.

More recently, other theories have been conjectured; for example, Bjork and Bjork's (1992) new theory of disuse. With regard to this dissertation study's research problem, what is most notable about this complex, multifaceted theory is the distinction it draws between the traits of the spacing effect in learning something new for the first time in contrast to relearning something experienced sometime in the past. Although relearning is often the case with community-college algebra students, the demographic diversity of the students is such that - in most any given class with most any given mathematical concept—some students will be experiencing first-time learning while others are relearning. Being able to disaggregate the measurement of performance in a learning versus a relearning situation would likely be unwieldy in a cross-sectional research design. In contrast, a retrospective-longitudinal design would better facilitate the use of each student's prerequisite coursework and recency thereof as an independent variable that, at least in part, distinguishes original learning from relearning.

In summary, there an eclectic array of constructs that comprise the theoretical framework intertwined with what this dissertation has termed seat-time apportionment. In very general terms, these constructs fall into three overlapping categories: informationprocessing and developmental theories; institutional environment and community-college student attrition rates and related theoretical models; and classroom-environment research. In Chapter 2 of this dissertation, all three of these aspects of seat-time apportionment shall be explored further. 


\section{Operational Definitions of Terminology}

\section{Academic Performance}

A dichotomous delineation between the operationally defined terms "course success" and "course failure."

\section{Course Attempt}

Enrollment in the course past the refund deadline, which typically occurs after one week of instruction; for example, a student who withdraws from a course after 3 weeks or changes his/her enrollment status from credit-seeking to audit has used one course attempt.

\section{Course Delivery Type}

A distinction between courses that are fully online, blended/hybrid (e.g., those that reduce the total amount of face-to-face contact time by $25-50 \%$ ), and traditional lecture style (i.e., those without a reduction in face-to-face instruction time).

\section{Course Failure}

Applies to a student who registered as credit-seeking and receives a final course grade that is something other than a " $\mathrm{C}$ " or higher, including a withdrawal after the refund deadline.

\section{Course Passing Rate}

The proportion of students in a given course section that obtained a grade of "C" or higher. 


\section{Course Persistence}

A ratio-level measure reflecting the percentage of a given course a student completed without withdrawing or being withdrawn (for non-attendance) by the instructor.

\section{Course Success}

A final course grade of " $C$ " or higher.

\section{Enrollment Status}

A distinction between a full-time enrollee (i.e., a student enrolled in at least 12 semester hours of credit in a given semester) and a part-time enrollee (i.e., a student enrolled in fewer than 12 semester hours of credit in a given semester).

\section{Instructor Status}

A "full-time" status applies to any tenure, tenure-track, or full-time temporary faculty member, whereas a "part-time" status pertains to instructors who are hired term by term, course by course (also known as adjunct instructors). Some part-time instructors may be full-time non-instructional personnel within the institution; for example, administrators or learning resource center support staff. Since the percentage of developmental-level algebra coursework taught by part-time faculty is typically high, instructor status was included as a control variable due to perceptions held by some fulltime faculty and administrators of grade inflation by part-time faculty. These perceptions are often attributed to part-time faculty being, on average, less well versed in the discipline's academic expectations and being employed on a term-by-term, course-bycourse basis without any contractual/union protection. 


\section{GPA}

Grade point average, which may be treated in various ways, such as overall weighted GPA at the institution (but without consideration of the institution's gradeforgiveness practices) or, for example, overall weighted GPA in mathematics courses only.

\section{Meeting Number}

The total number of face-to-face class meetings for the given course in the given semester (based upon the days of the week the class was schedule to meet, the class's session start/end dates, the institution's instructional calendar, and its final-exam schedule).

\section{Placement Test Score}

An exam score used to determine a student's initial math-course placement. As warranted, these scores are rescaled in terms of a measure of relative standing; for example, rescaling in the form of a percentile to enable comparisons between those whose course placement was based upon an institutionally-administered placement exam—-such as the College Placement Test (CPT) or the Postsecondary Education Readiness Test (PERT) — and those whose course placement was based upon a college aptitude test (e.g., the SAT or ACT).

\section{Prerequisite Recency}

The number of semesters that elapsed between beginning a given course and completing the prerequisite course. 


\section{Seat Time}

Initially defined as the number of minutes per class meeting (i.e., the duration of each face-to-face class session). However, it should be noted that, during the data collection and analysis process, it was determined that a more suitable operationalization of seat time was the number of class meeting days per week (i.e., the frequency/spacing of class meetings). This alteration is elaborated upon in Chapter 4 (pp. 102-104).

\section{Session Length}

The length of time a course lasted, which may be quantified in terms of calendar duration measured in weeks or days; for example, a 16-week Session I Fall-2001 class, in contrast to an 8-week Session IV class within the same semester.

\section{Student Success}

Operationally defined in the same way as "course success."

\section{Survival Time}

Defined in two ways: (a) for the second research question, the number of days enrolled in the MAT0024 class until academic semesters elapsed between the start of the class's session starting date and the recorded date when the student was assigned a withdrawal ("W") grade in the course; and (b) for the third research question, the number of semesters elapsed between the end of the student's Fall-2001 MAT0024 course attempt and the successful completion of a general-education-level mathematics courses.

\section{Time of Day}

A coding of whether the class meetings were scheduled during the day (i.e., ending before $5 \mathrm{pm}$ on weekdays), weeknights, or weekends. 


\section{Withdrawal Time}

A coding of the date (or stage of the academic term) when a student withdrew or was withdrawn (for non-attendance) from a course.

\section{Years since High School}

The number of years between commencing enrollment in the institution and either high school graduation or GED equivalency completion.

\section{Delimitations}

This study limits its consideration to students in a specific subset of algebrarelated courses within one academic discipline at one large multi-campus community college, located in an urban area of Florida that is ethnically, racially, and socioeconomically diverse. Every one of the mathematics courses considered was either entirely comprised of algebra-type curricular content or required successful prior completion of algebra coursework as an enrollment prerequisite. Because of its reliance upon a single-institution data set, this study was unable to consider institutional-context covariates, which recent research (Chen, 2012; Titus, 2004) has shown to be significant when investigating postsecondary student persistence.

\section{Summary}

This introductory chapter provided the statement of the problem and a presentation of its background and significance. The study's purpose, significance, research questions, and delimitations were also addressed, which were enhanced by a cursory discussion of the study's theoretical framework and operational definitions of terminology. The next chapter provides a literature review that elaborates upon the theoretical framework outlined in Chapter 1. 


\section{CHAPTER II}

\section{LITERATURE REVIEW}

This chapter is comprised of a five-part review of the scholarly literature relevant to the matters of course scheduling and seat-time apportionment. First, the chapter begins with a review of the predominant trends and influences related to postsecondary-level course scheduling. Second, the issue of course scheduling is elaborated upon within the broader context of community college decision-making processes. Among the matters discussed in this regard is the common problem of institutional research departments' deficiencies in assessment practices and their inattention to more recent advances in longitudinal-analysis methodologies. Then, thirdly, the chapter summarizes a variety of cognitive learning theories and psychometric concepts, including the spacing effect, the deficient-processing mechanism and encoding-variability theory, and the new theory of disuse. Fourth, learning style and developmental theory considerations are addressed. And, finally, the chapter closes with a discussion of past seat-time apportionment studies that pertained to community-college mathematics courses.

\section{Course Scheduling: Trends and Their Influences}

Over a period of two years, the author of this dissertation had the opportunity as a department-level academic administrator to fundamentally redesign a large course schedule on two community college campuses, amounting to approximately 225 course sections per major semester. He experienced first-hand many of the challenges and implications of doing so, including those related to student learning, instructor staffing, and enrollment management. Additionally, the college at which this author is employed recently began piloting a new mathematics course-delivery approach that stands in 
marked contrast to past trends at public 2-year colleges, but is comparable in some respects to the intensive seat-time formats prevalent among proprietary institutions and technical schools (Bugay, 2000; Wilson, 2010): namely, offering remedial and generaleducation level courses in a 4-day-per-week immersion-type format for eight weeks (with 75-minute class meetings), which would enable students to complete two mathematics courses in the span of one 16-week semester.

In recent years, there has been a rampant proliferation in new course-deliverysystem types and seat-time-apportionment arrangements at postsecondary institutions across the nation (Biemiller, 2009; Daniel, 2000; Kucsera \& Zimmaro, 2010; Lang, 2009; Mangan, 2008; Tallent-Runnels et al., 2006). This trend has been, for the most part, motivated by three overarching objectives: the desire to increase market-share as competition from advertising-savvy proprietary colleges and technical schools has continued to intensify; the necessity of maximizing the use of limited classroom-space capacity, especially in some states, such as Florida, where severe cuts in capitalimprovement funding have been further compounded by record amounts of gubernatorial line-item vetoes (Sanders, 2011); and the general urgent need to control costs in the midst of skyrocketing insurance costs, a severe national recession, and the looming instability of a global financial crisis.

Unfortunately, as evidenced by this dissertation author's literature review and his varied professional experiences, what seems largely absent from many postsecondary institutions' course-scheduling decision-making processes is serious contemplation and investigation of the potentially deleterious role these marketplace-driven course-delivery offerings may play in affecting the rates of student learning, course success, enrollment 
persistence, and time to program completion. This managerial deficiency is particularly acute at the open-door, public community college level, where the institutional-research infrastructure and research-methodology awareness needed to examine these matters accurately are often lacking (Achieving the Dream Community Colleges Count, 20052010).

\section{Community College Decision-Making Processes}

Romero, Purdy, Rodriquez, and Purdy (2005) are among those who lament that relatively little study of community college decision-making processes has been performed. They contend that the present need for such research is great in light of the current rapidity of intra-organizational change occurring within America's community colleges, at most every level conceivable. Among these changes are high administrative turnover rates (often, with little training of new leaders), technology uses, student demography/enrollment shifts, and funding/policy/mission-related challenges. In recent years, several national initiatives have been launched to increase awareness of what is often termed data-driven decision-making. Among them is the Achieving the Dream: Community Colleges Count initiative, which Morest and Jenkins (2007) characterized as follows:

Aided by a data facilitator and a coach, colleges participating in Achieving the Dream collect longitudinal data on their students... analyze... and disaggregate the findings to determine if there are gaps in achievement among particular student groups.... Colleges can then develop strategies to improve student progress based on a clear diagnosis of the challenges that are present. (p. 2) 


\section{Inattention to Longitudinal Methodology and Thoughtful Assessment}

This type of longitudinal investigation involving aggregating and disaggregating is sometimes referred to as multilevel longitudinal analysis. It is a powerful collection of quantitative methodologies about which more will be written in the next (methodology) chapter of this dissertation. Two points, however, are worthy of mention here.

First, whereas many fields of scholarly inquiry_ranging from economics to sociology, from agriculture to psychology to biomedicine-have witnessed an exponential increase in the use of such longitudinal methodology over the past two decades, education research has not been among them. In fact, Singer and Willett (2004) claim that in published educational research, from 1982 to 2002, there was an eight percent decrease in the use of longitudinal methods. It is their contention that "Education is falling behind... [in various areas of] quantitative research.... Given the power of modern longitudinal methods to address research questions about change and event occurrence, this as a serious problem that needs addressing” (Notes portion of PowerPoint Slide \#5). The effect of seat-time apportionment on student achievement is one example among countless many of an educational research topic that is ripe for longitudinal scrutiny.

Second, the previous point begs the question: Why have the great advances in longitudinal quantitative methods over the past several decades not been embraced by the educational research community? Part of the answer can be found in national initiatives like Achieving the Dream (ATD). ATD and other comparable enterprises are, to a large extent, an effort to reform the decision-making ethos of America's community colleges, for most institutions are woefully ill-equipped - in terms of institutional-research 
department-level infrastructure, as well as trans-institutional data-analytic awareness - to neither conduct nor interpret the types of analyses that would serve to best inform college leaders when contemplating student-outcome data in matters such as seat-time apportionment (Achieving the Dream Community Colleges Count, 2005-2010). So, although many college administrators find it politically desirable to characterize themselves as data-driven decision-makers, the reality is that few have the necessary time, resources, or acumen (Romero et al., 2005).

Levin and Calcagno (2008) noted that the institutional research performed at most community colleges is not conducive to data-driven policy-making, for it largely consists of simple cross-tabulation reports mandated by federal and state governmental agencies, and also by institutional and program-specific accreditors. Most institutional research departments are “.... staffed by only a single professional with limited clerical support, and in some cases, that professional lacks training and experience in evaluation. In short, there exists little real capacity to carry out rigorous research at most community colleges" (pp. 201-202). Without such capacity, Levin and Calcagno argue, methodologically sound and systematic experimentation and evaluation of academic programs-in particular, with regard to remedial education — cannot occur.

These widespread leadership and research-infrastructure deficiencies lend credence to the perspective held by those who question whether at many colleges the course scheduling decision-making process, including seat-time apportionment, has become too grounded in market-related and budget-type concerns, along with individual faculty scheduling preferences, with, at best, a small amount of consideration given to intra-disciplinary pedagogical best-practices and methodologically-appropriate analyses 
of disaggregated student data. Questions like this give rise to others, such as: Are academic administrators paying sufficient attention to the effect their course-scheduling practices have on student achievement and, in particular, skill mastery? Are their efforts at all shortsighted, in the respect that decreasing the number of times a course has face-toface class meetings (sometimes by increasing the seat-time duration of each meeting) may increase market share but may be doing so at the expense of decreasing studentretention and program-completion rates and learning gains, especially among the at-risk students who enter 2-year colleges needing to complete remedial-level (i.e., collegepreparatory) coursework?

McClenney, McClenney, and Peterson (2007) attempted to answer questions like these in a broad context, by providing community college leaders with an institutionalplanning model for how to go about cultivating a true culture of evidence, in contrast to what some, like Morest and Jenkins (2007), describe as a culture of "institutional mythology" (pp. 3, 12). Troubled by the wide disparity between degree-completion rates at community colleges versus those at 4-year institutions, these three authors call for major transformational change at the nerve center of our community colleges, not just cosmetically tweaking around the edges. They contend that the first step toward transformational change is acquiring a deeper understanding of (and awareness of how to use) data pertaining to their students' experiences. Their belief is that this present era of increased accountability is a perfect time to surmount the general resistance toward thoughtful assessment and data-driven decision-making processes that has long pervaded the higher education community. A heightened appreciation of the potential contributions of psychometrics and experimental psychology would certainly further this aim. 


\section{Ebbinghaus and the Spacing Effect}

The psychometric constructs associated with reapportionment of seat time for course delivery can be traced to an often overlooked, yet highly influential forerunner in the history of modern experimental psychology and psychometrics: Hermann Ebbinghaus (1850-1909). Ebbinghaus (1885/1964) was the first to attempt to quantify the dependence between the formation (and degree of permanence) of memories of materials that are completely new to the individual and the conditions under which the material has been studied. The conditions he focused his attention on were primarily the duration and pace of each study session and the time gaps between sessions.

The meticulousness with which Ebbinghaus designed his memory experiments is especially notable. He was keenly aware of the many-centuries-old theory of mind that learning, from birth, is an ever-growing network of associations and that committing content to memory is a process of forming associations with past memories and experiences (Mook, 2004; Tulving, 1985). Ebbinghaus wanted to build memories from zero strength to a certain benchmark, in order to avoid the confounding variable of recollective experiences (what some scholars term retrieval cues), which obviously differ significantly from individual to individual. To achieve this, he invented his now-famous sets of nonsense syllables, which were formed from two consonants with a vowel between them. Any of these three-letter sets that corresponded to an actual word were discarded. He concluded there is a high likelihood that “... with any considerable number of repetitions a suitable distribution of them over a space of time is decidedly more advantageous than the massing of them at a single time" (Ebbinghaus 1885/1964, p. 89). 
This notion of learning being enhanced by a series of shorter, separated sessions - rather than fewer, more massed episodes - is frequently referred to in the scholarly literature as the spacing effect. Other terms for this phenomenon-albeit with some variation in definitional details - include the lag effect and the distributed-practice effect.

To this day, despite how revered Ebbinghaus remains for both his 1885 seminal volume on memory and his pioneering use of (and fearless advocacy) for experimental methods during the field of psychology's formative years (Fuchs, 1997), some reflexively and prematurely dismiss Ebbinghaus's work because he performed his experiments on only one subject: himself. He himself acknowledged this limitation but contended that his conclusions were far from idiosyncratic and, instead, were generalizable. More than a century's worth of scholarship has shown his contention to be true (Cepeda et al. 2008; Dempster, 1988; Hilgard, 1964; Kornell \& Bjork, 2008). "Indeed, the spacing effect is arguably one of the largest and most robust findings in learning research, and it appears to have few constraints" (Rohrer, 2009, p. 9), having repeatedly demonstrated applicability across a broad spectrum of domains; for example, including motor skills, verbal learning, and assorted academic content and ranging from early-childhood training through college-level education.

Rohrer (2009) points out that relatively few studies have experimentally examined the spacing effect in the context of spacing mathematics practice. Rohrer and Taylor (2006) reported that they were unable to find a single experiment devoid of confounding flaws in design that studied how the distribution of practice affects the retention of conceptually higher-order mathematics tasks. Dempster (1988) laments the lack of 
widespread application of the spacing effect to school-like tasks and classroom learning. Among the nine obstacles to application Dempster rebuts, he states that perhaps the most serious is the dirth of "programmatic research... in educational settings... involving curriculum design and classroom teaching" (p. 632). Cepeda et al. (2008) posit another reason for the lack of institutional application of the spacing effect. "The fault appears to lie at least partly in the research literature itself: On the basis of short-term studies, one cannot answer with confidence even basic questions about the timing of learning" ( $p$. 1095). Their point and those of Dempster (1988), Rohrer and Taylor (2006), and Rohrer (2009) support the need for a longitudinal-type study of the spacing effect in the context of a community-college mathematics classroom.

Although “... the spacing effect is one of the most studied phenomena in the 100year history of learning research," Dempster (1988) makes a compelling and impassioned argument in defense of further research:

...the spacing effect is neither intuitively obvious, nor well known among educators. Accordingly, it is reasonable to assume that those who become teachers, administrators, curriculum developers, or writers of reading series are ignorant of the spacing effect, just as many psychologists are not clear about the totality of educational situations that call for its application. Thus, our ignorance of actual classroom practice should not be interpreted to mean that widespread implementation of the spacing effect has little or no potential for improving classroom learning...[for] it would be a mistake to do what these comments imply which is simply to stop investigating the phenomenon. Although it may take some clever research to avoid diminishing returns, continued experimental study of the 
spacing effect can yield valuable information regarding its parameters and cause(s). Then too, applied research and widespread application should produce the data base needed to evaluate the particular conditions under which the spacing effect works best. (p. 633)

\section{Deficient-Processing Mechanism and Encoding-Variability Theory}

In tandem with the spacing effect, there are several other theories that not only have bearing on a study of seat-time apportionment in undergraduate courses; they also have been used by some theorists in attempts to explain the cognitive basis for the spacing-effect phenomenon. Two of the most prominent explanations are the deficientprocessing mechanism and encoding-variability theory (Toppino \& Bloom, 2002). They intersect in several respects. The theory of deficient-processing hypothesizes that when one is afforded opportunities for reinforcement of the learning experience that are suitably spaced apart so as to enable adequate processing, the quantity of the information processed and the quality of the memory encoded will be enhanced. Encoding-variability theory is predicated on the notion that multiple, less comprehensive learning sessions - in contrast to fewer, massed sessions - aid retention by giving the learner opportunities to mentally encode the subject matter in a variety of ways. Thus, due to the formation of a greater number of associations, learners generally have a greater likelihood of and duration of recall.

Baddeley (1976) attributes the genesis of encoding-variability theory to several papers in the early 1970s authored by Edwin Martin. Ironically, Baddeley contends that, at the time, Martin was more focused on what he saw as the negative effects of encoding variability, arguing that fewer encodings would increase the probability of recall. 
According to Baddeley, Martin's conception was gradually overturned, though, as others (e.g., Bower, 1972; Glenberg, 1979; Melton, 1970) found merit in encoding-variability theory as an explanation for the spacing effect.

\section{The New Theory of Disuse}

In recent years, other theories have been conjectured, such as Bjork and Bjork's (1992) new theory of disuse. The word new is included to avoid confusion with E.L. Thorndike's old (circa-1914) theory of disuse, which was long ago discredited (Bjork \& Bjork, 1992). Although the scope of Bjork and Bjork's disuse theory and its many premises are far too voluminous to delve amply into here, there are several components of it that are quite worthy of consideration in preparing to design a study of community college seat-time apportionment.

First, the new theory of disuse distinguishes repeatedly between the storage strength and the retrieval strength of items in memory. They contend that storage strength is latent, having no direct relationship to performance. That is, there can be items in memory with high storage strength but low retrieval strength, due to a lack of practical relevance at the time; for example, one's home phone number from a previous residence. These researchers also caution the avoidance of confusing frequent memory retrieval failures, which is a natural part of human memory, with a deficiency in learning. As for how their new theory of disuse helps to explain the spacing effect, Bjork and Bjork (1992) write:

In general, spacing of repetitions results in higher storage strength than does massing of repetitions, which in turn slows the rate of loss of retrieval strength and, therefore, enhances long-term performance. Massing, however, can produce 
a higher initial level of retrieval strength, which, given a short enough retention interval, can result in a higher level of recall than that produced by spaced repetition. (pp. 46-47)

A second aspect of Bjork and Bjork's theory that merits consideration is how the spacing effect may manifest itself differently between learning something new and unfamiliar versus the process of relearning something encountered in the past. The latter is often the case with community-college algebra students. Some of Robert A. Bjork's subsequent scholarship in collaboration with others (e.g., Fritz, Morris, Bjork, Gelman, \& Wickens, 2000) found that when something previously learned has high storage strength, massed relearning can result in higher performance for a certain period of time than spaced relearning (Schneider, 1997).

The implications of this for a study of seat-time apportionment include the need for more reflection upon: (a) ways to avoid commingling measurement of performance in a learning versus a relearning situation; (b) whether a predictor variable attempting to quantify original learning versus relearning for the individual student, based upon, for example, prerequisite coursework and recency thereof; and (c) differences in how the spacing effect may behave in a cross-sectional research design versus a longitudinal one.

\section{Learning-Style and Developmental-Theory Considerations}

In addition to the spacing effect and the explanatory cognitive theories it has spawned, information-processing preferences and social-developmental theories are two areas of scholarly interest that are often difficult to quantify but may weigh heavily upon the variability in effects—-from one student to the next—of seat-time apportionment in undergraduate mathematics courses. Increasingly, researchers have come to appreciate 
the array of symbiotic connections between learning-style theories and developmental theories, for the former usually incorporates aspects of the latter (Evans, Forney, Guido, Patton, \& Renn, 2010).

One particularly instructive example of this interdependency between psychosocial attributes and cognition processes is David Kolb's (1984) theory of experiential learning, which is sometimes referred to in the literature as Kolb's experiential learning model (ELM). Grounded in the experiential works of Piaget, Dewey, Jung, and Lewin, Kolb's ELM consists of two continua. The first is the individual learner's perceptual modes (i.e., how one receives information), which range from the polar extremes of what Kolb terms concrete experience (the "feeling" learner) to abstract conceptualization ("thinking"). The second is the learner's internalization mode (i.e., how one processes information), for which the gamut extends from active experimentation ("doing") to reflective observation (more passively "watching"). Kolb (1984; Kolb, Boyatzis, \& Mainemelis, Experiential learning theory: Previous research and new directions, 2001) views these two spectra as the stages of a circular four-part learning cycle; specifically, learning often begins with a concrete experience (step 1). The concrete experience creates the opportunity for the learner to observe and reflect (step 2), which, in turn, leads to assimilating and distilling the learning into an abstract conceptualization (step 3). The formulation of these conceptualized abstractions results in new implications that lend themselves to active testing and experimentation (step 4).

Finally, as the experiential process of testing new implications creates the opportunity for additional concrete experiences, Kolb's ELM progression spirals back to step 1, and the four-step cycle begins anew. 
Kolb's ELM asserts that, for each of us, our heredity, our prior life experiences, and the needs of our present environment influence our individual learning-style habits. In other words, "We resolve the conflict between concrete or abstract and between active or reflective in some patterned, characteristic ways" (Kolb, Boyatzis, \& Mainemelis, 2001, p. 194). It is helpful to conceive of these two continua of Kolb's ELM (concrete to abstract and active to reflective) as intersecting axes, because doing so lends itself to the formation of four distinct quadrants. In terms of the vernacular of Kolb's Learning Style Inventory (LSI), these quadrants comprise his ELM's four basic learning styles: convergers, accommodators, divergers, and assimilators. In essence, these are four habitual predilections students display when responding to a learning environment. For example, mathematics majors tend to be assimilators (Kolb, Boyatzis, \& Mainemelis, 2001); namely, individuals whose dominant predilections are abstract conceptualization (AC) and reflective observation (RO). Diametrically opposite to the assimilators are the accommodators, who tend toward concrete experience (CE) and active experimentation (AE). Whereas assimilators are more interested in abstract ideas, logical cogency, and contemplation than they are in social interaction and collaboration, the accommodators tend to be action-oriented, application-driven, and trusting of their "gut instinct." More of their learning is reliant upon human interaction.

For purposes of this doctoral research study, Kolb's four-stage ELM and his LSI instrument's associated four learning-style preferences prompts several questions: Do different seat-time apportionment configurations significantly enhance or impede the learning of college-level mathematics in terms of students' progression through Kolb's four-stage spiral learning cycle? Is there a difference among Kolb's four learner-style 
types and their respective degrees of suitability for more frequent but less comprehensive (shorter-duration) class-meeting sessions versus less frequent but more massed (longerduration) class meetings?

Part of the answers to these questions may be found by first considering the predominant pedagogical practices of college faculty and, in particular, mathematics instructors. Although the "sage on the stage" pure lecture mode of instruction is less prevalent than it has been historically, to this day it still remains the predominant coursecontent delivery method for the majority of mathematics, engineering, and science professors (Di Muro \& Terry, 2007; Felder \& Brent, 2005; Jones, Reichard, \& Mokhtari, 2003). In the classroom setting, students who prefer the assimilating learning style are well suited for lecture-based instruction, yet most students are not assimilators and, consequently, favor, other delivery modes.

For example, convergers prefer hands-on, experimentation-type learning experiences, while accommodators and divergers benefit from, among other things, group-collaboration activities. Consequently, since college-level mathematics educators are themselves typically assimilators and they tend to favor lecture-style teaching, it is not surprising that some studies (e.g., Jones et al., 2003) have found that assimilators have a significantly higher grade point average (GPA) than students who are inclined toward any of the Kolb ELM typology's other three learning styles.

Di Muro and Terry (2007) contend that since today's millenial-generation students exhibit a greater diversity of learning-style preferences and intellectualdevelopment experiences than past generations of college students, mathematics educators who refuse to budge out of their exclusively-lecture "comfort zone" may suit 
Kolb's assimilators yet often do a disservice to students with other types of learning-style predispositions.

Therefore, it is reasonable to conjecture whether a community-college mathematics course that meets once per week for a 3-4 hour traditional lecture is even more problematic for the majority of today's community college students, who typically are not assimilators and do not have a track record of academic achievement. Also, the generational differences in learning-style preferences may play a role in the extent, if any, to which longer-duration class meetings at community colleges may be more problematic for younger, first-time-in-college students (who more often enroll in daytime courses) versus older, returning students (who are more often evening-class attendees).

In contrast to the variable of age, which is commonly included in multivariate studies of student persistence, there is another demographic variable that is often omitted from consideration in the literature (Nelson Laird \& Cruce, 2009) yet may be uniquely pertinent to an examination of the relationship between seat-time apportionment and student academic performance at the community college level: the differences between part-time and full-time students. The findings of Nelson Laird and Cruce (2009) are particularly noteworthy in this regard. Based upon their hierarchical linear modeling analysis of a random sample of nearly 56,000 college seniors from 224 participating institutions, they found:

Institutions that enroll greater percentages of part-time students are less engagement-oriented and that the institutional culture at those institutions negatively affects full-time students. However, [their study's results] also suggest that increasing the amount of student-faculty interaction among part-timers will 
benefit those students and may eliminate the small existing difference between full-time and part-time students' self-reported general education gains. Increasing part-time student interactions with faculty will provide benefits to full-timers as well. (p. 311)

As to whether increased student engagement may have longitudinal effects on academic performance, Kuh, Cruce, Shoup, Kinzie, and Gonyea (2008) concluded that:

Engagement has a compensatory effect on first-year grades and persistence to the second year of college at the same institution. That is, while exposure to effective educational practices generally benefits all students, the effects are even greater for lower ability students and students of color compared with White students... suggesting that institutions should seek ways to channel student energy toward educationally effective activities, especially for those who start college with two or more 'risk' factors being academically underprepared or first in their families to go to college or from low income backgrounds. (p. 555)

Such compelling research may prompt one to ask whether scholars have examined the relationship between seat-time apportionment and student engagement and persistence, and, if so, what have they concluded.

\section{Seat-Time Apportionment Studies}

This dissertation author's extensive review of the literature uncovered few studies that have centered their scrutiny upon seat-time apportionment at the postsecondary level. In fact, only two studies that analyzed the issue in a community college or undergraduate university-level mathematics context were located. 
One is the work of Lazari (2007), which was a univariate, retrospective study of differences in retention rates and mean final-exam scores for the population of all Valdosta State University college-algebra students who were enrolled in 3-day-per-week, Monday-Wednesday-Friday (MWF) sections versus 2-day-per-week, Tuesday-Thursday (TR) sections. The MWF and TR classes had seat times of 50 and 75 minutes per class meeting, respectively. The data were collected for each of eight semesters, over a 4-year span. However, only two variables were considered. First, within each semester, all individual final-exam scores were obtained and were disaggregated into two groups: 50minute versus 75 -minute seat times. On a semester-by-semester basis, the mean and standard deviation of the final-exam scores were computed for both groups. The second dependent variable was the proportion of enrollees who completed the final exam, which Lazari viewed as a measure of the rate of student retention. Using extremely basic dataanalysis methods, the researcher then proceeded to perform a two-sample $t$-test upon the mean final-exam scores and a two-sample $z$-test for proportions upon the student retention rates.

Although his hypothesis testing results varied from semester to semester, Lazari's overall conclusions were: (a) The mean final-exam scores for students enrolled in 50minute (MWF) sections was usually significantly higher than for those in 75-minute (TR) sections, and (b) there was in most semesters no statistically significant difference at the .05 significance level in student retention rates between these two seat-time configurations. The author recommended that college algebra be taught only in a MWF format but acknowledged that doing so would likely be logistically impractical. $\mathrm{He}$ 
encouraged a future study comparing student performance and retention in morning classes versus evening ones.

Lazari's study has several strengths, but far more weaknesses. His effort to compile census data over a 4-year span is commendable, for it increased the statistical power of his hypothesis tests. However, the 25-minute-per-meeting seat-time differential between the two groups considered is arguably negligible, which may partly explain why his retention-rate findings did not support the spacing-effect phenomenon. Among the many deficiencies in his study, four are particularly worthy of mention. First, Lazari failed to disaggregate the data on any level other than the semester of enrollment, and he omitted any controls with regard to, for example, the individual students and their backgrounds or, for that matter, the instructors and their pedagogy. These omissions in design and data collection leave a countless number of potentially lurking-variable explanations open to speculation. Second, Lazari failed to provide any rationale for his data-analysis plan, and, moreover, he failed to demonstrate, nor even mention, whether his data satisfied appropriate statistical-distribution assumptions prior to his selection of the univariate techniques he employed. Third, Lazari's research design does not appear to be an outgrowth of theoretical frameworks nor, seemingly, was it reliant upon a contemplative literature review. Fourth, and most notably, it is important to clarify that although Lazari compiled data semester-by-semester for four years, his research design is cross-sectional, not longitudinal, because it only considers one wave of data (i.e., a single test score on a single day). More will be stated about this design limitation shortly.

The other peer-reviewed study of seat-time apportionment of undergraduate mathematics courses utilizes a sample of Florida community college students and has 
been published in two forms: the doctoral dissertation of Odu (2008) and a follow-up journal article authored by Odu and his major advisor Gallo (2009). Like Lazari, Odu conducted an analysis of the spacing effect in a college algebra course setting. In contrast to Lazari's study, the first three (of the four previously mentioned) deficiencies in Lazari's study do not exist in Odu's meticulous research. Odu considered three different weekly seat-time apportionment formats: three 50-minute sessions, two 75-minute sessions, and one 165-minute session with one 15-minute break. Furthermore, in his multilevel multivariate analysis which was accompanied by several analyses of potential interaction effects, he relied upon final-exam scores as his dependent continuous variable, while using an extensive assortment of predictor and control variables. These included an inventory of students' attitudes toward mathematics, an inventory of their learning styles, a pretest of their algebra background knowledge, four unit-exam scores, and six instructor-related attributes. Overall, the theoretical and methodological rationale for each research-design decision was persuasive and presented in a balanced way, accompanied by a refreshingly introspective discussion of the study's limitations and delimitations. With the aid of hierarchical linear regression, the primary conclusions were that (a) college algebra should not be offered in a one-day-per-week configuration, (b) students with pretest scores below 70\% should be enrolled in three-day-per-week sections, and (c) students with certain learning-style preferences are especially ill-suited for the one-dayper-week delivery format.

There is much about Odu's dissertation that is worthy of discussion. However, there are two aspects that are of paramount significance to the purposes of this dissertation research study and its author, as he continues to study the scholarship of a 
growing, distinguished cadre of methodologists (e.g., Raudenbush \& Bryk, 2002; Collins \& Sayer, 2001; Hox, 2010; Little, Schnabel, \& Baumert, 2000; Singer \& Willett, 2003). First, these research-design experts are critical of what they assert is the chronic overuse and misuse of cross-sectional design and analytical methods, which are so pervasive in educational research. In this regard, the first shortcoming of Odu's study that merits special attention is his sample methodology, sample size, and his actions regarding subjects for whom there was missing data. He chose a convenience sample comprised of six college-algebra classes on one campus of a large community college. This decision, presumably, was based upon the difficulties inherent in acquiring consent to administer several inventories to students and a number of confining course-design controls upon instructors. Nonetheless, even at the seat-time apportionment level of disaggregation, having only six class sections may have been a serious design flaw, which could have been overcome. For instance, consider this: After removal of 52 subjects (31\%) from the original sample of 168 students due to either missing final-exam scores or missing data on the learning-styles inventory, the remaining sample of 116 students was comprised of more than twice as many students enrolled in classes with seat times of 75 minutes as there were students with 50-minute and 165-minute seat times combined. Although Gallo and Odu's (2009) contention that the remaining statistical power still exceeded .8 even after losing more than $30 \%$ of their convenience sample of students in six classes, one cannot help but question the generalizability of the study's conclusions to communitycollege college algebra students and whether there was over-fitting in the regressionmodeling process. Perhaps one way that Odu could have countered readers' likely 
concern about both matters would have been to perform a cross-validation procedure (Tabachnick \& Fidell, 2007).

The second and most important point to be made about Odu and Gallo's research, ironically enough, is stated eloquently and forthrightly by Odu (2008) himself:

Although this study found a significant difference between spaced and massed practices in college algebra retention over 1 semester (16 weeks), what are the long term effects of the gain in student achievement? Will this prior knowledge be helpful when students move onto courses that require college algebra as a prerequisite? To answer these questions, it is recommended that a longitudinal study be conducted to measure long-term effects of scheduling on achievement. (p. 189)

This acknowledgement further bolsters the argument put forward in this dissertation that - in a prerequisite-knowledge-driven, sequential-course-based discipline like mathematics - a multilevel longitudinal analysis beginning at the remedial algebra level is required in order to gain valuable insights into the relationship between seat-time apportionment and student learning gains, course success, enrollment persistence, and time to program completion. Furthermore, with regard to Odu's removal of incomplete subjects due to missing data, longitudinal approaches like survival analysis (which also is known as event history analysis, failure time analysis, and hazard modeling) afford researchers an approach called censoring that remedies some of the often disregarded flaws inherent in traditional data-elimination and imputation strategies (Heck, Thomas, \& Tabata, 2010, 2012; Hox, 2010; Singer \& Willett, 2003). 


\section{Summary}

This literature-review chapter addressed five eclectic areas of scholarly activity that bear upon the topic of seat-time apportionment in postsecondary course scheduling. Respectively, the five aspects discussed in this chapter were: (a) the predominant trends and influences related to postsecondary-level course scheduling; (b) how these trends and influences fit within the broader landscape of community colleges' decision-making practices and, in particular, their institutional-research infrastructure and methods; (c) several cognitive learning theories, including the spacing effect, the deficient-processing mechanism and encoding-variability theory, and the new theory of disuse; (d) the role of learning-style and developmental-theory considerations; and, lastly, (e) a review and critique of past seat-time apportionment studies that pertained to community-college mathematics courses.

In brief, this literature review's core contention is: The dearth in existing studies that have examined the relationship between seat-time apportionment in postsecondary mathematics courses and student academic performance supports the need for a study of this dissertation author's stated research problem. In contrast to the two aforementioned cross-sectional studies of seat-time apportionment in college-level mathematics courses, the author of this dissertation remains convinced that a longitudinal research design would be preferable, for it will: (a) enhance the construction of models that are better equipped to account for and explain variations between individual students, between various courses, and between different seat-time apportionment configurations; (b) more appropriately address the effects of missing data and unobserved survival events; and (c) bridge the chasm, to some degree, between qualitative and quantitative research 
methodologies (Ruspini, 2002, p. 26). As Ruspini argues, "The tendency to view the two research traditions as reflecting different epistemological positions and divergent paradigms has exaggerated the differences between them" (p. 26). Fortunately, recent advances in statistical software have made a retrospective-longitudinal research design accessible and practicable for education researchers as well as advanced graduate students (Heck et al., 2010).

Whereas Chapter 2 touched upon the guiding principles behind multilevel longitudinal analysis methods and outlined its main advantages over cross-sectional research designs, the next chapter expounds upon the details of this research dissertation's methodology. 


\section{CHAPTER III \\ METHODOLOGY}

This chapter begins with a reiteration of the study's purpose. It then provides the study's research questions and hypotheses, its research design, information about its setting and participants/subjects, the data-collection procedure, data-analysis techniques, and methodological limitations.

\section{Purpose of the Study}

The purpose of this retrospective-longitudinal study was to investigate whether scheduling courses in large time blocks (e.g., three or more hours once per week) is significantly related to the academic performance of community college students who enrolled in one or more remedial-level mathematics courses offered in this format, as compared to the academic performance of students who enrolled in courses that met more frequently and in medium-length time blocks (of 75 minutes twice per week) or shorterlength time blocks (of 50 minutes thrice per week).

\section{Research Questions and Hypotheses}

The central research question to which this study endeavored to respond was simply: Is there a relationship between seat-time apportionment in community-college algebra courses and course success, course enrollment persistence, and time to complete general-education level mathematics coursework?

The exploration of this comprehensive research question was subdivided into three component questions, in which student success was operationalized based upon the student's final letter grade earned in a given course: 
1. In community-college developmental-level algebra courses, is there a difference in student success for students enrolled in classes that meet one day per week in longer-duration class meetings versus for those enrolled in medium-duration (twice weekly) class meetings versus for those enrolled in shorter-duration (thrice weekly) class meetings?

2. In community-college developmental-level algebra courses, is discrete survival time, as measured by date of withdrawal from the course, related to the number of class meetings per week and the duration of each meeting?

3. Across the sequence of community-college algebra courses, is there a longitudinal difference in the time to complete at least one general-education college-level mathematics course between students who satisfied the prerequisite algebra course requirements by completing one or more algebra courses scheduled in a one-day-per-week, longer-duration format and students whose prerequisite algebra course requirements were entirely fulfilled in courses that had medium-duration (twice weekly) or shorter-duration (thrice weekly) class meetings?

Based upon this dissertation author's literature-review findings, the three research hypotheses for this study were:

$\mathrm{H}_{1}$ : There is a difference in student success for students enrolled in classes that meet one day per week in longer-duration class meetings versus for those enrolled in medium-duration (twice weekly) class meetings versus for those enrolled in shorterduration (thrice weekly) class meetings. 
$\mathrm{H}_{2}$ : Discrete survival time in community-college developmental-level algebra courses, as measured by date of withdrawal from the course, is related to the number of class meetings per week and the length of each meeting.

$\mathrm{H}_{3}$ : Across the sequence of community-college algebra courses, there is a longitudinal difference in the time to complete at least one general-education collegelevel mathematics courses between students who satisfied the prerequisite algebra course requirements by completing one or more algebra courses scheduled in a one-day-perweek, longer-duration format and students whose prerequisite algebra course requirements were entirely fulfilled in courses that had medium-duration (twice weekly) or shorter-duration (thrice weekly) class meetings.

\section{Research Design}

The design of this multilevel, longitudinal study was ex post facto because the independent variable of primary interest (seat time) could not be manipulated by the researcher. In the parlance of various methodologists (e.g., Kerlinger \& Lee, 2000; Newman and Benz, 1998), seat time was therefore considered an attribute variable; in contrast to an active variable, which is an independent variable under the control of the researcher.

Furthermore, seat time was not a form of treatment that could be randomly assigned, since the registration process is such that community college students selfselect the course section(s) in which they enroll—with some choosing their courses autonomously, while others seek assistance, for example, from faculty counselors, academic advisors, academic administrators, and/or family members. Methodologists commonly term this way of assigning subjects to levels of the treatment as endogenous, 
as distinguished from exogenous assignment (Murnane \& Willett, 2011; Shadish, Cook, \& Campbell, 2002). Exogenous assignment refers to subjects' placement into treatment levels being determined by an agent/researcher who is external to the system being investigated, as is the case in experimental —and, it should be noted, also in quasiexperimental - research designs. In contrast, an endogenous assignment is one in which the levels of treatment are due to forces/parties that are internal to the system, which is clearly the case with community college students' assignment to the "treatment" of seattime apportionment levels. Murnane and Willett (2011) eloquently elaborate:

Well-trained researchers recognized that... students placed endogenously in classes of different sizes may differ from each in respects that are difficult to observe and measure.... One common response was to include increasingly larger and richer sets of covariates describing the students... in the statistical models that were used to estimate the effect of treatment on outcome.... Seminal studies published in the 1980s threw cold water on this "control for everything" strategy by demonstrating that regression analyses that contained a very rich set of covariates did not reproduce consistently the results of experiments in which individuals were assigned randomly to different experimental conditions. (pp. 3233)

Morgan and Winship (2007) dubbed this control-for-everything era as "the age of regression," during which, they state, “... the rise of regression led to a focus on equations for outcomes rather than careful thinking about how the data in hand differ from what would have generated by the ideal experiments one might wish to have conducted" (p. 13). 
While in past eras many scholars have characterized randomized experimental research design as "the gold standard," a growing assemblage of social-science methodologists have in recent years pointed out the flaws in that portrayal. For example, Berk (2005) stated, "Randomized experiments rest on more complicated, subtle, and fragile foundations than some researchers appreciate... Textbook requirements are rarely met. Thus, randomized experiments are not the gold standard. But if the truth be told, there is no gold standard" (p. 19).

While some education scholars have marginalized the value of ex post facto design due to its well-documented causal-inference limitation, some ardent and esteemed proponents of experimental-design studies acknowledge that "forms of research other than experiments... can also be of great value. Correlational and descriptive research are essential in theory building and in suggesting variables worthy of inclusion in experiments" (Slavin, 2010, p. 111). It is with that mindset that this dissertation study was approached.

In contrast to the ex post facto design of the large majority of studies in the field of educational research, one additional distingishing factor about this study's ex post facto design warrants reiteration: it was longitudinal, instead of cross-sectional. The many dividends afforded by longitudinal design far outweigh its associated related dataanalysis labor costs. For instance, longitudinal design provides a better way to analyze the relationship, if any, between seat-time apportionment and students' progression through (and success in) compulsory, sequential algebra courses. It enables construction of multilevel models which are better equipped to account for and explain variations between individual students, between various courses, and between different seat-time 
apportionment configurations; while, at the same time, providing means by which to more appropriately address the effects of missing data and unobserved survival event occurrences (e.g., persisting in a course or program versus withdrawing, or successfully completing a particular course or program within a finite longitudinal tracking period versus not). Simply stated, learning by its very nature is a growth experience; growth experiences require change over a period of time; longitudinal-type analysis methods equip researchers with a more powerful toolkit by which to quantify differences in growth over time than do cross-sectional methods.

\section{Institutional Context and Its Significance}

Historically, the overwhelming majority of scholarly research on college student attrition/retention has relied upon single-institution data, which, by its very nature, is unable to assess the relative influence of institutional context on student persistence. Titus (2004) contends that even many multi-institution persistence studies have failed to properly examine the role of institutional context. In large measure, he attributes this research deficiency to two main types of statistical-technique misuse. First, many past persistence studies employed single-level statistical methods, which all but ignored the impact of student-level variables operating/nesting within institutional-context (higherlevel) variables. Second, even in the case of those studies that did analyze the impact of institutional context in a multi-level way, most ignored the effect of student variables on institutional persistence rates. Consequently, Titus cautions, “... policy makers may be using institutional persistence rates to make inappropriate judgments about institutional effectiveness and performance" (p. 674). 
Although this dissertation study was reliant upon a data set from a single institution and, hence, was unable to include institutional-context covariates, it is nonetheless important to include mention of at least some of these lurking factors. While a more extensive review of demographic-type variables is provided in Chapter 4, it is important to bear in mind two institutional features which may have affected this study's findings.

First, the implications of the term "open-door institution" are particularly important. While it is commonplace for 2-year public colleges, including the one examined in this study, to have no entrance/admissions requirements (other than a highschool diploma or equivalent), the institution used for this study exhibited a somewhat anomalous characteristic with regard to registration requirements for students whose scores on placement tests warranted enrollment in developmental-level courses. Specifically, in contrast to the student-registration practices at other Florida community colleges during this study's time period, students at this particular South Florida college who placed into developmental-level coursework during at least the first half of this study's 6-semester longitudinal tracking period were largely unhindered from selfenrolling in college-level coursework in other disciplines. This is noteworthy because it may have altered the results of the third research question's analyses. This matter is elaborated upon in Chapter 5.

Second, the institution's locality contains an atypically high amount of postsecondary institutions within a tri-county area, including three large multi-campus 2year colleges, two large state universities, in excess of six private universities, a large assortment of proprietary-type postsecondary institutions, and various public vocational- 
technical institutions. Consequently, it is not uncommon for this college's students to enroll in multiple institutions, even simultaneously within the same semester. The large number of postsecondary educational options available to students, along with the heightened level of inter-institutional competition for market share, only compounded the difficulty in accurately tracking students across a longitudinal timespan.

\section{Population and Sample}

As implied by the wording of the research questions themselves, the population of interest to this study was defined as community college students who enrolled in one or more developmental-level mathematics courses (e.g., a pre-algebra and/or a beginningalgebra course). For many years, the Florida Department of Education's Statewide Course Numbering System (SCNS) identified two sequential courses as developmentallevel mathematics, albeit with some variation in course title: MAT0012 Pre-Algebra and MAT0024 Elementary Algebra, in addition to a combined version of these two courses. In the fall of 2011, these two courses were renumbered and given statewide uniform titles: MAT0018 Developmental Mathematics I and MAT0028 Developmental Mathematics II, respectively. Since students are not awarded college-level credit for successful completion of such courses, nearly all enrollees in developmental-level courses were required to complete them, due to their low placement-test scores. However, on relatively rare occasions, there are students who, for refresher purposes, voluntarily opt to enroll in a developmental-level course, despite their having high enough placement scores to enable them to bypass such coursework.

It should be noted that the researcher decided not to impose any other additional restrictions or qualifications for student inclusion within this defined population. As such, 
this resulting eclectic superset encompasses a wide variety of community college subsets, including (yet far from limited to): various degree/certificate-seeking statuses, as well as non-degree (transient) students; first-time-in-college (FTIC) traditional-age college students as well as older, returning adults; students who placed into developmental-level reading and/or English and/or EAP/ESL courses for non-native language speakers, as well as those who were exempt; and so on.

The specific sample selected to study this population was an entire multi-campus, college-wide census of a cohort of students who enrolled in a Fall-2001 section of MAT0024 Elementary Algebra at a large, urban community college located in South Florida. In that Fall-2001 semester, this institution scheduled a total of 120 class sections of MAT0024, spread among a total of six locations; namely, three large campuses, two satellite centers, and one off-campus facility. All enrollees $(N=3,284)$ within these 120 classes were longitudinally tracked over a retrospective period of two full academic years. An academic year is made up of three semesters: fall, winter, and summer. Thus, the study's duration of two full academic years encompassed six academic terms, beginning with the start of the Fall-2001 semester and culminating with the end of the Summer-2003 semester.

Every member of the sample was tracked over the retrospective period in each of their subsequent mathematics courses (including any repeated attempts in a given course), as well as being tracked in their general enrollment progress toward fulfillment of their declared degree/certificate program requirements (when applicable). Moreover, it should be noted that some of the data collected either pre-dated or post-dated the 2-year 
longitudinal tracking period, in order to shed light on students' pre-2001 individual academic histories and their post-2003 degree-attainment outcomes.

Lillibridge (2008) is one who heralds the value of longitudinal tracking of students, while cautioning that "Anyone conducting or evaluating longitudinal studies should be aware that no student information system is perfect... researchers must weigh the costs of pursuing and achieving data perfection against the benefits" (p. 20). While the validity and reliability of any data set is always a concern, the types of institutionallyarchived, student-records data acquired for this study are known to have a higher degree of reliability relative to many other collection methods and data sources. Furthermore, in some respects, the retrospective nature of the study further boosted the data's reliability; for example, the institution's deadlines for students enrolled in 2001-2003 courses to submit grade-appeal applications or tuition-refund petitions would have long expired. Therefore, any student-record corrections would have been made prior to the collection of the six (semester-length) waves of data.

There was a dual rationale for selecting a 2-year (6-semester) longitudinal period, instead of a longer or, for that matter, short duration. First, a 2-year period is the amount of time successful full-time students are expected to need to complete their communitycollege degree program. Second, six waves of data is consistent with commonly accepted guidelines, when attempting to model change with a discrete-time occurrence data set (MacCallum \& Kim, 2000, p. 52; Singer \& Willett, 2003, pp. 9-10). Fewer waves tend to restrict the types of models that can be tested and may confound measurement error with actual change. Too many waves, on the other hand, can become unwieldy, especially in terms of data collection. Also, "the ability of a model of linear change to explain data will 
likely deteriorate as the number of time points increases well beyond five or six" (MacCallum \& Kim, 2000, p. 52).

As for why the MAT0024 Elementary Algebra course was selected instead of the other developmental-level course (i.e., MAT0012 Pre-Algebra), there were three main reasons. First, MAT0024 is viewed as a high-volume, high-demand course, whereas MAT0012 is not. Said differently, every semester, there are several times as many MAT0024 classes offered as MAT0012's, and for multilevel studies like this one, statistical power is increased by having as many units at the highest level of the data hierarchy (Snijders, 2005). Second, the curricular content of MAT0024 is entirely grounded in algebraic concepts and skills, whereas a large portion of MAT0012 is nothing more than a rudimentary arithmetic-skills review. Thirdly, and most importantly, in order to receive a passing grade in MAT0024, a student had to pass a mandatory state exit exam, in addition to satisfying the instructor's grading criteria. This statewide requirement went into effect in 1998, and lasted until 2011. Among postsecondary mathematics courses in Florida, MAT0024 was unique in this regard, which, therefore, made its selection for this study an easy decision. That is because having a standardizedtest benchmark of this type markedly increases the internal consistency—and, hence, the reliability — of the dependent variable (DV) course success across classrooms and instructors, as compared to such a DV's internal consistency in any other mathematics course.

There were four primary factors that influenced the decision to select this particular decade-old longitudinal time period instead of a more recent one: 
- It enabled seat-time apportionment to be operationally defined as a trichotomous independent variable, instead of just a binary one, due to the fact that this 2001-2003 timespan contained the greatest variety of (and balance among) long-, medium-, and short-duration seat-time class sections. As a result, this increased the power of the statistical tests used in this study's multilevel analyses. A more recent time period would not have afforded that benefit, because in 2006 the community college selected discontinued the Monday-Wednesday-Friday (MWF) 50-minute (short) seat-time apportionment option. Driven by budgetary-constraint and market-competitiveness considerations, the college's leadership instituted what was described as a more standardized "block scheduling model," one that various Florida postsecondary institutions implemented roughly around the same time. The MWF 50-minute classes were replaced with Monday-Wednesday (MW) 75-minute classes, which mirrored the longstanding Tuesday-Thursday (TR) 75-minute (medium) seat-time configuration. A relatively small number of Friday-only (i.e., one-day-per-week) course offerings were scheduled in select buildings, with the stated objective at the time being that taking some buildings "off line" on Fridays would yield a significant savings in facilityrelated costs.

- From Fall-2003 through Summer-2005, the researcher was in an administrative position at the institution and chose to initiate a major redesign of the master schedule of mathematics course offerings at their largest major campus and at one of their satellite centers. With the exception of weekend course offerings and one offsite course section, his redesign resulted in the virtual elimination — at, most notably, the developmental course level—of this study's most important seat-time level: the one- 
day-per-week, 150-minute-or-longer (long seat-time) class. Consequently, that 20032005 time period would not have been well suited for this study.

- A more recent longitudinal tracking period would have complicated the research design by confounding two distinct class-section (Level-2) variables: a course's seattime duration per meeting with its scheduled calendar length. That is to say, the community college from which the data set was obtained has in recent years gravitated toward a course-scheduling model that has greatly expanded the number of compressed mini-term course offerings, especially with some recent student-success initiatives pertaining to developmental-level courses. Like a number of its community college peers, this institution's decision to rely more heavily upon mini-term course scheduling has, in part, been motivated by market-force considerations, for several large proprietary institutions are well known for this type of scheduling design. The 2001-2003 time period selected enabled the researcher to largely avoid the complication of mixing, for example, 16-week full-term MAT0024 classes with greatly condensed 8-week mini-term offerings.

- A more recent time period potentially would have added a set of unwieldy technology-related confounding variables. That is to say, in more recent years, developmental-level mathematics students have had a much wider menu of technology-enhanced classes to choose from. For example, some classes are fully online. Others are scheduled in a blended/hybrid way. Even in the case of traditional face-to-face lecture-style sections, some campuses mandate that all their faculty and students integrate into the course (including in the computation of course grades) an online course-management system that contains algorithmically-generated homework 
and online-quiz problems, a wide array of supplemental videos, other multimedia learning-tool enhancements, 24/7 electronic tutoring resources, and so forth. In short, the array of course configuration types is nearly unlimited. Even though this study's 2001-2003 tracking period includes some of this variability, it was far less prevalent and varied; hence, it was easier to account for it in the analysis.

\section{Setting and Participants}

As for the setting of this research study and the degree of interaction with the members of its sample, four clarifications are in order. First, there were no treatments for the student subjects nor was there any contact in any manner between the researcher and the subjects. Hence, this study did not in any way entail any subject-recruitment activities. All subject information was provided by the college's Office of Institutional Research, Planning, and Effectiveness (IRPE), in collaboration with the District Registrar's Office. Second, there was no use of student names, addresses, phone numbers, or the like. For longitudinal tracking purposes and to facilitate the communication of any follow-up inquiries the researcher made, the IRPE staff assigned every student member - as well as every instructor — a unique dummy identification code number. Hence, there was no possible risk whatsoever of information about any individual subject being reported in the study and/or released to any third party. Third, no instruments, inventories, or the like were utilized. So, there was no need to perform preliminary research activities such as pilot testing of instruments and estimating of their reliability. Fourth, and finally, the purpose of this study was purely educative. As such, there was no direct risk (nor benefit) to the subjects involved. Therefore, on the basis of these four criteria, Florida International University's Office of Research Integrity (ORI) 
determined that this research did not meet the definition of human subject research (HSR) and, accordingly, did not require submission to nor approval of Florida International University's Institutional Review Board. The ORI's official non-HSR determination letter is shown in Appendix A.

\section{Data Collection Procedure}

Written requests for mainframe database access to student-records data were submitted to the aforementioned community college. After several meetings with the college's academic- and student-affairs senior-level administrators, the institution's Vice President of Academic Affairs issued a formal approval letter to the researcher on January 15, 2011, granting him access to the archival student-records information requested. Subsequently, after additional meetings and the submission of more detailed documentation, it was agreed that the interests of all parties concerned would be best served by assigning the technical/programming aspects of the data collection to the college's Office of Institutional Research (IR). The IR staff then subdivided this electronic data-collection project into what ultimately became 17 individual data files, which were issued to the researcher in five separate phases over a 4-month period, during the summer/fall of 2012. After the accuracy of each data file was cross-checked by no fewer than two IR personnel, it was made accessible to the researcher in commaseparated values (CSV) file format, via one of the institution's password-secured internet sites.

With the aid of SPSS version 20 as well as Microsoft Excel 2010, the researcher then performed the necessary merging, restructuring, filtering, recoding, and recomputing of the variables contained within the $17 \mathrm{CSV}$ files provided by the college's 
IR staff. To ensure that the integrity of the data set was not compromised by so much merging and restructuring of the original CSV data files, a variety of if-then conditional variables were created to cross-check the accuracy of each file-merge operation. Other safeguard measures, such as frequent cross tabulations, were also implemented.

\section{Variables}

The data were collected and analyzed at two hierarchical levels: (a) the individual student level (Level-1), which encompassed each subject's institutional enrollment history, academic history, and assorted other demographic- and academic-related covariates; (b) the class/section level (Level-2), including, for example, the day(s), time, and duration of class meetings, instructor demographics, and aggregated student variables.

In the case of the first research question, its dependent variable (DV) was at Level-1 and pertained to whether or not the student earned a passing grade (of " $\mathrm{C}$ " or higher) in the Fall-2001 MAT0024 Elementary Algebra course. This binary outcome was coded as $1=$ Successful and $0=$ Unsuccessful. This research question's independent variable (IV) of primary interest was an ordinal Level-2 variable: the seat-time level of the MAT0024 class section in which the student was enrolled. This was trichotomously defined as short, medium, and long, which were then coded zero through two, respectively. Based upon the literature review and in accordance with research hypothesis $\mathrm{H}_{1}$, the short seat-time level served as the analysis's reference category. It should be noted, however, that the researcher also experimented with a binary coding of the seattime IV. In fact, this alternative dichotomous approach was explored in two ways: (a) coding seat time as short versus not-short; and (b) coding it as long versus not-long. 
Chapter 4 includes details about some unanticipated intricacies in coding some anomalous course sections' seat-time level, which arose during the data-analysis stage of this research.

The second and third research question's entailed survival-analysis methodology. While both questions shared the same grouping variable (namely, the aforementioned Fall-2001 MAT0024 class's seat-time level), they differed with regard to their respective time-metric and hazard-event definitions. More is discussed about this later in Chapter 3 and, additionally, in Chapter 4.

\section{Level-1 Variables}

With the exception of the dummy identifier code randomly assigned to each student by the institution's IR staff, the student-level (Level-1) variables fell into one of the following four categories (listed in no particular order):

1. Non-academic attributes (at the time of Fall-2001 enrollment) - (a) date of birth (DOB); (b) sex (Male, Female); (c) ethnicity (Hispanic, Non-Hispanic, Unreported); (d) race (Asian, Black/African-American, Native American, Pacific Islander, White, Unreported); (e) country of birth; (f) immigration status; (g) verified disability-services status (disabled versus not disabled); (h) need-based financial-aid status (i.e., based upon whether Pell grant was offered to student); and then (i) student age was computed by subtracting DOB from the MAT0024 class's session-start date.

2. Academic variables pertaining to student's MAT0024 Fall-2001 enrollment-(a) course grade; (b) course withdrawal date, when applicable; (c) tuition/fee payment date; (d) tuition status (in-state/resident versus out-of-state/nonresident rate); (e) fulltime or part-time enrollment status; and then (f) time-to-course-withdrawal (for 
Research Question \#2's survival analysis in which the time metric's unit size was days) was computed by subtracting the MAT0024 class's session start date from the student's course withdrawal date.

3. Academic variables pertaining to student's subsequent mathematics-course enrollment at the institution during remainder of two-year longitudinal tracking period - (a) each attempted course's title; (b) its course prefix-number code; (c) its section reference number; (d) semester and session-within-semester in which course was attempted (as two separate variables); (e) student's course grade for each subsequent mathematics course attempted; and, then, computed variables included (f) total number of mathematics course attempts during tracking period; (g) total number of mathematics-course successes (i..e., course grades of "C" or higher); (h) total number of general-education mathematics courses attempted; (i) total number of general-education mathematics course successes; and (j) time elapsed (measured in semester units) after Fall-2001 semester until student's first success in a generaleducation mathematics course.

4. Other academic-related covariates - (a) six variables pertaining to all previous mathematics course enrollments at the institution prior to the Fall-2001 semester, including course prefix/number, course title, course grade, when it was taken (both the semester and the session within the semester), and the section's assigned courseregistration reference number; (b) six variables pertaining to all previous mathematics course enrollments at other institutions prior to the Fall-2001 semester, including course prefix/number, course title, course grade, when it was taken (both the semester and the session within the semester), and the section's assigned course-registration 
reference number; (c) total number of semester-hour credits attempted and total number earned (i.e., as two separate variables) at the institution prior to Fall-2001 semester, which marked the beginning of the longitudinal tracking period; (d) total number of semester-hour credits attempted and total number earned (also as two separate variables) at other postsecondary institutions prior to Fall-2001 semester; (e) total number of credits attempted and total number earned at the institution and also at other postsecondary institutions (i.e., four separate variables) by the end of the Fall2003 semester, which was the end of the longitudinal tracking period; (f) high-school (HS) diploma/award type; (g) HS diploma/award date, which, in turn was used to compute (h) months elapsed between session-start date of Fall-2001 MAT0024 and HS diploma/award date; (i) whether student attempted a student-life/learning-skills (SLS) course at any institution prior to Fall-2001 semester; (j) whether student passed an SLS course at any institution prior to Fall-2001 semester; (k) test-score based mathematics placement type, reading placement type, and English/writing placement type (three values per each of these three variables: developmental-level, collegeready, or no test score on which to judge); (1) three variables - basis for mathematics placement (e.g., SAT, SAT I, ACT, ECPT, departmental-exam placement, and so on), accompanied by test score and test date; (m) reading-prep placement level (low REA0001c/0007c or high REA0006c/0017c, with blank representing either collegeready status or no test score ); (n) reading-prep satisfaction status (entirely, partly, or not at all) as of start of Fall-2001 semester; (o) English/writing-prep placement level (low ENC0010/0015 or high ENC0021/0025, with blank representing either collegeready status or no test score ); (p) English/writing-prep satisfaction status (entirely, 
partly, or not at all) as of start of Fall-2001 semester; (q) non-native speakers' English-for-Academic-Purposes (EAP) completion status as of start of Fall-2001 semester (entirely, partly, or not at all); (r) three variables pertaining to student's declared program-major at the time and degree-certificate award type sought $(\mathrm{AA}=$ Associate of Arts, $\mathrm{AS}=$ Associate of Science, $\mathrm{ATC}=$ Advanced Technical Certificate, ATD=Applied Technical Diploma Certificate, Noncredit-audit, Nondegree seeking, Nondegree-previously graduated, PSAV=Postsecondary Adult Vocational Certificate, $\mathrm{TC}=$ Technical Certificate, Transient); and, finally,(s) it should be noted that six additional variables pertaining to students' subsequent institutionaltransfer characteristics and degree attainment were collected but were ultimately not incorporated into this particular study - specifically, the institution name, whether it was a 2-year or 4-year school, enrollment start date, enrollment end date, graduation date, and degree/award type.

\section{Level-2 Variables}

As for the class-section (Level-2) variables, they were organized into two main types (once again, listed in no particular order):

1. Attributes of Each Fall-2001 MAT0024 Class Section - (a) class section's assigned course-registration 6-digit reference number (used as a nominal variable in the multilevel analyses for Level-2 hierarchical grouping purposes); (b) class section's session within the term (i.e., 16-week Session I, 12-week Session II, 8-week Session IV); (c) class time block (day, evening, weekend); (d) course-scheduling notes (to ascertain whether section had special/atypical pedagogical approaches and/or anomalous class-meeting schedule; (e) instructional method (e.g., traditional, fully- 
online, and other types defined by researcher after review of each section's coursescheduling notes); (f) class enrollment limit (i.e., maximum class size); (g) class start date (which was, in actuality, the session start date); (h) class (i.e., session) end date; (i) class meeting start time, end time, and day(s) of the week meetings were scheduled; (j) number of class meetings per week; (k) location code (i.e., specific campus/center or offsite location); and, then, the variables subsequently computed were (1) total number of class meetings (calculation based upon course session, specific section's weekly meeting days, Fall-2001 academic/instructional calendar, and institution's Fall-2001 final-exam schedule); (m) seat-time duration (calculated by subtracting start time from end time when all meeting days had uniform start-end times throughout the week, but exceptions were calculated as arithmetic mean of daily meeting times); and (n) number of enrollees (by first creating a frequency distribution file comprised of students per course-section reference number, and then merging in SPSS the resulting file with each student subject's record).

2. Attributes of Instructors of Fall-2001 MAT0024 Class Section(s) - (a) randomlyassigned dummy identification code; (b) sex; (c) ethnicity; (d) race; (e) DOB; (f) tenure status at start of Fall-2001 semester (tenured versus non-tenured); (g) date tenure was awarded; (h) full-time faculty or part-time adjunct-faculty status (which was ascertained from the pay-method code instructor was assigned for the Fall-2001 MAT0024 course, which reflected type of compensation awarded); (i) instructor's institutional hire date(s) accompanied by corresponding full-time/part-time status code (note: there were as many as six listed hire dates/codes for a given instructor, due to their having departed from- 
but later having returned to - the institution on multiple occasions); and then (j) instructor age was computed by subtracting DOB from the MAT0024 class's session-start date.

It should be noted that Level-1 and Level-2 covariates that were continuous (e.g., ages) were recoded as grand-mean centered variables in order to enhance their interpretability, in light of the logit transformation inherent in the first research question's multilevel logistic-regression analysis.

\section{Data Analysis Techniques}

The two primary statistical techniques employed in the analysis of this study's data set were: (a) multilevel (hierarchical) logistic regression for the first research question; and (b) discrete-time survival analysis (DTSA) for the second and third research questions.

\section{General Overview and Rationale}

To analyze the multivariable data pertinent to the first of this study's three research questions (namely, the relationship between seat-time apportionment and academic performance in a developmental-level algebra course), both single-level and multi-level (hierarchical) logistic regression were employed, and the results of these two approaches were compared.

Logistic regression was chosen for several reasons. First, it was an almost axiomatic choice in light of this study's dependent variable (DV) of primary interest (namely, course success) being a binary outcome. Second, in contrast to other techniques that are multivariate (i.e., multiple DVs) and/or multivariable (multiple IVs), such as discriminant analysis, logistic regression is more robust against violations of assumptions pertaining to the distributions of predictor variables. For example, logistic regression 
does not require that IVs be normally distributed, linearly related, nor of equal variance (homogeneity) within each group. Third, logistic regression is a method that permits the independent (predictor) variables to be of various data types and measurement levels, which was certainly the case with this study's research plan (Hox, 2010; Hosmer \& Lemeshow, 2000; Tabachnik \& Fidell, 2007). Fourth, and finally, there is broad flexibility nowadays among the variety of easily-accessible statistical software programs with respect to the model-building menu of options available, in particular, for logisticregression analysis.

To address both the second research question (i.e., the relationship between seattime apportionment and course attrition, as measured by course withdrawal) and the third research question (i.e., whether the seat-time apportionment in a developmental-algebra course on academic performance was longitudinally related to the time until successful completion of a general-education level mathematics course), discrete-time survivalanalysis (DTSA) methodology was employed.

The term survival analysis encompasses a family of statistical techniques and is known by a number of other names; for example, failure analysis, hazard modeling, timeto-event analysis, and event-history analysis. There were three main reasons why it was ideally suited to this study's second and third research questions. First, survival analysis utilizes technique known as censoring, which is a conceptually superior way to address event nonoccurrence (e.g., not all students experience the event of withdrawing from a course or program). Specifically, this research entailed right censoring; namely, with respect to the time to student withdrawal from the Fall-2001 MAT0024 course (in Research Question \#2) and time to complete successfully one's first general-education- 
level mathematics course. Second, survival analysis's main constructs (e.g., life tables and survivor/hazard functions) facilitate better modeling of retention/attrition and, in turn, their relationships to predictors and differences between groups. Thirdly, survival analysis is readily adaptable to multilevel modeling (Heck et al., 2010; Hox, 2010; Singer \& Willett, 2003; Tabachnick \& Fidell, 2007).

\section{Survival-Analysis Constructs and Considerations}

Singer and Willett (2003) recommend a mnemonic phrase to aid researchers in deciding whether a research question warrants the use of survival-analysis methodology. They "refer to [it] as 'the whether and when test.' If your research questions include either word - whether or when-you probably need to use survival methods" (p. 306). This study's second research question is both a whether question (i.e., whether or not students withdraw from the course) and a when question (i.e., when in the semester do course withdrawals occur). Thus, survival analysis was the best-suited methodology for this research question; in particular, discrete-time survival analysis (DTSA), in contrast to continuous-time survival analysis.

As Singer and Willett (2003) point out, this discrete-versus-continuous distinction is vitally important for a multitude of reasons:

Distinguishing between continuous- and discrete-time data is more than a methodological detail. Almost every feature of survival analysis - parameter definition, model construction, estimation, and testing — depends on the metric for time.... Unfortunately, continuous-time methods break down when event times are highly discretized due to a problem known as "ties"... With continuous-time 
data, the probability that two or more individuals share an identical event time (are "tied") is infinitesimally small. (p. 314)

In the case of this dissertation study's second research question, there were 48 ties; that is to say, 48 distinct calendar dates on which two or more students were assigned the withdrawal (W) grade. In fact, nearly $42 \%$ of the 594 student withdrawal grades occurred on three specific dates alone: 10/29/01 $(n=49), 10 / 30 / 01(n=64)$, and 12/18/01 $(n=$ 135). There are two reasons why this high rate of ties was not surprising. First, time was measured in days, and the total number of course withdrawals $(N=594)$ was five-times greater than the 118-day duration of this survival analysis's maximum-possible time period. Second, student-initiated withdrawal from a course is a survival event that inherently includes an institutionally-mandated deadline (e.g., 10/30/01 for all fullsemester Fall-2001 courses), which accounts for the heavy clustering of survival-event times in late October and at the mid-December end of the semester.

Tabachnick and Fidell (2007) divide the family of survival/failure analysis techniques into two goal-type categories. The first category's primary aim is to describe the proportion of cases surviving at various times and, when applicable, to quantify and statistically test for significant survival-time differences between groups. The second type of survival analysis is an extension of the first, in that it examines whether survival times still differ among groups after controlling for other covariates, including any treatment IV of interest. "These are basically regression procedures in which survival time is predicted from a set of variables...” (p. 506). This dissertation study's second research question falls into Tabachnick and Fidell's first survival-analysis category because the question's objective was to determine whether a significant difference in the respective proportions 
of MAT0024 Elementary Algebra students withdrawing at various times during the semester existed among the three seat-time apportionment groups, without regard to other covariates or treatments. Nonetheless, Chapter 5's discussion includes recommendations for future studies that incorporate covariate predictors into the survival analysis.

\section{Preparation of Data Set for Discrete-Time Survival Analysis (DTSA)}

Survival-analysis methodology is predicated on the DV being the time elapsed until some event occurs. Therefore, it demands three constructs be clearly defined from the outset: a target event to be studied; an initial starting time (at which no subject has yet experienced the target event); and a suitable, meaningful metric for clocking time (Singer \& Willett, 2003).

The target event's definition and related considerations. For this study's second research question, the particular target event was a student's withdrawal from the Fall-2001 MAT0024 Elementary Algebra course. The institution records such a course grade with the letter "W" and affixed to that grade is the calendar date the $\mathrm{W}$ was assigned. It is important to bear in mind that, during this study's two-year longitudinal period (of Fall-2001 through Summer-2003), the college policy enabled two types of course withdrawals: student-initiated and instructor-initiated. Unfortunately, the student's academic record and the institution's letter-grade system fail to provide an infallible means by which to distinguish between the two withdrawal types. This limitation is likely to have compromised to a certain extent the reliability of the $\mathrm{W}$ grade—and its affixed withdrawal date — as a measure of course attrition.

For a student-initiated withdrawal, the student either would have had to withdraw online or in-person at any of the institution's registration offices, which are located at 
every one of its campuses and centers. The State of Florida requires each public college to set a deadline for every full semester and for every mini-session within each semester, and that withdrawal deadline must not "... exceed 70 percent of the term. Withdrawals after that date would be granted only through established institutional procedures." (State Board of Education, 1998/2000/2004). The institution from which this study's data were obtained has had a longstanding policy of allowing the student to withdraw without academic penalty prior to the $60 \%$ point in the session. Table 1 summarizes what this meant in practical terms for the 120 class sections of MAT0024 among which the 3,284 Fall-2001 cohort students were enrolled.

Table 1

\begin{tabular}{|c|c|c|c|c|c|}
\hline \multirow[b]{2}{*}{$\begin{array}{c}\text { Session } \\
\text { Type/Code }\end{array}$} & \multirow[b]{2}{*}{$\begin{array}{c}\text { Session } \\
\text { Start Date }\end{array}$} & \multirow[b]{2}{*}{$\begin{array}{l}\text { Session } \\
\text { End Date }\end{array}$} & \multirow{2}{*}{$\begin{array}{c}\text { Student } \\
\text { Withdrawal } \\
\text { Deadline }\end{array}$} & \multicolumn{2}{|c|}{ Session Duration } \\
\hline & & & & Calendar & Full \\
\hline $\begin{array}{l}\text { Full-Semester } \\
\text { Session I }\end{array}$ & $8 / 22 / 01$ & $12 / 18 / 01$ & $10 / 30 / 01$ & 118 & 16 \\
\hline $\begin{array}{l}\text { Mini-Term } \\
\text { Session III }\end{array}$ & 9/19/01 & $12 / 18 / 01^{*}$ & $11 / 9 / 01$ & 90 & 12 \\
\hline $\begin{array}{l}\text { Mini-Term } \\
\text { Session IV }\end{array}$ & $10 / 18 / 01$ & $12 / 18 / 01^{*}$ & $11 / 26 / 01$ & 61 & 8 \\
\hline
\end{tabular}

Among these three session types, the full semester (16-week) type predominated, with $2,700(82.2 \%)$ of the cohort students enrolled in one of the $99(82.5 \%)$ Session I classes. The next most frequently occurring session type was the 12-week (Session III) mini-term, of which there were $20(17.2 \%)$ classes containing a total of 566(17.2\%) students. Lastly, there was one Session IV (8-week mini-term) class, comprised of 18 Fall-2001 cohort students. However, it should be noted that this Session IV class actually had a total of 20 
enrollees, but two were not counted in this research question's analysis because they had withdrawn from their Session I class early enough in the semester to enable them to reattempt the course within the same Fall-2001 semester by enrolling in the 8-week Session IV class. To avoid double counting these two students, only their initial (Session I) attempt was counted in this research question's analysis. The Session IV attempt was classified as a subsequent cohort math class, for purposes of the third research question's longitudinal analysis.

As for an instructor-initiated withdrawal, the institution's policy during this study's time period gave all instructors the right to input a withdrawal grade (W) for a student at any point during the semester, up to and including the College Registrar's endof-term grade-submission deadline. In other words, instructors had the latitude, if ever and whenever desired, to manually withdraw a student after the student-initiated deadline (i.e., at the $60 \%$ point in the course's session). For example, it is conceivable that some instructors may have decided that a given student's violations of the instructor's class attendance policy warranted automatic withdrawal from the course. Other instructors, for instance, may have circumvented the spirit of the college's course-withdrawal policy by improperly granting $\mathrm{W}$ grades to hardworking students who performed well for much of the semester yet ultimately did not attain a passing score on the course's state-mandated exit exam, thus warranting the issuance of the non-passing grade of D or F. The researcher can attest to the fact that historically the vast majority of this particular college's mathematics faculty have been less prone than faculty in other disciplines to award instructor-initiated withdrawals, especially with regard to using the $\mathrm{W}$ as a false surrogate for the F. Many view course grades (A through F) as comprising the totality of 
the instructor's purview, whereas registration statuses (withdrawals, audits, and the like), in contrast, are solely within the student's domain.

Nonetheless, the potential for conflation of student-initiated and instructorinitiated course withdrawals - and the difficulty in quantifying the extent to which the latter may have occurred - remained a concern throughout this portion of the analysis. One way in which the researcher attempted to disaggregate the instructor-initiated withdrawals was to remove any withdrawal events containing dates that were past the student's withdrawal deadline for that particular course/session. However, that remedy was deficient in several respects. For instance, it would have failed to exclude instructorinitiated withdrawals, if any, that were inputted with retroactive withdrawal dates which preceded the student deadline. Furthermore, it would have excluded erroneously any student-initiated withdrawal that were technically performed by the instructor; that is, any instructor-inputted withdrawals after the student deadline that were performed by the instructor solely due to the student requesting to be withdrawn, perhaps based upon extenuating circumstances or perhaps not. More is stated in Chapter 5 about this particular limitation of the study.

The initial starting time - definition and related considerations. For each MAT0024 Fall-2001 student, the start of the survival analysis' semester-long time period was operationally defined as the academic calendar's first day of classes for that particular class's session. In the case of the 99 Session I classes, this meant the clock started on Wednesday, August 22, 2001, as shown in Table 1. Similarly, the starting time for students who were enrolled in any of the 20 Session III classes was defined as Wednesday, September 19, 2001. Likewise, Thursday, October 18, 2001, was the clock 
commencing time for the 18 cohort students in the one Session IV class (i.e., excluding the aforementioned two students who were removed due to having already withdrawn earlier in the semester from a Session I class).

In the case of all three session starting times, it should be noted that the session starting time is not necessarily identical to a given class's first day of class. For example, a Session I class that met only on Saturdays would have had its first scheduled class meeting on Saturday, August 25, 2001, which was three days later than the Session I starting date of Wednesday, August 22. The largest possible difference between a session's starting date and any of its class's first possible meeting date would always be less than 7 days; that is, the largest difference for Session I courses would occur for Tuesday-night-only classes, which would have had their first class meeting on August 28, six days after the August 22 start of Session I.

However, there were two reasons why a potential disparity of up to six days was ultimately deemed trivial enough to be disregarded. First, although the actual starting dates vary from class to class (and, for that matter, ending dates as well), all classes within a given academic session shared a uniform add-drop-refund deadline date (e.g., Tuesday, August 28, 2001 for the Session I classes), as well as a common end-of-term date (e.g., December 18). Consequently, for survival-analysis methodological purposes, it made sense to synchronize the starting time to a session's starting date, instead of basing it upon each individual class section's first meeting date. Secondly, each session's end date was often later than many of its classes' respective last meeting dates. Hence, these small disparities may occur at the session's beginning or its end; or possibly both, with a course's later start, for example, potentially cancelling out its earlier final-exam date. In 
fact, for the institution in this study, the date of a class's final exam was dependent not only upon the day(s) of the week the class met, but also varied based upon time-of-day blocks within the given day(s) of the week. For example, a Monday-Wednesday-Friday (MWF) 9:00-9:50am 16-week (Session I) class may have had its final exam on Wednesday, December 12, which would have been a full six days prior to the Session I end date of December 18. Meanwhile, a 16-week MWF 10:00-10:50am class may not have had its institutionally-assigned final-exam date until five days later, on Monday, December 17. Consequently, these small differences were deemed trivial and, moreover, unwieldy.

In contrast, a far more important concern about the operational definition of the survival analysis's starting time arose: how might the large differences in starting dates for the three sessions (i.e., August 22 for Session I, September 19 for Session III, and October 18 for Session IV) adversely affect the interpretability of the analysis? Although it is neither problematic nor atypical for a survival analysis to assign varying starting times to different subjects (e.g., birthdate is a common starting time in many medical studies), the variation in time duration among the three course session types was a concern in terms of complicating the interpretation of student attrition rates. In short, the following question needed to be addressed: how should a student withdrawal in the fourth week of a Session IV (8-week) mini-term course be equated to a student withdrawal in the fourth week of a Session III (12-week) mini-term course and, in turn, equated to a student withdrawal in the fourth week of a Session I (16-week) full-semester course, so that the interpretation of the analysis is not distorted? The solution was to adjust the time metric. 
The time metric's definition and related considerations. With survival-analysis data, Singer and Willett (2003) stress that, "Time should be recorded in the smallest possible units relevant to the process under study" (p. 313). Thus, because each student's course withdrawal (W) grade is accompanied by the calendar date of the withdrawal, it made sense to operationally define the time metric as the number of days elapsed between the session's starting date and the recorded withdrawal date. Defining the time metric in this way, however, required that three data-set-construction problems be remedied.

First, the large calendar-day differences in session durations (as previously detailed in Table 1) demanded a type of proportionality adjustment, so that withdrawal times for mini-term Session III and IV students could be compared accurately to those of full-semester Session I enrollees. Providing an example might better clarify this issue and its gravity. Suppose one student in a Session I (16-week) course withdrew on October 30, 2001, which was the student withdrawal deadline for all Session I classes. That student's survival time would have been 69 days; that is, 69 would have been the number of days elapsed between the official start of Session I (August 22) and the student's withdrawal (on October 30). However, 69 days exceeds the entire 61-day duration of the 8-week (Session IV) class. Therefore, a Session I student who withdrew at or near the midsemester withdrawal deadline would have a survival time that exceeded the rightcensored time of a student who completed the Session IV class.

To correct for this flaw, the survival times for the students in the 20 Session III (90-day) classes and the one Session IV (61-day) class were each appropriately prorated, so that they could be compared accurately to the survival times of Session I (118-day) 
students. Specifically, the raw Session III and Session IV survival times were multiplied by ratios of $118 / 90$ and 118/61, respectively.

The second of the three time-metric challenges that arose was discovered after performing a preliminary cross-tabulation analysis of student withdrawal dates. This revealed three students whose $\mathrm{W}$ grades were accompanied by withdrawal dates that were well after the official (December 18, 2001) semester-end date. In fact, all three of these students had been assigned 2002 withdrawal dates; specifically, 1/17/02, 3/13/02, and $8 / 6 / 02$. It is likely that these were post-semester changes-of-grade that either were submitted by the instructor or were the outcome of a grade-appeal process. No matter the reason, these three withdrawal dates were changed in the survival-analysis data set to 12/18/01, the official end-date of the Fall-2001 semester. This increased the number of cases with 12/18/01 withdrawal dates from 132 to 135 .

The third of the three major time-metric challenges pertained to the fact that $22.7 \%(n=135)$ of the 594 students who experienced the survival event (i.e., a course grade of W) had a recorded withdrawal date of December 18, which was the last official day of the Fall-2001 semester. Hence, all of these 135 students had survival times of 118 days that equaled the maximum possible duration of this survival analysis's tracking period, due to the timespan of the full-semester (Session I) classes being 118 days in length. This begged the question: was the dichotomous coding of the survival event $(1=\mathrm{W}$ grade; $0=$ not $\mathrm{W}$ grade $)$ a sufficient way to avoid commingling these subjects with the remaining 2,690 subjects who did not experience the survival event (i.e., were assigned a course grade other than W), yet paradoxically were incapable of having an actual survival time in excess of the 118-day duration of the semester? 
Although this is typically a non-issue with survival analysis methods due to the use of appropriate right-censoring techniques to account for subjects who may experience the event after the study's time period, it was an initial concern with this data set due to the fact that the $12 / 18 / 01$ end to this research question's time period also was the end of the actual course. Therefore, unlike most studies that apply survival-analysis methods, it was not possible for a subject to experience the survival event of interest at some future unknown date after the tracking period.

Three ways of addressing this concern were considered and, moreover, were even investigated with the aid of SPSS: (a) omitting survival times for the 2,690 nonwithdrawal students who did not experience the survival event; (b) affixing a survival time in excess of 118 days to those non-withdrawal students (such as 119 days or 125 days) in order to better distinguish them from those who received a $\mathrm{W}$ grade with an endof-term last-date-of-attendance of 12/18/02 (i.e., an 118-day survival time) ; and (c) assigning the 2,690 non-withdrawal cases survival times of 118 days, the full and actual duration of the tracking period.

The first option was by far the worst of the three, for doing so would have excluded from the analysis a vitally relevant subset of the Fall-2001 MAT0024 student cohort: all students who were not assigned a withdrawal (W) grade. Such an exclusion would have prevented any comparative analyses between course completers and noncompleters. Additionally, it would have resulted in an underestimation of average survival time. As Singer and Willett (2003) state, “... data from both the censored and the uncensored cases must be incorporated simultaneously in the analysis.... Censored cased 
must not be excluded, even though we [may] not know when... [or even if] they will ultimately experience the target event" (p. 322)

The second approach (i.e., assigning course completers a survival time of 119 or more to better distinguish them from non-completers with survival times of 118 days) would have inflated mean and median survival times, albeit marginally. Also, adding a day or, for that matter, an entire week to the survival times of completers would have been an arbitrarily-based decision, devoid of a cogent rationale. Furthermore, attempting it proved to the researcher that doing so in no way benefitted the analysis. Therefore, the 2,690 non-withdrawal subjects were assigned right-censored survival times of 118 days, the exact duration of the Fall-2001 semester and, hence, the MAT0024 course tracking period.

\section{DTSA Considerations for Research Question 3}

Whereas the second research question encompassed a survival-analysis examination of a one-semester, 118-day time frame, the third research question spanned a lengthier two-year period. This question asked: Across the sequence of communitycollege algebra courses, is there a longitudinal difference in the time to complete at least one general-education college-level mathematics course between students who satisfied the prerequisite algebra course requirements by completing one or more algebra courses scheduled in a one-day-per-week, longer-duration format and students whose prerequisite algebra course requirements were entirely fulfilled in courses that had medium-duration (twice weekly) or shorter-duration (thrice weekly) class meetings?

The definitions of the survival-analysis core constructs applicable to this question — target event, time metric, and initial starting time — were all different than 
those applicable to the second research question. For this third research question, the target event (also known as the hazard) was operationalized as the cohort student's first general-education level mathematics course completed successfully, subsequent to the student's enrollment in an MAT0024 Elementary Algebra course during the Fall-2001 semester. So, it is important to keep in mind that - contrary to the harmful connotation usually implied by the word hazard - this survival analysis's hazard event is a healthy, positive outcome: a passing grade in a college-level mathematics course.

What exactly is a general-education level mathematics course? Although Florida colleges and universities have some latitude with course titles, course prefixes and course numbering for general-education mathematics courses (GEMCs) are consistent statewide. GEMCs include: MAC1105 College Algebra, MGF1106 Mathematics for Liberal Arts I, MGF1107 Mathematics for Liberal Arts II, STA2023 Statistics, as well as an assortment of higher-level mathematics courses. MAC1105, MGF1106/1107, and STA2023 all share a common course prerequisite of a grade of "C" or higher in MAT1033 Intermediate Algebra. MAT1033's course prerequisite was MAT0024 Elementary Algebra. Unlike the typical Fall-2001 MAT0024 cohort student who had low placement-test scores that demanded completion of one or both developmental-level mathematics courses (i.e., MAT0024 and MAT0012 Pre-Algebra), some students placed directly into MAT1033 or even a higher-level mathematics course.

The time metric was defined as the number of semesters elapsed since the conclusion of the Fall-2001 semester, partitioned into half semester sub-intervals. Within each academic year, there were three semesters: fall, winter, and summer. Whereas the fall and winter terms were each approximately 16 weeks in duration, the summer 
semester was a 12-week period. To enhance the metric's precision, semesters were partitioned into half semester sub-intervals due primarily to many summer mathematics course offerings having been scheduled in six-week mini-sessions. Consequently, it was not uncommon in the summer for a student to attempt two mathematics courses in a consecutive manner; namely, one course during the first six-week mini-session and another course during the second six-week mini-session.

Meanwhile, other summer mathematics courses spanned the full 12-week semester. To distinguish between a student who completed, for example, two mathematics courses in a consecutive manner during the 12-week summer term, it was important to partition the time metric into half-semester intervals.

So, for example, students who successfully completed their first generaleducation mathematics course in the first 6-week mini-session of the Summer-2003 semester would have had a survival-event times of 4.5; that is, four and a half semesters after the Fall-2001 MAT0024 course, these students passed their first general-education mathematics course. It should be noted that this study's longitudinal (Fall-2001 through Summer-2003) data set included no fall-semester nor winter-semester general-education mathematics courses that were scheduled in half-semester-duration mini-sessions. In fact, only a total of seven developmental-level (MAT0012/0024) courses were scheduled in that way, during the Winter-2002, Fall-2002, and Winter-2003 semesters.

The initial starting time was defined as the conclusion of the Fall-2001student's Fall-2001 MAT0024 Elementary Algebra course. In the case of all of the Fall-2001 MAT0024 class sections $(N=120)$, the reader is reminded that, as the previously shown Table 1 illustrated, this conclusion date was essentially the same: December 18, 2001. 
Therefore, because the time metric for this question was not measured in days, one may choose to think of the survival analysis's starting time as the beginning of the Winter2002 semester; namely, January of 2002.

\section{Limitations}

There were several important limitations to this research methodology. First, due to the ex post facto design of this study and the fact that seat time is not a treatment that can be randomized, causality-type conclusions (e.g., three-hour, one-day-per-week classes cause lower course success rates) could not be inferred nor ascertained from the findings of this study. Second, other than a common course outline with stated objectives and a standardized state-mandated final exam in the MAT0024 Elementary Algebra course, the large multi-campus community college selected is one that does not have a high degree of consistency/uniformity in course-grading standards. Instructors have considerable authority in determining their course testing/grading policies and their pedagogical methods (e.g., pure lecture versus a wider variety of classroom activities), which is an unmeasured variable of potential concern. Third, there are other potentially confounding variables which future researchers may wish to consider yet this research methodology did not include; for instance: the number of hours worked per week by the student; the highest level of mathematics coursework completed prior to enrolling in a postsecondary institution and how recently it was completed; the extent, if at all, to which the student utilized ancillary academic support services and the type(s) of services utilized (e.g., online/multimedia course management systems and on-campus learningresource-center tutoring services); the amount and type of homework assigned/graded by 
the instructor and its weight, if any, in the computation of student grades; and the number and type(s) of tests and/or quizzes given throughout the course.

\section{Summary}

After restating the purpose of this research dissertation study, this chapter addressed, in order, eight methodological matters: (a) the study's research questions and hypotheses, (b) its research design, (c) the defined population and chosen sample, (d) the setting and extent of interaction with the subjects in its sample, (e) the data-collection procedure, (f) the variables, (g) the data-analysis techniques employed, and (h) the study's methodology-related limitations.

Chapter 4 details the quantitative analyses performed and their results. 


\section{CHAPTER IV}

\section{ANALYSES \& FINDINGS}

This chapter reports the details and results of the multilevel data-analysis methods employed. The chapter begins by providing demographic summaries and descriptive statistics pertaining to the Fall-2001 MAT0024 cohort's two levels of variables; that is, student-level (Level-1) variables followed by similar summaries for the course-section level (Level-2) variables. Within this first portion of the chapter, the reader will find not only a wide-ranging summary of aggregated demographic and academic-related variables, but also an examination of many of these variables disaggregated by the two variables of primary interest: the predictor variable (seat time) and the first research question's outcome variable (success in Fall-2001 MAT0024 Elementary Algebra course). Then, in the order of the research questions, the remainder of the chapter provides the study's findings in response to each of the three research questions and their respective hypotheses.

\section{Research Questions and Hypotheses}

For ease of reference, the reader is reminded of this study's research questions and their corresponding research hypotheses:

1. In community-college developmental-level algebra courses, is there a difference in student success for students enrolled in classes that meet one day per week in longer-duration class meetings versus for those enrolled in medium-duration (twice weekly) class meetings versus for those enrolled in shorter-duration (thrice weekly) class meetings? 
2. In community-college developmental-level algebra courses, is discrete survival time, as measured by date of withdrawal from the course, related to the number of class meetings per week and the duration of each meeting?

3. Across the sequence of community-college algebra courses, is there a longitudinal difference in the time to complete at least one general-education college-level mathematics course between students who satisfied the prerequisite algebra course requirements by completing one or more algebra courses scheduled in a one-day-per-week, longer-duration format and students whose prerequisite algebra course requirements were entirely fulfilled in courses that had medium-duration (twice weekly) or shorter-duration (thrice weekly) class meetings?

Based upon this dissertation author's literature-review findings, the three research hypotheses for this study were:

$\mathrm{H}_{1}$ : There is a difference in student success for students enrolled in classes that meet one day per week in longer-duration class meetings versus for those enrolled in medium-duration (twice weekly) class meetings versus for those enrolled in shorterduration (thrice weekly) class meetings.

$\mathrm{H}_{2}$ : Discrete survival time in community-college developmental-level algebra courses, as measured by date of withdrawal from the course, is related to the number of class meetings per week and the length of each meeting.

$\mathrm{H}_{3}$ : Across the sequence of community-college algebra courses, there is a longitudinal difference in the time to complete at least one general-education collegelevel mathematics courses between students who satisfied the prerequisite algebra course 
requirements by completing one or more algebra courses scheduled in a one-day-perweek, longer-duration format and students whose prerequisite algebra course requirements were entirely fulfilled in courses that had medium-duration (twice weekly) or shorter-duration (thrice weekly) class meetings.

\section{Demographic Attributes of Sample and Descriptive Statistics}

At their essence, all three of this study's research questions are inquiries into the academic outcomes - be it in a particular developmental-algebra course, either in a particular semester or longitudinally (over a six-semester tracking period) through the sequence of general-education mathematics courses-for one particular population cohort of students: all Fall-2001 Elementary Algebra (MAT0024) students at a large, urban, public, multi-campus South Florida community college $(N=3,284)$.

Therefore, in light of the assorted differences that often exist between student enrollees in the varying course-scheduling options (e.g., age and course-load differences between daytime students versus evening students, and race/ethnicity differences among campus localities), it was important in this multilevel study to acquire and consider a wide array of demographic (covariate) variables for the student subjects. To decide which demographic variables to include in the researcher's data-collection request, the recommendations of various methodologists were reviewed. Among them, for example, were Bini, Monari, Piccolo, and Salmaso (2009). Another was Andreu (2002), who utilized Tinto's and Bean's models of retention to compile and define more than 20 independent variables for community college institutional researchers to consider when examining student retention. Additionally, this researcher's selection of covariates was guided by the multivariate analyses and findings of comprehensive community-college 
persistence-type studies. Of particular interest were multivariate studies that utilized multi-institutional data sets and/or longitudinally tracked a student cohort (e.g., Fike \& Fike, 2008; Moosai, Walker, \& Floyd, 2011).

\section{Aggregated Demographic Summary}

In an aggregated manner, Table 2 summarizes the following five demographic attributes of the Fall-2001 MAT0024 student cohort: sex, ethnicity (dichotomously categorized as Hispanic versus Non-Hispanic), race, birthplace (i.e., country of origin dichotomously recoded by the researcher as U.S. native-born versus not), and immigration status at the start of the Fall-2001 semester (after the researcher grouped the 18 distinct immigration-status codes in the raw data set into 5 main classifications). 
Table 2

Frequency Table of MAT0024 Student Cohort Demography

\begin{tabular}{|c|c|c|c|}
\hline & & $f$ & $\%$ \\
\hline \multirow[t]{5}{*}{ Sex } & Female & 2,080 & 63.3 \\
\hline & Male & 1,200 & 36.5 \\
\hline & Reported & 3,280 & 99.9 \\
\hline & Unreported & 4 & .1 \\
\hline & Total & 3,284 & 100.0 \\
\hline \multirow[t]{5}{*}{ Ethnicity $^{\mathrm{a}}$} & Hispanic & 820 & 25.0 \\
\hline & Non-Hispanic & 233 & 7.1 \\
\hline & Reported & 1,053 & 32.1 \\
\hline & Unreported & 2,231 & 67.9 \\
\hline & Total & 3,284 & 100.0 \\
\hline \multirow[t]{8}{*}{$\operatorname{Race}^{\mathrm{a}}$} & Asian & 83 & 2.5 \\
\hline & Black & 1,119 & 34.1 \\
\hline & Native American & 15 & .5 \\
\hline & Pacific Islander & 0 & .0 \\
\hline & White & 1,208 & 36.8 \\
\hline & Reported & 2,425 & 73.8 \\
\hline & Unreported & 859 & 26.2 \\
\hline & Total & 3,284 & 100.0 \\
\hline \multirow[t]{5}{*}{ Birthplace } & Foreign Born & 1,013 & 30.8 \\
\hline & U.S. Born & 2,265 & 69.0 \\
\hline & Reported & 3,278 & 99.8 \\
\hline & Unreported & 6 & .2 \\
\hline & Total & 3,284 & 100.0 \\
\hline \multirow[t]{8}{*}{ Immigration Status } & F-1 Student Visa & 73 & 2.2 \\
\hline & Permanent Resident Alien & 570 & 17.4 \\
\hline & Other Documented Type & 81 & 2.5 \\
\hline & Undocumented & 3 & .1 \\
\hline & U.S. Citizen & 2,557 & 77.9 \\
\hline & Reported & 3,284 & 100.0 \\
\hline & Unreported & 0 & .0 \\
\hline & Total & 3,284 & 100.0 \\
\hline
\end{tabular}

${ }^{\mathrm{a}}$ The race/ethnicity types are based upon the categories developed in 1997 by the White House's Office of Management Budget (OMB). These categories “.... are used to describe groups to which individuals belong, identify with, or belong in the eyes of the community... [They] do not denote scientific definitions of anthropological origins. The designations are used to categorize U.S. citizens, resident aliens, and other eligible non-citizens" (Knapp, Kelly-Reid, \& Ginder, 2012, p. B-5). 
As Table 2 shows, the 3,284 students in the MAT0024 Fall-2001 cohort were predominately female (63.3\%). The cohort's proportion of women is slightly higher than — but is still proportionally comparable to - the institution-wide and state-wide female representation levels (61.9\% and 59.8\%, respectively) for the same time period as this dissertation study, as reported by the Florida Department of Education (Florida Division of Community Colleges, 2002).

On average, slightly more than 3 out of every 10 cohort students $(30.8 \%)$ were foreign born $(n=1,013)$. Based upon educational-system and/or language-custom differences, the term foreign born was operationally defined to include - in addition, of course, to students who were born in other nations - students who were born in any of the United States' 14 territories. So, 39 (or 3.8\%) of the 1,013 students classified as foreignborn were born in U.S. territories; specifically, Puerto Rico $(n=32)$ and the U.S. Virgin Islands $(n=7)$. In all, these 1,013 foreign born students represented 79 different nations and the two aforementioned U.S. territories. Nearly three-fourths $(72.6 \%)$ of these foreign-born students were born in one of the following 11 most often represented nations (in descending frequency order): Jamaica 25.0\% $(n=253)$, Haiti $13.4 \%(n=$ 136), Colombia 10.0\% $(n=101)$, the Dominican Republic 3.4\% $(n=34)$, Puerto Rico $3.2 \%(n=32)$, Peru 3.1\% $(n=31)$, Trinidad \& Tobago $3.1 \%(n=31)$, Venezuela $3.1 \%(n$ $=31)$, Bahamas 2.9\% $(n=29)$, Brazil 2.7\% $(n=27)$, and Cuba 2.7\% $(n=27)$.

As for immigration/residency status, only $4.7 \%$ of the cohort students were neither U.S. Citizens nor Permanent Resident Aliens. The $2.5 \%(n=81)$ within what the researcher termed the Other Documented Type category is comprised of the researcher's own subtotaling of 14 different documented statuses, ranging from asylees to tourists. 
There are two striking differences between the variables of birthplace (i.e., country of origin) and immigration status versus those of ethnicity and race: response rate and internal consistency.

First, the response rate for birthplace and immigration status $(99.8 \%$ and $100 \%$, respectively) was markedly higher than that of race and ethnicity $(73.8 \%$ and $32.1 \%$, respectively). This was largely attributable to the fact that the admissions process requires legal documentation to verify each student's immigration status, which in turn is crosschecked against the student's self-reported country of origin. Due to the documentation that is required and verified by college officials during the admissions process, there were only six student subjects with missing values for birthplace and none for immigration status.

In contrast, the variables of ethnicity and race are optional-response items contained within the college's admissions application. Neither entails any institutional verification process. Consequently, many students choose - for a variety of reasons - to not answer. In the case of this study's Fall-2001 MAT0024 cohort, more than two-thirds (67.9\%) did not identify their ethnicity, which was subdivided into two mutuallyexclusive categories: Hispanic or Non-Hispanic. More than one-fourth (26.2\%) of the cohort did not identify their race, which was partitioned into five categories (Asian, Black/African-American, Native American, Pacific Islander, White/Caucasian) with at most one response per subject permitted.

The second striking difference between the variables of birthplace (i.e., country of origin) and immigration status versus those of ethnicity and race pertains to their respective degrees of internal consistency. With regard to the variables of race and 
ethnicity, it should be noted that, during the college's admissions process, the students self-identify themselves according to their predominating racial/ethnic self-perceptions at the time. A multiracial student would either have to select one racial category or opt to not respond at all. Moreover, as various ethnic-identity development theories posit, the self-perceptions of multiracial/multiethnic students—-particularly for those of color-may very well end up being different several semesters later, as a result of the socialization experiences induced by the collegiate experience and (for traditional-age college students) the transition from adolescence to adulthood (Pahl \& Way, 2006). Since answering these application questions is optional, many students choose - for a variety of reasons - to not answer. Because of the way in which this demographic data were obtained by the institution and the fact that there was a high rate of unreported racial/ethnic classifications, it is likely that these covariate measures lack internal consistency and, hence, reliability.

Despite the cohort's high rates of unreported values for ethnicity and (albeit it to a lesser extent) race, the diversity of the institution is, nonetheless, made apparent by the fact that: (i) $25.0 \%$ of the 3,284 cohort students identified themselves as Hispanic, and (ii) the ratio of White-to-Black students was approximately equal $(1,208: 1,119)$ among the $2,425(73.8 \%)$ of the cohort students who reported their racial classification. Based upon the Florida Department of Education's annual Fact Book report (Florida Division of Community Colleges, 2002), the institution's college-wide racial/ethnic demographics for this same Fall-2001 time period are comparable to that of the study's cohort, although the college-wide percentage of Non-Hispanic White students is a bit higher (40.3\%) than that of the cohort while the proportions of Non-Hispanic Black and Hispanic students are 
somewhat lower $(25.7 \%$ and $21.2 \%$, respectively). Additionally, the cohort's race/ethnicity proportions are not only consistent with the institution's college-wide demography; they are also consistent with the college's designation as a Postsecondary Hispanic-Serving Institution (HSI) and as a Postsecondary Minority Institution, as defined by the U.S. Higher Education Act (Hispanic Association of Colleges \& Universities, 2012; U.S. Department of Education, 2010a, 2010b).

\section{Demography Disaggregated by IV of Primary Interest (Seat Time)}

To preliminarily assess (prior to addressing the research questions) whether demographic-variable differences existed among the three levels of seat-time duration, the aforementioned five demographic variables were accordingly disaggregated. Table 3 displays that disaggregation in terms of both raw and relative frequencies. 
Table 3

MAT0024 Cohort Demographics Disaggregated by Seat-Time Level

\begin{tabular}{|c|c|c|c|c|c|c|c|}
\hline \multirow{3}{*}{\multicolumn{2}{|c|}{ Demographic }} & \multicolumn{5}{|c|}{ Seat Time } & \multirow[b]{3}{*}{ Total } \\
\hline & & \multicolumn{2}{|c|}{ Short } & \multicolumn{2}{|c|}{ Medium } & Long & \\
\hline & & $n$ & $(\%)$ & $n$ & $(\%)$ & $\begin{array}{ll}n & (\%) \\
\end{array}$ & \\
\hline \multirow{2}{*}{ Sex } & Female & 678 & $(60.7)$ & 1,050 & $(63.3)$ & $352(70.0)$ & 2,080 \\
\hline & Male & 439 & (39.3) & 610 & (36.7) & $151(30.0)$ & 1,200 \\
\hline \multirow{3}{*}{ Ethnicity } & Hispanic & 329 & (29.4) & 396 & $(23.8)$ & $95(18.8)$ & 820 \\
\hline & Non-Hispanic & 79 & (7.1) & 120 & $(7.2)$ & $34 \quad(6.7)$ & 233 \\
\hline & Unreported & 710 & $(63.5)$ & 1,146 & $(69.0)$ & $375(74.4)$ & 2,231 \\
\hline \multirow{5}{*}{ Race } & Asian & 34 & $(3.0)$ & 36 & $(2.2)$ & $13 \quad(2.6)$ & 83 \\
\hline & Black & 369 & $(33.0)$ & 562 & (33.8) & $188(37.3)$ & 1,119 \\
\hline & Native & 2 & $(0.2)$ & 11 & $(0.7)$ & $2(0.4)$ & 15 \\
\hline & White & 377 & $(33.7)$ & 629 & $(37.8)$ & $202(40.1)$ & 1,208 \\
\hline & Unreported & 336 & $30.1)$ & 424 & $(25.5)$ & $99(19.6)$ & 859 \\
\hline \multirow{2}{*}{ Birthplace } & Foreign Born & 342 & (30.6) & 506 & $(30.5)$ & $165(32.8)$ & 1,013 \\
\hline & U.S. Born & 774 & (69.4) & 1,153 & $(69.5)$ & $338(67.2)$ & 2,265 \\
\hline \multirow{5}{*}{$\begin{array}{l}\text { Immigration } \\
\text { Status }\end{array}$} & F-1 Student & 36 & (3.2) & 34 & $(2.0)$ & $3 \quad(0.6)$ & 73 \\
\hline & Perm. Res. & 178 & $(15.9)$ & 286 & $(17.2)$ & $106(21.0)$ & 570 \\
\hline & Other Doc. & 25 & $(2.2)$ & 44 & $(2.6)$ & $12 \quad(2.4)$ & 81 \\
\hline & Undocumented & 1 & $(0.1)$ & 1 & $(0.1)$ & $1 \quad(0.2)$ & 3 \\
\hline & U.S. Citizen & 878 & $(78.5)$ & 1,297 & $(78.0)$ & $382(75.8)$ & 2,557 \\
\hline
\end{tabular}

Using SPSS Version 20’s Crosstabs procedure, a series of chi-square tests for homogeneity of proportions was performed to ascertain significant relative-frequency differences, if any, between each of these student-demographic variables and the three levels of seat time. Table 4 provides the results. For any test that yielded a significant $\chi^{2}$ test-statistic value (at the $\alpha=.05$ significance level), the absolute value of each cell's standardized residuals was compared against 2.00 in order to identify any major contributors to the $\chi^{2}$ value, as recommended by Hinkle, Wiersma, and Jurs (2003). Appropriate correlation-coefficient measures, such as Cramer's V and the less-often-used Rank-Biserial applicable to nominal-to-ordinal bivariate correlations, were computed and 
considered, but they were ultimately deemed unnecessary at this preliminary stage of the data-analysis process.

Significant differences in gender proportionality $\left(\chi^{2}=12.917, p=.002\right)$ among the three seat-time levels were detected. Upon further inspection of each cell's standardized residuals, this difference was determined to be most attributable to a larger female-to-male imbalance in long seat-time classes $(70.0 \%$ to $30.0 \%)$ than was the case in short $(60.7 \%$ to $39.3 \%)$ and medium $(63.3 \%$ to $36.7 \%)$ classes. The only standardized residual with an absolute value of at least 2.0 found was that of the long seat-time male students (specifically, -2.4).

Table 4

Homogeneity-of-Proportion Tests for Student Demographics * Seat Time

\begin{tabular}{|c|c|c|c|c|}
\hline & & Value & $d f$ & Asymp. Sig. (2-sided) \\
\hline \multirow{2}{*}{$\begin{array}{c}\text { Sex }{ }^{*} \mathrm{ST} \\
(n=3,280)\end{array}$} & Pearson Chi-Square & $12.917^{\mathrm{a}}$ & 2 & .002 \\
\hline & Likelihood Ratio & 13.160 & 2 & .001 \\
\hline \multirow{2}{*}{$\begin{array}{c}\text { Ethnicity* } \mathrm{ST}^{\mathrm{a}} \\
(n=1,053)\end{array}$} & Pearson Chi-Square & $3.530^{\mathrm{a}}$ & 2 & .171 \\
\hline & Likelihood Ratio & 3.528 & 2 & .171 \\
\hline \multirow{2}{*}{$\begin{array}{c}\text { Race*ST }^{\mathrm{a}} \\
(n=2,425)\end{array}$} & Pearson Chi-Square & 7.183 & 6 & .304 \\
\hline & Likelihood Ratio & 7.393 & 6 & .286 \\
\hline \multirow{2}{*}{$\begin{array}{l}\text { Birthplace*ST } \\
\quad(n=3,278)\end{array}$} & Pearson Chi-Square & 1.011 & 2 & .603 \\
\hline & Likelihood Ratio & 1.002 & 2 & .606 \\
\hline \multirow{2}{*}{$\begin{array}{l}\text { Immig. Status* } \mathrm{ST} \\
\quad(n=3,284)\end{array}$} & Pearson Chi-Square & 18.146 & 8 & .020 \\
\hline & Likelihood Ratio & 19.689 & 8 & .012 \\
\hline
\end{tabular}

Because of the high unreported value rates for race and ethnicity, the missing-value cases were treated in two different ways. First, in the above analysis, the unreported values were left uncoded (i.e., as empty cells). As shown in Table 4, this approach resulted in neither race nor ethnicity evidencing significant differences in proportionality across seat- 
time levels. Second, the alternative strategy taken was to assign the unreported values a code, just like all of the reported values for these nominal variables were given. The rationale for doing so was to assess whether the proportions of unreported values, when included with the reported values, altered the chi-square results.

When the test was performed this way, the resulting test-statistic values changed significantly; for instance, in the case of the race variable, $\chi^{2}=37.220, d f=8, p<.001$. The standardized residuals in this test revealed that the only major differences in observed-versus-expected frequencies were attributable to the unreported cases. Specifically, the unreported cases for the short seat-time level were proportionally overrepresented (with a standardized residual value of +2.5 ), while the unreported cases for the long seat-time level were under-represented (with a standardized residual value of 2.9). The medium seat-time level contained no significant proportional differences, neither for the unreported cases nor for any of the other race values.

Similarly, when the homogeneity of proportions for the ethnicity variable were analyzed in this manner, the result was $\chi^{2}=24.040, d f=4, p<.001$. However, the absolute values of the standardized residuals all were below 2.0, with none above 1.8.

The next demographic variable analyzed was student age. In the aggregate, the mean age of the Fall-2001 MAT0024 student cohort was 23.4 years, with a standard deviation value of 7.26. At the session start date of their Fall-2001 MAT0024 class, the youngest student was 16 , and the oldest was 62 . As one would expect with collegestudent ages, the distribution shape was positively skewed, as evidenced by its skewness coefficient value (2.048) and related plots (e.g., box-and-whisker and stem-and-leaf). 
Table 5 summarizes student-age statistics and distribution shape for each of the three levels of seat time. Results show that older students tend to enroll in long and medium seat-time class sections rather than in short ones; in contrast, younger students register more frequently in short seat-time classes. This was not at all surprising, because all of the short seat-time classes $(n=40)$ in this study's sample were daytime offerings, while all of the long seat-time classes $(n=20)$ were evening or weekend sections.

Daytime college students are, on average, younger than evening-weekend students, who tend to be older and employed during the day.

Table 5

Homogeneity-of-Proportion Tests for Student Demographics * Seat Time

\begin{tabular}{lcccccrr}
\hline \begin{tabular}{l} 
Seat-Time \\
\multicolumn{1}{c}{ Level }
\end{tabular} & $M$ & $95 \% \mathrm{CI}$ & $\mathrm{Mdn}$ & $S D$ & {$[$ Min,Max] } & Skewness & Kurtosis \\
\hline Short & 21.49 & $(21.15,21.83)$ & 19.51 & 5.73 & {$[16.48,62.77]$} & 3.203 & 12.179 \\
Medium & 23.26 & $(22.92,23.60)$ & 20.38 & 7.03 & {$[16.12,60.39]$} & 2.106 & 4.496 \\
Long & 28.34 & $(27.58,29.10)$ & 25.97 & 8.68 & {$[16.90,59.42]$} & 1.023 & 0.438 \\
\hline
\end{tabular}

Due to the lack of homogeneity in variances among these three groups and the lack of normality in the underlying (aggregated) distribution, neither a one-way ANOVA nor a pairwise student $t$-test comparison of ages was appropriate. However, the fact that none of the $95 \%$ confidence-interval (CI) estimates overlapped — and, most notably, how much comparatively higher the long seat-time level's CI is - indicates substantial group differences in average age.

\section{Disaggregation of Academic-Related Background Covariates}

A variety of Level-1 academic-background variables were disaggregated in two ways: by the primary IV (seat-time), and then by the first research question's dichotomous DV (success in the Fall-2001 MAT0024 course). 
Table 6 summarizes the relative frequency distribution of nine academic-related covariates. Among these nine, student enrollment status (i.e., full-time versus part-time) displayed the most striking, statistically significant difference between the three seat-time groups $\left(\chi^{2}=262.775, d f=2, p<.001\right)$. While full-time students exceeded part-time students in the short seat-time classes by nearly a 3:2 ratio, the opposite was true within the other two seat-time groups; that is, part-time exceeded full-time enrollees by margins in excess of 3:2 and 4:1 for the medium and long seat-time groups, respectively. This is consistent with the between-group differences in student ages (as was shown in Table 5), since full-time enrollees tend to be younger, traditional-age students, whereas older, nontraditional-age students typically register for part-time course loads. 
Table 6

Frequency Distribution of Academic-Related Covariates

\begin{tabular}{|c|c|c|c|c|c|c|c|c|c|}
\hline \multirow{3}{*}{\multicolumn{2}{|c|}{$\begin{array}{l}\text { Academic } \\
\text { Covariates }\end{array}$}} & \multicolumn{6}{|c|}{ Seat Time } & \multirow{2}{*}{\multicolumn{2}{|c|}{ Total }} \\
\hline & & \multicolumn{2}{|c|}{ Short } & \multicolumn{2}{|c|}{ Medium } & \multicolumn{2}{|c|}{ Long } & & \\
\hline & & $n$ & $(\%)$ & $n$ & $(\%)$ & $n$ & $(\%)$ & $n$ & $(\%)$ \\
\hline \multirow{2}{*}{$\begin{array}{l}\text { Full-Time } \\
\text { Enrollee }^{\mathrm{a}} \\
\end{array}$} & Yes & 658 & $(58.9)$ & 660 & $(39.7)$ & 85 & $(16.9)$ & 1,403 & (42.7) \\
\hline & $\mathrm{No}^{\mathrm{a}}$ & 460 & $(41.1)$ & 1,002 & $(60.3)$ & 419 & $(83.1)$ & 1,881 & $(57.3)$ \\
\hline \multirow{2}{*}{ Financial Aid ${ }^{b}$} & Yes & 410 & $(36.7)$ & 577 & $(34.7)$ & 163 & (32.3) & 1,150 & $(35.0)$ \\
\hline & No & 708 & $(63.3)$ & 1,085 & $(65.3)$ & 341 & $(67.7)$ & 2,134 & (65.0) \\
\hline \multirow{2}{*}{$\begin{array}{l}\text { Verified } \\
\text { Disability } \\
\end{array}$} & Yes & 27 & (2.4) & 21 & $(1.3)$ & 1 & $(0.2)$ & 49 & $(1.5)$ \\
\hline & No & 1,091 & (97.6) & 1,641 & $(98.7)$ & 503 & $(99.8)$ & 3,235 & (98.5) \\
\hline \multicolumn{2}{|c|}{ SLS attempted Yes } & 250 & $\overline{(22.4)}$ & 324 & $(19.5)$ & 96 & $(19.0)$ & 670 & $\overline{(20.4)}$ \\
\hline previously & No & 868 & $(77.6)$ & 1,338 & $(80.5)$ & 408 & $(81.0)$ & 2,614 & (79.6) \\
\hline \multirow{2}{*}{$\begin{array}{l}\text { SLS passed } \\
\text { previously }\end{array}$} & Yes & 221 & $(19.8)$ & 271 & $(16.3)$ & 87 & $(17.3)$ & 579 & $(17.6)$ \\
\hline & No & 897 & $(80.2)$ & 1,391 & $(83.7)$ & 417 & $(82.7)$ & 2,705 & $(82.4)$ \\
\hline \multirow{3}{*}{$\begin{array}{l}\text { Dev.-Level } \\
\text { Math } \\
\text { Placement }\end{array}$} & Lowest & 256 & (22.9) & 372 & $(22.4)$ & 157 & $(31.2)$ & 785 & (23.9) \\
\hline & Highest & 758 & $(67.8)$ & 1,056 & $(63.5)$ & 243 & $(48.2)$ & 2,057 & $(62.6)$ \\
\hline & Neither & 104 & $(9.3)$ & 234 & $(14.1)$ & 104 & (20.6) & 442 & (13.5) \\
\hline \multirow{3}{*}{$\begin{array}{l}\text { Reading } \\
\text { Placement } \\
\text { Status }\end{array}$} & Prep & 642 & (57.4) & 861 & $(51.8)$ & 188 & $(37.3)$ & 1,691 & $\overline{(51.5)}$ \\
\hline & Coll-Ready & 396 & $(35.4)$ & 591 & $(35.6)$ & 200 & (39.7) & 1,187 & $(36.1)$ \\
\hline & Unknown & 80 & $(7.2)$ & 210 & $(12.6)$ & 116 & $(23.0)$ & 406 & (12.4) \\
\hline \multirow{3}{*}{$\begin{array}{l}\text { Writing } \\
\text { Placement } \\
\text { Status }\end{array}$} & Prep & 453 & $(40.5)$ & 602 & $(36.2)$ & 147 & $(29.2)$ & 1,202 & $(36.6)$ \\
\hline & $\begin{array}{l}\text { College- } \\
\text { Ready }\end{array}$ & 541 & $(48.4)$ & 798 & $(48.0)$ & 229 & $(45.4)$ & 1,568 & (47.7) \\
\hline & Unknown & 124 & $(11.1)$ & 262 & $(15.8)$ & 128 & $(25.4)$ & 514 & (15.7) \\
\hline \multirow{2}{*}{$\begin{array}{l}\text { EAP } \\
\text { fulfilled } \\
\end{array}$} & Yes (or $\mathrm{n} / \mathrm{a}$ ) & 1,076 & $(96.2)$ & 1,601 & $(96.3)$ & 484 & $(96.0)$ & 3,161 & $(96.3)$ \\
\hline & No & 42 & $(3.8)$ & 61 & (3.7) & 20 & $(4.0)$ & 133 & (3.7) \\
\hline
\end{tabular}

a This is solely a reflection of a student's course-load at the institution. In other words, it fails to account for transient students and others who were concurrently enrolled at two or more institutions. In this study's urban locale, simultaneous cross-institutional enrollment is not uncommon, especially in light of Florida's statewide common course-numbering system.

${ }^{\mathrm{b}}$ Financial-aid (need-based) eligibility was measured by whether student was offered a Pell grant.

At the $\alpha=.05$ level, no significant differences between the three seat-time groups were observed for four of these covariates: financial aid $\left(\chi^{2}=2.998, d f=2, p=.223\right)$; SLS (student-life/learning) course attempted prior to Fall-2001 semester $\left(\chi^{2}=4.055, d f=2\right.$, $p=.132)$; SLS course passed prior to Fall-2001 semester $\left(\chi^{2}=5.571, d f=4, p=.062\right)$; and EAP (English-for-Academic-Purposes) requirement was either not applicable to student or was fulfilled by student $\left(\chi^{2}=0.096, d f=2, p=.953\right)$. Additionally, the 
significant test-statistic value $\left(\chi^{2}=12.809, d f=2, p=.002\right)$ detected for the verifieddisability covariate was judged irrelevant and likely due to chance, in light of the miniscule aggregate proportion (1.5\%) of students who were known by the institution to have a verified disability.

\section{Class-Section Characteristics Disaggregated by Primary IV}

Table 7 displays the distribution of seat-time levels for the 120 class sections (i.e., the Level-2 grouping) among which the population of Fall-2001 MAT0024 cohort students $(N=3,284)$ were subdivided. 
Table 7

Characteristics of Fall-2001 MAT0024 Class Sections

\begin{tabular}{|c|c|c|c|c|c|}
\hline & \multicolumn{3}{|c|}{ Seat Time } & \multirow{2}{*}{ Total } \\
\hline & & Short & Medium & Long & \\
\hline \multirow{2}{*}{\multicolumn{2}{|c|}{$\begin{array}{l}\text { \# of Class Sections } \\
\text { (\% of Class Sections) }\end{array}$}} & 40 & 60 & 20 & 120 \\
\hline & & $(33.3 \%)$ & $(50.0 \%)$ & $(16.7 \%)$ & $(100 \%)$ \\
\hline \multicolumn{2}{|c|}{ Mean Enrollment Per Section } & 28.0 & 27.7 & 25.2 & 27.4 \\
\hline \multicolumn{2}{|c|}{ Median Enrollment Per Section } & 29.0 & 29.0 & 27.5 & 29.0 \\
\hline \multicolumn{2}{|c|}{ Min-to-Max Enrollment Range } & $16-33$ & $13-34$ & $10-32$ & $10-34$ \\
\hline \multicolumn{2}{|c|}{ Enrollment $S D$ Per Section } & 3.2 & 4.2 & 6.7 & 4.5 \\
\hline \multirow{3}{*}{$\begin{array}{l}\text { Meeting } \\
\text { Time }\end{array}$} & Weekday & 40 & 43 & 1 & 84 \\
\hline & Evening & 0 & 17 & 14 & 31 \\
\hline & Weekend & 0 & 0 & 5 & 5 \\
\hline \multirow{3}{*}{$\begin{array}{l}\text { Session } \\
\text { Length }\end{array}$} & 16-week (full-term) & 39 & 43 & 17 & 99 \\
\hline & 11-week (mini-term) & 1 & 17 & 2 & 20 \\
\hline & 8-week (mini-term) & 0 & 0 & 1 & 1 \\
\hline \multicolumn{2}{|c|}{ Total\# of Class Mode } & 46 & 30 & 15 & $\overline{46}$ \\
\hline Meetings & Min-to-Max Range & $35-75$ & $22-31$ & $12-16$ & $12-75$ \\
\hline \multirow{5}{*}{$\begin{array}{l}\text { Campus } \\
\text { Locations }\end{array}$} & Central & 15 & 24 & 7 & 46 \\
\hline & Downtown & 0 & 2 & 4 & 6 \\
\hline & North & 7 & 16 & 6 & 29 \\
\hline & South+ Satellite Center ${ }^{\mathrm{a}}$ & 18 & 18 & 2 & 38 \\
\hline & Off-Campus ${ }^{\mathrm{b}}$ & 0 & 0 & 1 & 1 \\
\hline \multirow{5}{*}{$\begin{array}{l}\text { Enrollment pe } \\
\text { Section }\end{array}$} & 10-14 students & $\overline{0}$ & 2 & 1 & 3 \\
\hline & 15-19 students & 1 & 2 & 4 & 7 \\
\hline & $20-24$ students & 3 & 4 & 1 & 8 \\
\hline & $25-29$ students & 23 & 29 & 7 & 59 \\
\hline & 30-34 students & 13 & 23 & 7 & 43 \\
\hline \multicolumn{2}{|c|}{ Total \# of Students (\%) } & $\begin{array}{r}1,118 \\
(34.0 \%)\end{array}$ & $\begin{array}{r}1,662 \\
(50.6 \%)\end{array}$ & $\begin{array}{r}504 \\
(15.3 \%)\end{array}$ & $\begin{array}{r}3,284 \\
(100 \%)\end{array}$ \\
\hline
\end{tabular}

a South Campus's 38 class sections included 2 sections (1 short, 1 medium) taught at its nearby satellite center, which had opened the previous year.

boff-campus class was provided at an inner-city local high school during evening hours to 10 enrollees.

Each course section's total number of meetings was computed by the researcher, based upon the day(s) of the week the class met, the college's academic calendar, and (inclusive of) the final-exam-week schedule. So, the total number of meetings represents the pre-scheduled number of class meeting for each particular course. Of course, a particular course's actual number of meetings may be slightly less or slightly more; for 
example, less if the instructor was ill on a given day and no substitute instructor could be arranged, or more in the event an extra final-exam review session occurred.

As Table 7 shows, there was a wide range of total number of class meetings within each seat-time level. However, those ranges are somewhat misleading. For example, among the 40 short seat-time classes, 37 (92.5\%) of them had a total of 46 class meetings (i.e., MWF 16-week classes). The remaining three classes had 35, 60, and 75 total meetings, as elaborated upon in the next paragraph. Similarly, although the 60 medium seat-time classes ranged from 22 to 31 total class meetings, these values were essentially distributed in a bimodal manner: 43 sections met between 29 and 31 times, while the remaining 17 met either 22 or 24 times.

This clarification is important because it draws attention to the two (sometimes conflicting) factors that prompted the researcher to re-evaluate his initial operational definitions of the three levels of seat time: a class's seat-time duration (measured in minutes per meeting) versus the frequency/spacing of its meetings (measured in number of meeting days per week). A closer look at the classes placed at the short seat-time level illuminated this difference. The 40 short seat-time classes all met at least three days per week and had mean seat-time durations ranging from 50 to 90 minutes per meeting. Thirty-four of these 40 classes met three days per week (MWF) for 50 minutes per meeting in a full-length (16 week) semester, resulting in a total of 46 face-to-face class meetings; 32 of these 34 were traditional-lecture sections, while the remaining two were computer-enhanced with self-paced instructional software supplemented periodically by instructor-delivered mini-lectures. The remaining six of these 40 short seat-time classes were pilot-program offerings, which were created to evaluate whether additional 
classroom contact time improved student success rates. While all six of these classes were consistent in not charging the students additional tuition nor fees, their respective seat-time-apportionment and instructional-delivery formats varied as follows:

- Three of these classes - in contrast to the typical three hours per week of instructorclassroom contact—were comprised of five classroom hours per week over a fulllength semester span delivered in a traditional-lecture format. The seat-time apportionments for these three classes varied. One section met 5 days per week for 50 minutes per meeting resulting in a total of 75 class meetings; another met 4 days per week for an average of 62.5 minutes per meeting ( 75 minutes on TR, 50 minutes on MW); and one met 3 days per week (MWF) for 90 minutes per meeting resulting in a total of 46 class meetings throughout the full-length semester.

- Two of these classes had two 75-minute meetings per week (MW) with the instructor but were supplemented with a 60 -minute mandatory supplemental-instruction (recitation) session every Friday, resulting in a total of 46 class meetings throughout the full-length semester with an average seat time of 70 minutes per meeting;

- One of these classes was a computer-enhanced section comprised of three 80-minute face-to-face meetings per week (MWF) in a computerized classroom, resulting in 35 class meetings over an 11-week mini-session semester.

While it is true that that several of these six pilot-program classes had average permeeting seat times in excess of the 75-min duration common to the vast majority of the medium seat-time sections, the decision to ultimately classify all six at the short seat-time level, instead of the medium level, was based upon the fact that all of them met three or more times per week. Said differently, the spacing effect phenomenon, as discussed in 
Chapter 2's literature review, contrasts learning sessions that are shorter and more frequent/separated against those that are longer and more infrequent/massed. These six pilot-program classes fall into the former category, for each was comprised of more frequent/separated learning sessions. Hence, it is reasonable to conjecture that the higher frequency of class meetings compensated for the lengthier time duration of each meeting.

Lastly, there was one other explored aspect of class-section (Level-2) covariate characteristics that warranted some mention: instructor demography disaggregated by seat-time level. There were 66 instructors who taught the 120 Fall-2001 MAT0024 Elementary Algebra class sections. Among these 66 instructors, 55 taught their MAT0024 section(s) exclusively at one seat-time level; of which, 15 were short, 28 medium, and 12 long. The remaining 11 instructors taught Fall-2002 MAT0024 sections at more than one seat-time level; specifically, seven taught short- and medium-length sections, and the remaining four taught medium- and long-length sections.

With regard to tenure status, none of the 20 long seat-time sections were taught by instructors who had been tenured by the beginning of the Fall-2001 semester; that is, 15 sections were taught by part-time instructors and the remaining five by non-tenured fulltime faculty. In contrast, $52.5 \%$ ( 21 of 40 ) of the short seat-time sections were taught by tenured faculty, with the remaining $47.5 \%$ taught by part-time instructors. Of the 60 medium seat-time sections, $25 \%$ were taught by tenured full-time faculty, $10 \%$ by nontenured full-time faculty, and the remaining $65 \%$ by part-time instructors.

When disaggregated by student, there were 1,281 (39.0\%) enrollees in MAT0024 class sections taught by part-time faculty; hence, 2,003 (61.0\%) in sections taught by fulltime faculty. Among the 1,118 students who enrolled in short seat-time sections, 52.9\% 
( $n=591)$ were taught by full-time faculty. In this regard, students in medium and long seat-time sections had a course experience dissimilar from those in short seat-time sections; $32.8 \%(n=545)$ of medium and $28.8 \%(n=145)$ of long seat-time students were taught by full-time faculty.

\section{Analyses for Research Question 1}

The first of this study's three research questions was: In community-college developmental-level algebra courses, is there a difference in student success for students enrolled in classes that meet one day per week in longer-duration class meetings versus for those enrolled in medium-duration (twice weekly) class meetings versus for those enrolled in shorter-duration (thrice weekly) class meetings?

\section{Preliminary Cross-Tabulation Analyses}

The analysis of this question began with a simple cross-tabulation comparison of Fall-2001 MAT0024 course success and class-section seat-time level, which is displayed in Table 8. A chi-square test for homogeneity of proportions revealed no significant between-group difference $\left(\chi^{2}=2.004, d f=2, p=.367\right)$.

Table 8

Cross-Tabulation of Seat-Time Level Versus Course Success

\begin{tabular}{|c|c|c|c|c|c|c|c|c|}
\hline \multirow{3}{*}{$\begin{array}{l}\text { Passed } \\
\text { MAT0024 } \\
\text { Course? }\end{array}$} & \multicolumn{6}{|c|}{ Seat-Time Duration } & \multirow{2}{*}{\multicolumn{2}{|c|}{$\begin{array}{c}\text { Total } \\
(N=3,284)\end{array}$}} \\
\hline & \multicolumn{2}{|c|}{$\begin{array}{c}\text { Short } \\
(n=1,118)\end{array}$} & \multicolumn{2}{|c|}{$\begin{array}{c}\text { Medium } \\
(n=1,662) \\
\end{array}$} & \multicolumn{2}{|c|}{$\begin{array}{c}\text { Long } \\
(n=504)\end{array}$} & & \\
\hline & $n$ & $(\%)$ & $n$ & $(\%)$ & $n$ & $(\%)$ & $n$ & $(\%)$ \\
\hline Yes & 490 & (43.8) & 698 & $(42.0)$ & 228 & $(45.2)$ & 1,416 & $(43.1)$ \\
\hline $\mathrm{No}^{\mathrm{a}}$ & 628 & $(56.2)$ & 964 & $(58.0)$ & 276 & $(54.8)$ & 1,868 & $(56.9)$ \\
\hline
\end{tabular}

a Non-passing grades consisted of: 128 D's, 1123 F's, 594 W's (Withdrawals - either student or instructor initiated), 6 NG's (instructor assigned "No-Grade" in lieu of D or F), and 17 NR (missing - grade "Not Reported" by instructor). 
Then, a cursory examination of inter-campus student success rates was then conducted, in order to assess whether a three-level hierarchical logistic regression analysis should be pursued or whether a two-level analysis would be sufficient. Table 9 below shows Fall-2001 MAT0024 course success rates disaggregated by the course's location (i.e., campus, satellite center, remote non-college facility). No significant between-location differences were observed $\left(\chi^{2}=5.579, d f=4, p=.233\right)$.

Table 9

Cross-Tabulation of Class Location Versus Course Success

\begin{tabular}{|c|c|c|c|c|c|c|c|c|c|c|}
\hline \multirow{3}{*}{$\begin{array}{l}\text { Passed } \\
\text { MAT0024 } \\
\text { Course? }\end{array}$} & \multicolumn{10}{|c|}{ Class Campus/Center Location } \\
\hline & \multicolumn{2}{|c|}{$\begin{array}{c}\text { Central } \\
(n=1,261)\end{array}$} & \multicolumn{2}{|c|}{$\begin{array}{c}\text { North } \\
(n=821)\end{array}$} & \multicolumn{2}{|c|}{$\begin{array}{c}\text { South }+ \\
\text { Satellite Ctr } \\
(n=1,055)\end{array}$} & \multicolumn{2}{|c|}{$\begin{array}{c}\text { Downtown } \\
(n=137)\end{array}$} & \multicolumn{2}{|c|}{$\begin{array}{c}\text { Off-Campus } \\
\quad(n=10)\end{array}$} \\
\hline & $f$ & $(\%)$ & $f$ & $(\%)$ & $f$ & $(\%)$ & $f$ & $(\%)$ & $f$ & $(\%)$ \\
\hline Yes & 522 & (41.4) & 355 & $(43.2)$ & 474 & (44.9) & 63 & (46.0) & 2 & (20.0) \\
\hline No & 739 & (58.6) & 466 & $(56.8)$ & 581 & $(55.1)$ & 74 & $(54.0)$ & 8 & $(80.0)$ \\
\hline
\end{tabular}

\section{Construction and Testing of Multilevel Logistic-Regression Models}

As previously discussed, there were two main characteristics of the multivariable data collected to investigate this first research question that determined the choice of analysis technique.

First, the Fall-2001 cohort data were multilevel in nature; that is to say, students were nested within class sections, and class sections were, in turn, nested within campuses. Mullens, Murnane, and Willett (1996) contend:

Students within a classroom are likely to be more homogeneous than are students in different classrooms. The analysis of such data with single-level techniques... violates the assumption of independent observations implicit in these methods and may result in incorrect standard error estimation and flawed hypothesis tests. (p. 143) 
Various other scholars support this view (e.g., p. 4 of Raudenbush \& Bryk, 2002; pp. 4-5 of Hox, 2010).

Consequently, the variables that comprised this study's data set possessed an inherent hierarchical structure. Due, however, to the aforementioned analysis of betweencampus success rates and, more importantly, the fact that it is not at all uncommon for a student in a given semester to enroll in courses at multiple campuses, the researcher decided to not pursue campus-level (Level-3) variables. Instead, a two-level logistic regression analysis was conducted. That is to say, the broad method of analysis determined to be most applicable to this study's first research question is an extension of the concept of the generalized linear model (GLM). Partly due to proprietary differences in software terminology, the method is known by a wide variety of different (but similar) names, including: generalized linear mixed modeling or GLMM (Heck, Thomas, \& Tabata, 2012); multilevel generalized linear modeling (Hox, 2010); multilevel linear modeling or MLM (Tabachnick \& Fidell, 2007); and hierarchical linear modeling or HLM (Raudenbush \& Bryk, 2002). Despite disparities in name, they are unified in purpose and approach: to provide a means to analyze and describe relationships among variables that have been measured at varying levels in a hierarchical data structure

The second aspect of the data set that guided the researcher's analysis-technique selection was: the dependent variable of interest (student success) is a binary outcome (pass or fail). This lead to the decision to use multilevel logistic regression, a subcategory of GLMM/MLM. 
Step 1a: Single-Level (Intercept-Only) Model. To assess whether a multilevel model was necessary, the following single-level generalized linear model was created with SPSS's GENLIN routine:

$$
E\left(Y_{i}\right)=\pi
$$

which denotes that the expected value of an individual cohort student $i$ having been successful in their Fall-2001 MAT0024 course attempt equals $\pi$, where $\pi$ is the probability that $Y_{i}=1$ (i.e., student was successful, having earned a passing course grade, defined by the state as a "C" or higher). Hence, the complementary probability $1-\pi$ is the probability that $Y_{i}=0$ (i.e., student was unsuccessful, having earned a non-passing grade or a withdrawal grade, etc.).

Since the outcome variable was dichotomous, the intercept-only model utilizes the binomial probability distribution and the logit link (transformation) function to relate the transformed predicted $Y_{i}$ values to an estimated intercept parameter as follows:

$$
\eta_{i}=\operatorname{logit}(\pi)=\ln \left(\frac{\pi}{1-\pi}\right)=\beta_{0}
$$

where $l n$ denotes the natural (base $e$ ) logarithm. By mathematical definition, the ratio formed by dividing the probability of success $\pi$ by the probability of failure $1-\pi$ represents what is termed the odds in favor of a student being successful. Consequently, the intercept $\beta_{0}$ represents the log odds.

The purpose of this intercept-only model was to establish a preliminary baseline predictive-accuracy rate, to which subsequent models — of both the single-level (GLM) and the multilevel (GLMM) type-could be compared. 
SPSS's GENLIN program was used to create and test the GLM models. After changing GENLIN's covariance-matrix setting from the default (model-based estimator) option to the robust estimator setting and switching the log-likelihood function setting from full to kernel, the following output (displayed in Table 10) was obtained:

Table 10

Intercept-Only Single-Level (GLM) Model

95\% Wald C.I.

Std. 95\% Wald C.I. Hypothesis Test $\quad$ for $\operatorname{Exp}(\beta)$

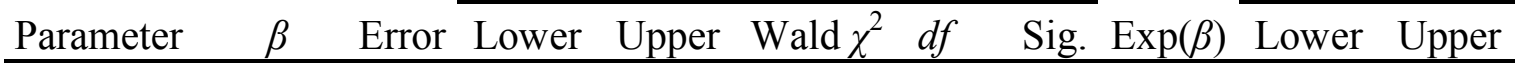

\begin{tabular}{llllllllllll}
\hline (Intercept) & -0.277 & .035 & -0.346 & -0.208 & 61.816 & 1 & $<.001$ & 0.758 & 0.707 & 0.812
\end{tabular}
(Scale) $\quad 1^{\text {a }}$

Dependent Variable: Passed Course Model: (Intercept)

a. Fixed at the displayed value.

Categorical Variable Information

\begin{tabular}{|c|c|c|c|c|}
\hline & & & $N$ & Percent \\
\hline \multirow{3}{*}{$\begin{array}{l}\text { Dependent } \\
\text { Variable }\end{array}$} & \multirow{3}{*}{ Passed Course } & No & 1,868 & $56.9 \%$ \\
\hline & & Yes & 1,416 & $43.1 \%$ \\
\hline & & Total & 3,284 & $100.0 \%$ \\
\hline
\end{tabular}

The logistic function's $y$-intercept value of -0.277 represents the natural logarithm of the odds that a randomly-selected student passed the Fall-2001 MAT0024 course with a grade of at least a "C" (i.e., $Y_{i}=1$ ). Because a $\log$ odds scale is difficult to interpret, the natural logarithm's inverse function was computed, which is represented in Table 10 as $\operatorname{Exp}(\beta)$. That is, $\operatorname{Exp}(\beta)=e^{\beta}=e^{-0.277} \approx 0.758$, which represents the mathematical odds in favor of a student passing the course (i.e., the ratio of the probability of passing the course to the probability of not passing). The reciprocal value $1 / 0.758 \approx 1.319$, therefore, represents the odds against a student passing the course. This indicates that, in the population of MAT0024 students at this multi-campus institution, it is estimated that students are approximately 1.3 times more likely to not pass than they are to pass. The 
categorical variable information shown in Table 8, as well as Table 10, provides confirmation of this interpretation; that is, dividing the 1,868 (56.9\%) MAT0024 successful students by the $1,416(43.1 \%)$ unsuccessful students yields a ratio of 1.319 .

Step 1b: Single-Level Model with Seat-Time Predictor Only. Seat-time level (ST) was then added to the model, with it coded as $0=$ short, $1=$ medium, and $2=$ long. To be consistent with research hypothesis $\mathrm{H}_{1}$, the short seat-time level was used as the reference category. Seat time was found to not be a significant predictor, as the Wald chisquare values in Table 11 below indicate.

Table 11

Single-Level (GLM) Model with Seat-Time as Sole IV

95\% Wald C.I.

Std. 95\% Wald C.I. Hypothesis Test for $\operatorname{Exp}(\beta)$

\begin{tabular}{lrrrrrrrrrrr} 
Parameter & $\beta$ & Error & Lower & Upper & Wald $\chi^{2}$ & $d f$ & Sig. & $\operatorname{Exp}(\beta)$ & Lower & Upper \\
\hline (Intercept) & -0.277 & .035 & -0.346 & -0.208 & 61.816 & 1 & $<.001$ & 0.758 & 0.707 & 0.812 \\
{$[\mathrm{ST}=2]$} & 0.057 & .107 & -0.154 & 0.269 & 0.280 & 1 & .597 & 1.059 & 0.857 & 1.308 \\
\hline$[\mathrm{ST}=1]$ & -0.075 & .078 & -0.228 & 0.078 & 0.915 & 1 & .339 & 0.928 & 0.796 & 1.082 \\
\hline$[\mathrm{ST}=0]$ & $0^{\mathrm{a}}$ & & & & & & & 1 & & \\
\hline \multicolumn{1}{c}{$(\mathrm{Scale})$} & $1^{\mathrm{b}}$ & & & & & & & & & \\
\hline
\end{tabular}

Dependent Variable: Passed Course Model: (Intercept), Seat Time

a. Set to zero because the parameter is redundant.

b. Fixed at the displayed value.

To test the difference between this fitted model and the intercept-only model, the likelihood-ratio chi-square omnibus test was performed. This test statistic reveals the difference in $-2 \times \log$ likelihoods between the two models. The estimate obtained $\left(\chi^{2}=\right.$ 2.002, $d f=2, p=.367$ ) confirmed no significant improvement in predictive capacity between the models.

It should be noted that the model was then re-run with the trichotomous seat-time variable re-coded in two alternative binary ways: (a) short seat time $(1=\mathrm{Yes}, 0=\mathrm{No})$; and 
(b) not-long seat time ( $1=$ Yes, representing short or medium; $0=$ No, representing long).

Their respective likelihood-ratio chi-square values were $.348(p=.555)$ and $1.088(p=$ .297). Hence, neither resulted in a significant improvement compared to the interceptonly model.

Step 2a: Two-Level Unconditional (Null, Intercepts-Only) Model. Attention was then turned to the multilevel (GLMM) model, in an effort to first quantify the degree of variability in the Level-1 outcome variable (MAT0024 course success) across class/section (Level-2) units.

Each class section's institutionally-assigned, unique reference number served as the Level-2 grouping variable. Then, with the aid of the GENLIN-MIXED routine (and the robust-covariances setting) in SPSS version 20, the unconditional model was obtained. Table 12 summarizes that output and its predictive accuracy.

Table 12

Two-Level GLMM Null (Intercepts-Only) Model

Fixed Effects

\begin{tabular}{lccccccc}
\hline Model & & Std. & \multicolumn{3}{c}{ Hypothesis Test } & & \multicolumn{2}{c}{$95 \%$ C.I. for Exp(Coeff) } \\
\cline { 3 - 8 } Term & Coeff. & Error & $t$ & Sig. & $\operatorname{Exp}($ Coeff) & Lower & Upper \\
\hline Intercept & -0.290 & .054 & -5.357 & $<.001$ & 0.748 & 0.673 & 0.832 \\
\hline Probability Distribution: Binomial & & & & \\
Link Function: Logit \\
Target: Passed Course [Grouping Variable: Class Reference \#] \\
\hline Random Effect
\end{tabular}

\begin{tabular}{|c|c|c|c|c|c|c|}
\hline \multirow[b]{2}{*}{ Model Term } & \multirow[b]{2}{*}{ Estimate } & \multirow[b]{2}{*}{ Std. Error } & \multicolumn{2}{|c|}{ Hypothesis Test } & \multicolumn{2}{|c|}{$95 \% \mathrm{CI}$} \\
\hline & & & $\mathrm{z}$ & Sig. & Lower & Upper \\
\hline $\operatorname{Var}($ Intercept $)$ & 0.198 & .046 & 4.265 & $<.001$ & 0.125 & 0.314 \\
\hline \multicolumn{7}{|c|}{$\begin{array}{l}\text { Covariance Structure: Variance components } \\
\text { Subject Specification: Class Reference Number }\end{array}$} \\
\hline \multicolumn{7}{|c|}{ Overall Predictive Accuracy Rate $=62.4 \%$} \\
\hline & \multicolumn{3}{|c|}{ Predicted } & & & \\
\hline Observed & Success & Failu & & & & \\
\hline Success & $31.1 \%$ & 68.9 & & & & \\
\hline Failure & $13.9 \%$ & 86.1 & & & & \\
\hline
\end{tabular}


Because the fixed-effects intercept value of -0.290 is expressed in terms of the logit link's log-odds scale, it must first be converted to a probability, by computing the mathematical inverse function of the one-to-one logit-link function:

$$
\frac{1}{1+e^{-(-0.290)}} \approx 0.428
$$

This represents the estimated average class-level (Level-2) probability of a random student passing the MAT0024 course. It, therefore, follows that the estimated average class-level probability of a student not passing the MAT0024 course is $42.8 \%$, the complementary event's likelihood. It should be noted that these two probability values are slightly different than those of the single-level (i.e., student-level) GLM analysis previously discussed, which were $43.1 \%$ passing and $56.9 \%$ not passing.

The results of the $z$-test for the variance component $(z=4.265, p<.001)$ show that the intercept variance fluctuates significantly between the 120 class-section (Level-2) groupings. This supports the necessity of performing a multilevel-modeling analysis.

To quantify the percent of the variability in passing rates that was accounted for by between-class (Level-2) differences, the intraclass correlation (ICC) was computed. The ICC (denoted by $\rho$ ) represents the proportion of variance that lies between units (i.e., class sections) relative to the total variance. The within-unit (Level-1) variance of the standard logit distribution equals $\frac{\pi^{2}}{3} \approx 3.29$ (Heck et al., 2012; Hox, 2010). Hence, the ICC's value was computed as follows:

$$
\rho=\frac{\sigma_{\text {between }}^{2}}{\sigma_{\text {between }}^{2}+\sigma_{\text {within }}^{2}}=\frac{\sigma_{\text {between }}^{2}}{\sigma_{\text {between }}^{2}+\frac{\pi^{2}}{3}} \approx \frac{0.198}{0.198+3.29} \approx 0.0568
$$


This means that about $5.7 \%$ of the variability in MAT0024 course success is attributable to between-class (Level-2) variability.

It should be noted that the intercepts-only model was run in a second, alternative way of grouping the students: by making the Level-2 grouping variable the instructor dummy identification (ID) code, instead of the class-section reference number. Although this reduced the number of common (Level-2) subjects from 120 classes down to 66 instructors, the resulting null-model's intercept $(\beta=-0.281)$, variance-component test result $(z=3.623, p<.001)$, and its corresponding ICC $(\rho=.0562)$ were nearly the same as those of the class-section grouping. However, the predictive accuracy of this model was $65.2 \%$, a nearly $3 \%$ improvement over the $62.4 \%$ obtained with the class-section grouping.

Step 2b: Multi-Level Model (GLMM) with Seat-Time Predictor Only. Seattime level (ST) was then added to the GLMM model as a lone IV. As was the case with the single-level GLM model, seat time was found to not be a significant predictor, as Table 13 below demonstrates. In fact, the predictive accuracy of this fitted model was $62.2 \%$, lower than the $62.4 \%$ of the intercepts-only GLMM model.

Table 13

Two-Level (GLMM) Model with Seat-Time as Sole IV 95\% CI for

\begin{tabular}{|c|c|c|c|c|c|c|c|}
\hline \multirow[b]{2}{*}{ Parameter } & \multirow[b]{2}{*}{$\beta$} & \multirow{2}{*}{$\begin{array}{c}\text { Std. } \\
\text { Error }\end{array}$} & \multicolumn{2}{|c|}{ Hypothesis Test } & \multirow[b]{2}{*}{$\operatorname{Exp}(\beta)$} & \multicolumn{2}{|c|}{$\operatorname{Exp}(\beta)$} \\
\hline & & & $t$ & Sig. & & Lower & Upper \\
\hline (Intercept) & -0.249 & .077 & -3.225 & .001 & 0.780 & 0.670 & 0.907 \\
\hline$[\mathrm{ST}=2]$ & 0.037 & .173 & 0.212 & .832 & 1.037 & 0.739 & 1.456 \\
\hline$[\mathrm{ST}=1]$ & -0.094 & .111 & -0.841 & .400 & 0.911 & 0.732 & 1.133 \\
\hline
\end{tabular}

$[\mathrm{ST}=0] \quad 0^{\mathrm{a}}$

Probability distribution: Binomial Link function: Logit

a. This coefficient is set to zero because it is redundant. 
Step 2c: Demographic Covariates added to Multi-Level (GLMM) Model. Six demographic-type (Level-1) covariates were then added to the preceding step's model: sex $(0=$ male, $1=$ female $)$; student age; ethnicity $(0=$ Non-Hispanic, $1=$ Hispanic, $2=$ unreported $)$; race $(0=$ White, $1=$ Asian, $2=$ Black, $3=$ Native-American, $4=$ Pacific Islander, $5=$ unreported $)$; a dichotomous recoding of birthplace $(0=\mathrm{U} . \mathrm{S}$. born, $1=$ foreign born); and a consolidation of immigration status types $(0=\mathrm{U} . \mathrm{S}$. citizen, $1=$ Permanent Resident Alien, 2=F-1 Student Visa, 3=Other Documented Type,4=Undocumented). In light of the high rate of missing values for race and ethnicity, these two variables were recoded in such a way that the missing values were assigned a code, so that this fitted model would not rely upon a significantly smaller sample size than the previous one. Additionally, the one continuous demographic covariate, student age, was grand mean centered. This model, which yielded a $64.1 \%$ predictive accuracy rate with $n=3,274$ (i.e., 10 excluded cases due to missing values, four sex and six birthplace), is summarized in Table 14. 
Table 14

Two-Level Model with Seat-Time and Demographic Covariates

\begin{tabular}{|c|c|c|c|c|c|c|c|}
\hline \multirow[b]{2}{*}{ Parameter } & \multirow[b]{2}{*}{$\beta$} & \multirow{2}{*}{$\begin{array}{l}\text { Std. } \\
\text { Error }\end{array}$} & \multicolumn{2}{|c|}{ Hypothesis Test } & \multirow[b]{2}{*}{$\operatorname{Exp}(\beta)$} & \multicolumn{2}{|c|}{$\begin{array}{c}95 \% \mathrm{CI} \text { for } \\
\operatorname{Exp}(\beta)\end{array}$} \\
\hline & & & $t$ & Sig. & & Lower & Upper \\
\hline (Intercept) & -0.386 & .172 & -2.247 & $.025 *$ & .680 & 0.486 & 0.952 \\
\hline$[\mathrm{ST}=2] \mathrm{L}$ & -0.073 & .188 & -.387 & .699 & .930 & 0.644 & 1.344 \\
\hline$[\mathrm{ST}=1] \mathrm{M}$ & -0.130 & .118 & -1.104 & .270 & .878 & 0.697 & 1.106 \\
\hline$[\mathrm{ST}=0] \mathrm{S}^{\mathrm{a}}$ & $0^{\mathrm{a}}$ & & & & & & \\
\hline $\mathrm{Sex}=1$ & 0.283 & .074 & 3.850 & $<.001^{*}$ & 1.327 & 1.149 & 1.533 \\
\hline $\operatorname{Sex}=0^{a}$ & $0^{\mathrm{a}}$ & & & & & & \\
\hline Ethn=2 & 0.119 & .144 & 0.825 & .410 & 1.126 & 0.849 & 1.493 \\
\hline Ethn $=1$ & 0.048 & .237 & 0.204 & .839 & 1.049 & 0.660 & 1.670 \\
\hline Ethn $=0^{\text {a }}$ & $0^{\mathrm{a}}$ & & & & & & \\
\hline Race $=5$ & -0.158 & .214 & -0.739 & .460 & .854 & 0.561 & 1.299 \\
\hline Race $=3$ & -0.395 & .602 & -0.656 & .512 & .674 & 0.207 & 2.193 \\
\hline Race $=2$ & -0.550 & .100 & -5.499 & $<.001 *$ & .577 & 0.474 & 0.702 \\
\hline Race $=1$ & -0.175 & .254 & -0.688 & .492 & .840 & 0.510 & 1.382 \\
\hline Race $=0^{\mathrm{a}}$ & $0^{\mathrm{a}}$ & & & & & & \\
\hline Birthpl=1 & 0.036 & .135 & 0.267 & .790 & 1.037 & 0.795 & 1.352 \\
\hline Birthpl $=0^{a}$ & $0^{\mathrm{a}}$ & & & & & & \\
\hline Immig=4 & 1.159 & 1.150 & 1.008 & .314 & 3.187 & 0.334 & 30.370 \\
\hline Immig=3 & 1.057 & .265 & 3.985 & $<.001 *$ & 2.877 & 1.710 & 4.838 \\
\hline Immig=2 & 1.758 & .305 & 5.762 & $<.001^{*}$ & 5.799 & 3.188 & 10.547 \\
\hline Immig=1 & 0.296 & .145 & 2.042 & $.041 *$ & 1.344 & 1.012 & 1.785 \\
\hline Immig $=0^{\mathrm{a}}$ & $0^{\mathrm{a}}$ & & & & & & \\
\hline AgeGMctr & 0.017 & .005 & 3.259 & $.001 *$ & 1.017 & 1.007 & 1.028 \\
\hline
\end{tabular}

Among the 21 fixed effects shown (that is, in addition to the intercept), six were shown to be significantly related to success in the MAT0024 course: sex; age; whether one's race was Black/African-American; and whether one's immigration status at the time of the course was Permanent Resident Alien, an F-1 Student Visa, or another documented type. Once again, seat-time level failed to be a significant predictor of course success. 
To sufficiently illustrate how to interpret these results, consider three of these significant covariates: F-1 Student Visa (immig=2), Black (race=2), and age (grand-mean centered). First, the model's estimated coefficient value for F-1 Student Visa $(\beta=1.758)$ means that being a student who possesses such a visa increases the log odds of being successful in the MAT0024 course by 1.758 units, when all the other effects are held constant. Of course, as previously explained, the logit link's log-odds scale does not immediately lend itself to user-friendly interpretation. Hence, it is better to make use of the exponentiated value of the coefficient (i.e., $e^{1.758} \approx 5.799$ ), as shown in the $\operatorname{Exp}(\beta)$ column within the table, along with its accompanying confidence interval. This 5.799 value indicates that the estimated odds of being successful in MAT0024 are almost six times higher for F-1 Student Visa students, when all the other covariates are held constant.

Second, and similarly, the Black/African-American coefficient value $(\beta=-0.550)$ indicates that being Black/African-American decreases the log odds of being successful in the MAT0024 course by 0.550 units. The exponentiated value of 0.577 represents the odds ratio; that is, the odds that a Black student is successful in MAT0024—after adjusting for all other covariates - are multiplied by 0.577 , when compared to non-Black students. In other words, the odds of a Black student being successful in MAT0024 are reduced by about $42.3 \%$. However, it is important to reiterate that, unlike immigration status, race and ethnicity were variables with a high proportion of unreported values and (due to the lack of verification processes) low internal consistency.

Thirdly, while the previous two illustrations demonstrate how to interpret the model's nominal-level, discrete covariates, those explanations require some modification 
in order to properly interpret the one ratio-level, continuous covariate: student age. Because age was grand-mean centered, the mean student age is represented by an age value of 0 . This, then, enables the exponentiated coefficient value of 1.017 to be interpreted as follows: the odds of being successful in MAT0024 are increased 1.017 times (i.e., 1.7\%) for each one-year unit increase in age, with all other covariates held constant. So, for example, a five-year increase in age would equate to an estimated $8.8 \%$ increase (i.e., $1.017^{5} \approx 1.088$ ) in the odds of being successful in MAT0024.

\section{Step 2d: Academic-Background Covariates added to Multi-Level (GLMM)}

Model. The next model constructed consisted of the seat-time IV accompanied by the following academic-background covariates: whether a student-life/learning-skills (SLS) course was attempted prior to the Fall-2001 MAT0024 course ( $0=$ No, $1=$ Yes $)$; whether a passing grade in an SLS course was earned prior to the Fall-2001 term $(0=\mathrm{No}, 1=\mathrm{Yes})$; total number of semester hours student attempted and total number earned (i.e., two variables) within the college and at other postsecondary institutions prior to the Fall-2001 semester, with both variables grand-mean centered; financial-aid eligibility $(0=$ Pell not offered, $1=$ Pell grant offered); institutionally verified disability ( $0=$ No, $1=$ Yes $)$; student's Fall-2001 course load at institution $(0=$ Part-Time, $1=$ Full-Time $)$; whether student completed or was exempt from non-native speaker EAP coursework prior to Fall-2001 $(0=$ No, $1=$ Yes $)$; and tuition/fee rate paid in Fall-2001 ( $0=$ In-state/resident, $1=$ Out-ofstate/nonresident).

There were no excluded cases in this model (i.e., $n=3,284)$. Its predictive accuracy (64.1\%) was identical to the preceding demographic-background model. The fixed-effects output is presented in Table 15. 
Table 15

Two-Level Model with Seat-Time and Academic Covariates

\begin{tabular}{|c|c|c|c|c|c|c|c|}
\hline \multirow[b]{2}{*}{ Parameter } & \multirow[b]{2}{*}{$\beta$} & \multirow{2}{*}{$\begin{array}{l}\text { Std. } \\
\text { Error }\end{array}$} & \multicolumn{2}{|c|}{ Hypothesis Test } & \multirow[b]{2}{*}{$\operatorname{Exp}(\beta)$} & \multicolumn{2}{|c|}{$\begin{array}{c}95 \% \mathrm{CI} \text { for } \\
\operatorname{Exp}(\beta)\end{array}$} \\
\hline & & & $t$ & Sig. & & Lower & Upper \\
\hline (Intercept) & 0.240 & .207 & 1.158 & .247 & 1.271 & 0.847 & 1.907 \\
\hline$[\mathrm{ST}=2] \mathrm{L}$ & 0.106 & .170 & 0.625 & .532 & 1.112 & 0.797 & 1.552 \\
\hline$[\mathrm{ST}=1] \mathrm{M}$ & -0.067 & .116 & -0.575 & .566 & 0.936 & 0.745 & 1.174 \\
\hline$[\mathrm{ST}=0] \mathrm{S}$ & $0^{\mathrm{a}}$ & & & & & & \\
\hline SLS-Att $=1$ & -0.413 & .214 & -1.927 & .054 & 0.662 & 0.435 & 1.007 \\
\hline SLS-Att $=0$ & $0^{\mathrm{a}}$ & & & & & & \\
\hline SLS-Pas $=1$ & -0.059 & .220 & -0.270 & .787 & 0.942 & 0.612 & 1.451 \\
\hline SLS-Pas $=0$ & $0^{\mathrm{a}}$ & & & & & & \\
\hline Hrs-Attmp & -0.025 & .005 & -5.108 & $<.001 *$ & 0.975 & 0.966 & 0.985 \\
\hline Hrs-Passed & 0.032 & .006 & 5.005 & $<.001 *$ & 1.033 & 1.020 & 1.046 \\
\hline Pell=1 & -0.224 & .083 & -2.680 & $.007 *$ & 0.800 & 0.679 & 0.942 \\
\hline Pell $=0$ & $0^{\mathrm{a}}$ & & & & & & \\
\hline Disabled $=1$ & -0.323 & .360 & -0.898 & .369 & 0.724 & 0.358 & 1.465 \\
\hline Disabled $=0$ & $0^{\mathrm{a}}$ & & & & & & \\
\hline $\mathrm{FT}-\mathrm{PT}=1$ & 0.148 & .089 & 1.667 & .096 & 1.159 & 0.974 & 1.379 \\
\hline FT-PT $=0$ & $0^{\mathrm{a}}$ & & & & & & \\
\hline $\mathrm{EAP}=1$ & -0.469 & .186 & -2.529 & $.011 *$ & 0.626 & 0.435 & 0.900 \\
\hline $\mathrm{EAP}=0$ & $0^{\mathrm{a}}$ & & & & & & \\
\hline Tuition=1 & 0.448 & .130 & 3.450 & $.001 *$ & 1.565 & 1.213 & 2.018 \\
\hline Tuition $=0$ & $0^{\mathrm{a}}$ & & & & & & \\
\hline
\end{tabular}

In addition to the five asterisk-marked covariates with significant $p$-values, two others predictors (specifically, tuition status and SLS attempted with $p<.10$ for both) merited at least preliminary inclusion in subsequent fitted models.

\section{Step 2e: Class-Section (Level-2) Covariates added to Multi-Level (GLMM)}

Model. The next model was comprised of the seat-time IV supplemented by the following class-section covariates: campus location $(1=$ Central, $2=$ North, $3=$ South + 
Satellite Center, 4=Downtown, 5=Off-Site); instructor sex $(0=$ male, $1=$ female); instructor age (grand-mean centered) at time of course's Fall-2001 session-start date; tenure status in Fall-2001 semester $(0=$ tenured, $1=$ untenured $)$; full-time/part-time status $(0=$ full-time, $1=$ adjunct/part-time instructor). With a classification predictive accuracy rate of $62.2 \%$ based on the full sample $(N=3,284)$, this model's fixed-effects output is shown in Table 16.

Table 16

Two-Level Model with Seat-Time and Level-2 Covariates

\begin{tabular}{|c|c|c|c|c|c|c|c|}
\hline \multirow[b]{2}{*}{ Parameter } & \multirow[b]{2}{*}{$\beta$} & \multirow{2}{*}{$\begin{array}{l}\text { Std. } \\
\text { Error }\end{array}$} & \multicolumn{2}{|c|}{ Hypothesis Test } & \multirow[b]{2}{*}{$\operatorname{Exp}(\beta)$} & \multicolumn{2}{|c|}{$\begin{array}{c}95 \% \text { CI for } \\
\operatorname{Exp}(\beta)\end{array}$} \\
\hline & & & $t$ & Sig. & & Lower & Upper \\
\hline (Intercept) & -0.420 & .119 & -3.532 & $<.001$ & 0.657 & 0.520 & 0.830 \\
\hline$[\mathrm{ST}=2] \mathrm{L}$ & 0.266 & .238 & 1.119 & .263 & 1.305 & 0.819 & 2.080 \\
\hline$[\mathrm{ST}=1] \mathrm{M}$ & 0.039 & .138 & 0.283 & .777 & 1.040 & 0.794 & 1.361 \\
\hline$[\mathrm{ST}=0] \mathrm{S}$ & $0^{\mathrm{a}}$ & & & & & & \\
\hline Location $=5$ & -1.530 & .227 & -6.742 & $<.001 *$ & 0.217 & 0.139 & 0.338 \\
\hline Location $=4$ & -0.127 & .363 & -0.349 & .727 & 0.881 & 0.432 & 1.795 \\
\hline Location $=3$ & 0.160 & .123 & 1.296 & .195 & 1.173 & 0.921 & 1.494 \\
\hline Location $=2$ & -0.028 & .147 & -0.190 & .849 & 0.972 & 0.728 & 1.298 \\
\hline Location $=1$ & $0^{\mathrm{a}}$ & & & & & & \\
\hline PrfGndr $=1$ & 0.166 & .119 & 1.400 & .162 & 1.180 & 0.936 & 1.489 \\
\hline PrfGndr $=0$ & $0^{\mathrm{a}}$ & & & & & & \\
\hline PrfAgeGM & -0.010 & .004 & -2.422 & $.015^{*}$ & 0.990 & 0.981 & 0.998 \\
\hline Untenrd $=1$ & -0.161 & .221 & -0.730 & .466 & 0.851 & 0.552 & 1.313 \\
\hline Untenrd $=0$ & $0^{\mathrm{a}}$ & & & & & & \\
\hline Adjnct $=1$ & 0.127 & .186 & 0.683 & .495 & 1.136 & 0.788 & 1.636 \\
\hline Adjnct $=0$ & $0^{\mathrm{a}}$ & & & & & & \\
\hline
\end{tabular}

The only covariate of predictive significance was the instructor age $(p=.015)$. The other covariate with a "significant" $p$-value was the off-site class location $(p<.001)$. However, this was spurious, in that (a) only 10 students $(0.3 \%$ of the sample) were enrolled in the 
one off-site location (among the sample's 120 class sections), and (b) that section's atypically low (20\%) student-success rate was an overly influential outlier. Hence, it was disregarded.

Step 3: Final Multi-Level (GLMM) Fitted Model. To ultimately obtain a parsimonious model, a series of successive SPSS runs were performed. The modelbuilding strategy employed was to begin with any covariates in the preceding runs that displayed $p$-values not exceeding .10, and gradually pare the model until only significant $(p<.05)$ covariates remained. That model, as detailed in Table 17, yielded a $65.9 \%$ predictive accuracy rate and was based upon a sample size of 3,280 cohort students (i.e., four subjects were excluded due to a missing covariate value). 
Table 17

Final Two-Level GLMM Model

\begin{tabular}{|c|c|c|c|c|c|c|c|}
\hline \multirow[b]{3}{*}{ Parameter } & \multirow[b]{3}{*}{$\beta$} & \multirow{2}{*}{\multicolumn{3}{|c|}{$\begin{array}{l}\text { Hypothesis } \\
\text { Test }\end{array}$}} & \multirow[b]{3}{*}{$\operatorname{Exp}(\beta)$} & \multirow{2}{*}{\multicolumn{2}{|c|}{$\begin{array}{c}95 \% \mathrm{CI} \text { for } \\
\operatorname{Exp}(\beta)\end{array}$}} \\
\hline & & & & & & & \\
\hline & & $\begin{array}{l}\text { Std. } \\
\text { Error }\end{array}$ & $t$ & Sig. & & Lower & Upper \\
\hline (Intercept) & -0.577 & .111 & -5.215 & $<.001$ & 0.562 & 0.452 & 0.698 \\
\hline Stud Age GM & 0.030 & .006 & 5.009 & $<.001$ & 1.030 & 1.018 & 1.042 \\
\hline Student Sex $=1$ & 0.320 & .077 & 4.176 & $<.001$ & 1.377 & 1.185 & 1.601 \\
\hline Student Sex $=0$ & $0^{\mathrm{a}}$ & & & & & & \\
\hline Black Race $=1$ & -0.443 & .085 & -5.200 & $<.001$ & 0.642 & 0.544 & 0.759 \\
\hline Black Race $=0$ & $0^{\mathrm{a}}$ & & & & & & \\
\hline SLS Attempted=1 & -0.335 & .092 & -3.652 & $<.001$ & 0.715 & 0.598 & 0.856 \\
\hline SLS Attempted $=0$ & $0^{\mathrm{a}}$ & & & & & & \\
\hline Immig Status=4 & 0.935 & 1.113 & 0.840 & .401 & 2.546 & 0.287 & 22.574 \\
\hline Immig Status=3 & 0.911 & .230 & 3.954 & $<.001$ & 2.487 & 1.583 & 3.907 \\
\hline Immig Status $=2$ & 1.595 & .285 & 5.599 & $<.001$ & 4.928 & 2.819 & 8.615 \\
\hline Immig Status $=1$ & 0.266 & .096 & 2.782 & .005 & 1.304 & 1.082 & 1.573 \\
\hline Immig Status $=0$ & $0^{\mathrm{a}}$ & & & & & & \\
\hline Sem Hrs-Attmptd & -0.017 & .005 & -3.668 & $<.001$ & 0.984 & 0.975 & 0.992 \\
\hline Sem Hrs-Passed & 0.022 & .006 & 3.508 & $<.001$ & 1.022 & 1.010 & 1.035 \\
\hline $\begin{array}{l}\text { GM \# of Prev. } \\
\text { MAT0012-0020 } \\
\text { Attmpts at institutn }\end{array}$ & -0.430 & .078 & -5.518 & $<.001$ & 0.651 & 0.559 & 0.758 \\
\hline Instructor's Age GM & -0.014 & .005 & -3.138 & .002 & 0.986 & 0.977 & 0.995 \\
\hline$[\mathrm{EnrlFT}=1] *[\mathrm{ST}=2]$ & -0.228 & .251 & -0.907 & .364 & 0.796 & 0.486 & 1.303 \\
\hline$[$ EnrlFT $=1] *[\mathrm{ST}=1]$ & 0.195 & .159 & 1.221 & .222 & 1.215 & 0.889 & 1.661 \\
\hline$[$ EnrlFT $=1] *[\mathrm{ST}=0]$ & 0.372 & .130 & 2.856 & .004 & 1.450 & 1.124 & 1.872 \\
\hline$[$ EnrlFT $=0] *[\mathrm{ST}=2]$ & 0.233 & .204 & 1.144 & .253 & 1.263 & 0.847 & 1.883 \\
\hline$[$ EnrlFT $=0] *[\mathrm{ST}=1]$ & 0.140 & .129 & 1.082 & .279 & 1.150 & 0.893 & 1.480 \\
\hline$[$ EnrlFT $=0] *[\mathrm{ST}=0]$ & $0^{\mathrm{a}}$ & & & & & & \\
\hline $\begin{array}{l}\text { Probability distribution: } B \\
\text { a. This coefficient is set to zero } b\end{array}$ & $\begin{array}{l}\text { inomial } \\
\text { ecause it is re }\end{array}$ & $\begin{array}{l}\text { Link fu } \\
\text { edundant. }\end{array}$ & tion: $\log$ & & & & \\
\hline Model's Overall Predictiv & Accuracy & Rate $=65$ & & & & & \\
\hline & & Predicted & & & & & \\
\hline $\begin{array}{l}\text { Observed } \\
\text { Success } \\
\text { Failure }\end{array}$ & $\begin{array}{l}\frac{\text { Success }}{\%(f=649} \\
\frac{1}{\%(f=355}\end{array}$ & & $\begin{array}{l}\text { Failure } \\
34.1 \%(f= \\
.0 \%(f=\end{array}$ & $\frac{765)}{1,511)}$ & & & \\
\hline
\end{tabular}


Five observations about this model are in order. First, the last among the eight student-level covariates merits some elaboration. It was computed in two steps: (a) first determining the total number of MAT0012 Pre-Algebra and/or MAT0020 Combined PreAlgebra/Elementary Algebra course attempts each student had at the institution prior to the Fall-2001 semester; and then (b) grand-mean centering that value. Therefore, the exponentiated coefficient value (0.651) indicates that, when all other covariate values are fixed, the likelihood of success in MAT0024 decreases by approximately $34.9 \%$ for each additional previous attempt at MAT0012/0020.

Second, the table reveals that men are a higher risk group than women, as evidenced by the exponentiated coefficient value for sex (1.377). It indicates that, according to this model, a female student's odds of success in MAT0024 are approximately $38 \%$ higher than that of a male student, when all other covariates are held constant.

Third, at first glance, one might think that the exponentiated coefficient for Black/African-American race (0.642) indicates that the Black students' odds of success in MAT0024 are about 35.8\% lower than non-Black students, when controlling for all other IVs. While this statistical interpretation is correct, it is important to not lose sight of the high unreported-value (and low internal-consistency) rate of the race variable. Said differently, there was no way to ascertain how many of the 859 missing values for race were Black students.

Fourth, it initially seemed counterintuitive to find that students who previously attempted an SLS study/life-skills course were estimated to have a $28.5 \%$ decrease in their odds of MAT0024 success compared to those who had not attempted such a course. 
However, it is important to remember that the typical student who enrolls in such an SLS courses is often more at-risk in terms of academic background than the general population of MAT0024 Elementary Algebra enrollees; for example, it is likely that SLS students, on average, have a higher likelihood of having placed into remedial-level coursework in other academic areas such as reading and writing.

Fifth and finally, although the IV of primary interest (seat-time level) was not found to be a significant predictor of MAT0024 course success, Table 17 shows that a cross-level interaction effect between seat time and whether the student's enrollment status was full-time in the Fall-2001 semester (denoted by EnrlFT=1) or not (denoted by EnrolFT $=0$ ) was detected. However, this interaction effect was limited in that statistical significance $(\mathrm{p}<.01)$ was obtained at only one level; namely, full-time students enrolled in short seat-time class sections. The final GLMM model's exponentiated coefficient value (1.450) indicates that the odds of MAT0024 success were estimated to be approximately $45 \%$ higher for a full-time student enrolled in a short seat-time class section than one who is not, when all other covariates are held constant.

\section{Analyses for Research Question 2}

This study's second research question was: In community-college developmentallevel algebra courses, is discrete survival time, as measured by date of withdrawal from the course, related to the number of class meetings per week and the duration of each meeting?

\section{Preliminary Descriptive Analyses}

Prior to obtaining the results of the survival analysis, descriptive statistics pertaining to student course-withdrawal frequencies and survival times were calculated. 
Disaggregated by seat-time level and by course session type, these descriptive statistics are summarized in Table 18.

Table 18

Cross-Tabulation of $W \&$ non-W Grades by Session \& Seat Time

\begin{tabular}{|c|c|c|c|c|c|c|}
\hline \multirow{3}{*}{\multicolumn{2}{|c|}{ Disaggregated by: }} & \multicolumn{4}{|c|}{ Course Grade $=\mathrm{W} ?$} & \multirow[b]{3}{*}{ Total } \\
\hline & & \multicolumn{2}{|c|}{ No } & \multicolumn{2}{|c|}{ Yes } & \\
\hline & & $n$ & $(\%)$ & $n$ & $(\%)$ & \\
\hline \multirow{3}{*}{$\begin{array}{l}\text { Session } \\
\text { Type }\end{array}$} & Session I (16-week) & 2,233 & $(82.7)$ & 467 & $(17.3)$ & 2,700 \\
\hline & Session III (12-week) & 444 & $(78.4)$ & 122 & $(21.6)$ & 566 \\
\hline & Session IV (8-week) & $13^{*}$ & $(72.2)^{*}$ & $5^{*}$ & $(27.8)^{*}$ & $18^{*}$ \\
\hline \multirow{3}{*}{$\begin{array}{l}\text { Seat-Time } \\
\text { Level }\end{array}$} & Short $($ Code $=0)$ & 926 & $(82.8)$ & 192 & $(17.2)$ & 1,118 \\
\hline & Medium $($ Code $=1)$ & 1,365 & $(82.1)$ & 297 & $(17.9)$ & 1,662 \\
\hline & Long $($ Code $=2)$ & 399 & $(79.2)$ & 105 & $(20.8)$ & 504 \\
\hline \multicolumn{2}{|c|}{ Aggregated Totals } & 2,690 & $(81.9)$ & 594 & $(18.1)$ & 3,284 \\
\hline
\end{tabular}

The aggregated totals reveal that, on average, approximately two out of every 11 (i.e., $18.1 \%$ of) MAT0024 enrollees received the withdrawal (W) grade. In terms of seat-time level, the course-withdrawal (W grade) rate was slightly under $21 \%$ for students in long seat-time classes. In contrast, it was under $18 \%$ for students enrolled in classes at the medium and short seat-time levels $-17.9 \%$ and $17.2 \%$, respectively. With regard to the disaggregation by class session length, it is noteworthy that the student rate of $\mathrm{W}$ grades is more than 4\% lower for those who were enrolled in the 99 full-semester Session I (16week) classes than for those in the 20 mini-term Session III classes. This difference merits further discussion in Chapter 5.

To ascertain whether these relative frequency differences were statistically significant, two chi-square tests for homogeneity of proportions were performed. For any test that yielded a significant $\chi^{2}$ test-statistic value (at the $\alpha=.05$ significance level), 
Hinkle, Wiersma, and Jurs (2003) suggest the absolute value of each cell's standardized residuals be compared against 2.00 in order to identify any major contributors to the $\chi^{2}$ value.

The first of these two nonparametric tests was a cross-tabular comparison of the variables $W$-grade $(0=\mathrm{No}, 1=\mathrm{Yes})$ and seat-time duration $(0=$ short, $1=$ medium, $2=\mathrm{long})$. This test did not reveal a statistically significant difference $\left(\chi^{2}=3.248, d f=2, p=.197\right)$. However, it is important to recognize that, unlike survival-analysis methods, this type of analysis is more superficial, in that it is limited to whether (or not) a student was awarded a withdrawal (W) grade. That is to say, it is incapable of quantifying group differences in when students withdrew (i.e., differences in time elapsed until the survival event was experienced).

The second of these two tests was a cross-tabular comparison of the variables $W$ grade $(0=\mathrm{No} ; 1=\mathrm{Yes})$ and session-length $(1=16$-week Session I class; $3=12$-week Session III class; 4=8-week Session IV class). This test did reveal a statistically significant difference $\left(\chi^{2}=6.874, d f=2, p=.032\right)$. Because $p<.05$, the absolute value of each cell's standardized residuals was then compared against 2.00 in order to identify any major contributors to the $\chi^{2}$ value. None of the six cells exhibited a standard residual of that magnitude. Specifically, all but one of the standardized residuals had absolute values no greater than one standard deviation; the sole exception was the cell containing the Session 3 students who received a "W" grade, for which the standardized residual value equaled 1.9. To further check the extent, if any, to which the comparatively low frequencies of the 18 students enrolled in the one Session IV class may have skewed the $\chi^{2}$ value, the chi-square test was re-run but with the 18 Session IV students omitted (i.e., 
with a sample of $N=3,266$, instead of $N=3,284)$. The difference in outcome $\left(\chi^{2}=5.740\right.$, $d f=1, p=.017$ ) was negligible.

\section{Construction of DTSA Life Table}

Singer and Willett (2003) describe the life table as "the primary tool for describing event occurrence data" (p. 325). A life table subdivides the study's time period into subintervals and summarizes the interval-to-interval change of the event histories for the entire set of subjects in the sample. For each interval within the study's time period, the life table provides "... the cumulative proportion of subjects that survive to the beginning of that interval (the survival rate), and how many subjects who enter the interval experience the event before the midpoint of that interval (the hazard)" (Hox, 2010, p. 160).

The respective life tables for Fall-2001 MAT0024 cohort students within each seat-time level are shown in Tables 19, 20, and 21. Only the columns of primary importance are shown, although others (e.g., the probability density, the hazard rate, and their respective standard errors) contributed to the computation of the estimated survivor functions. 


\begin{tabular}{|c|c|c|c|c|c|c|c|c|}
\hline \multicolumn{9}{|c|}{ Life Table for Research Question 2 - Short Seat Time } \\
\hline & & \multicolumn{4}{|c|}{ Number of Students } & \multicolumn{3}{|c|}{ Proportion of Students } \\
\hline $\mathrm{Wk}$ & $\begin{array}{c}\text { Time } \\
\text { interval }\end{array}$ & $\begin{array}{c}\text { Entering } \\
\text { interval } \\
\end{array}$ & $\begin{array}{c}\text { Censored } \\
\text { during } \\
\text { interval } \\
\end{array}$ & $\begin{array}{c}\text { Exposed to } \\
\text { Course } \\
\text { Withdrawal } \\
\text { Risk } \\
\end{array}$ & $\begin{array}{c}\text { Withdrew } \\
\text { from } \\
\text { course } \\
\end{array}$ & $\begin{array}{l}\text { Withdrawn } \\
\text { from } \\
\text { course } \\
\text { within time } \\
\text { interval } \\
\text { [Hazard } \\
\text { Function] }\end{array}$ & $\begin{array}{l}\text { Surviving } \\
\text { at End of } \\
\text { Interval } \\
\text { (among at- } \\
\text { risk at start } \\
\text { of time } \\
\text { interval) } \\
\end{array}$ & $\begin{array}{c}\text { Surviving at } \\
\text { End of } \\
\text { Interval } \\
\text { (cumulative) } \\
\text { [Survivor } \\
\text { Function] }\end{array}$ \\
\hline 1 & $0-6$ & 1118 & 0 & 1118.0 & 0 & .00 & 1.00 & 1.00 \\
\hline 2 & $7-13$ & 1118 & 0 & 1118.0 & 1 & .00 & 1.00 & 1.00 \\
\hline 3 & $14-20$ & 1117 & 0 & 1117.0 & 4 & .00 & 1.00 & 1.00 \\
\hline 4 & $21-27$ & 1113 & 0 & 1113.0 & 4 & .00 & 1.00 & .99 \\
\hline 5 & $28-34$ & 1109 & 0 & 1109.0 & 5 & .00 & 1.00 & .99 \\
\hline 6 & $35-41$ & 1104 & 0 & 1104.0 & 8 & .01 & .99 & .98 \\
\hline 7 & $42-48$ & 1096 & 0 & 1096.0 & 11 & .01 & .99 & .97 \\
\hline 8 & $49-55$ & 1085 & 0 & 1085.0 & 18 & .02 & .98 & .95 \\
\hline 9 & $56-62$ & 1067 & 0 & 1067.0 & 24 & .02 & .98 & .93 \\
\hline 10 & $63-69$ & 1043 & 0 & 1043.0 & 68 & .07 & .93 & .87 \\
\hline 11 & $70-76$ & 975 & 0 & 975.0 & 0 & .00 & 1.00 & .87 \\
\hline 12 & $77-83$ & 975 & 0 & 975.0 & 0 & .00 & 1.00 & .87 \\
\hline 13 & $84-90$ & 975 & 0 & 975.0 & 1 & .00 & 1.00 & .87 \\
\hline 14 & $91-97$ & 974 & 0 & 974.0 & 0 & .00 & 1.00 & .87 \\
\hline 15 & $98-104$ & 974 & 0 & 974.0 & 0 & .00 & 1.00 & .87 \\
\hline 16 & $105-111$ & 974 & 0 & 974.0 & 0 & .00 & 1.00 & .87 \\
\hline 17 & 112-118 & 974 & 926 & 511.0 & 48 & .09 & .91 & .79 \\
\hline
\end{tabular}




\begin{tabular}{|c|c|c|c|c|c|c|c|c|}
\hline \multicolumn{9}{|c|}{ Life Table for Research Question 2 - Medium Seat Time } \\
\hline & & \multicolumn{4}{|c|}{ Number of Students } & \multicolumn{3}{|c|}{ Proportion of Students } \\
\hline $\mathrm{Wk}$ & $\begin{array}{c}\text { Time } \\
\text { interval }\end{array}$ & $\begin{array}{c}\text { Entering } \\
\text { interval } \\
\end{array}$ & $\begin{array}{c}\text { Censored } \\
\text { during } \\
\text { interval } \\
\end{array}$ & $\begin{array}{c}\text { Exposed to } \\
\text { Course } \\
\text { Withdrawal } \\
\text { Risk } \\
\end{array}$ & $\begin{array}{c}\text { Withdrew } \\
\text { from } \\
\text { course } \\
\end{array}$ & $\begin{array}{l}\text { Withdrawn } \\
\text { from } \\
\text { course } \\
\text { within time } \\
\text { interval } \\
\text { [Hazard } \\
\text { Function] }\end{array}$ & $\begin{array}{l}\text { Surviving } \\
\text { at End of } \\
\text { Interval } \\
\text { (among at- } \\
\text { risk at start } \\
\text { of time } \\
\text { interval) } \\
\end{array}$ & $\begin{array}{c}\text { Surviving at } \\
\text { End of } \\
\text { Interval } \\
\text { (cumulative) } \\
\text { [Survivor } \\
\text { Function] }\end{array}$ \\
\hline 1 & $0-6$ & 1662 & 0 & 1662.0 & 1 & .00 & 1.00 & 1.00 \\
\hline 2 & $7-13$ & 1661 & 0 & 1661.0 & 8 & .00 & 1.00 & .99 \\
\hline 3 & $14-20$ & 1653 & 0 & 1653.0 & 22 & .01 & .99 & .98 \\
\hline 4 & $21-27$ & 1631 & 0 & 1631.0 & 14 & .01 & .99 & .97 \\
\hline 5 & $28-34$ & 1617 & 0 & 1617.0 & 15 & .01 & .99 & .96 \\
\hline 6 & $35-41$ & 1602 & 0 & 1602.0 & 13 & .01 & .99 & .96 \\
\hline 7 & $42-48$ & 1589 & 0 & 1589.0 & 30 & .02 & .98 & .94 \\
\hline 8 & $49-55$ & 1559 & 0 & 1559.0 & 28 & .02 & .98 & .92 \\
\hline 9 & $56-62$ & 1531 & 0 & 1531.0 & 26 & .02 & .98 & .91 \\
\hline 10 & $63-69$ & 1505 & 0 & 1505.0 & 71 & .05 & .95 & .86 \\
\hline 11 & $70-76$ & 1434 & 0 & 1434.0 & 2 & .00 & 1.00 & .86 \\
\hline 12 & $77-83$ & 1432 & 0 & 1432.0 & 2 & .00 & 1.00 & .86 \\
\hline 13 & $84-90$ & 1430 & 0 & 1430.0 & 0 & .00 & 1.00 & .86 \\
\hline 14 & $91-97$ & 1430 & 0 & 1430.0 & 0 & .00 & 1.00 & .86 \\
\hline 15 & $98-104$ & 1430 & 0 & 1430.0 & 2 & .00 & 1.00 & .86 \\
\hline 16 & $105-111$ & 1428 & 0 & 1428.0 & 1 & .00 & 1.00 & .86 \\
\hline 17 & $112-118$ & 1427 & 1365 & 744.5 & 62 & .08 & .92 & .79 \\
\hline
\end{tabular}




\begin{tabular}{|c|c|c|c|c|c|c|c|c|}
\hline \multicolumn{9}{|c|}{ Life Table for Research Question 2 - Long Seat Time } \\
\hline & & \multicolumn{4}{|c|}{ Number of Students } & \multicolumn{3}{|c|}{ Proportion of Students } \\
\hline $\mathrm{Wk}$ & $\begin{array}{c}\text { Time } \\
\text { interval }\end{array}$ & $\begin{array}{c}\text { Entering } \\
\text { interval } \\
\end{array}$ & $\begin{array}{c}\text { Censored } \\
\text { during } \\
\text { interval } \\
\end{array}$ & $\begin{array}{c}\text { Exposed } \\
\text { to Course } \\
\text { Withdraw } \\
\text { al Risk }\end{array}$ & $\begin{array}{c}\text { Withdrew } \\
\text { from } \\
\text { course } \\
\end{array}$ & $\begin{array}{l}\text { Withdrawn } \\
\text { from } \\
\text { course } \\
\text { within time } \\
\text { interval } \\
\text { [Hazard } \\
\text { Function] }\end{array}$ & $\begin{array}{l}\text { Surviving } \\
\text { at End of } \\
\text { Interval } \\
\text { (among at- } \\
\text { risk at start } \\
\text { of time } \\
\text { interval) } \\
\end{array}$ & $\begin{array}{c}\text { Surviving at } \\
\text { End of } \\
\text { Interval } \\
\text { (cumulative) } \\
\text { [Survivor } \\
\text { Function] }\end{array}$ \\
\hline 1 & $0-6$ & 504 & 0 & 504.0 & 2 & .00 & 1.00 & 1.00 \\
\hline 2 & $7-13$ & 502 & 0 & 502.0 & 6 & .01 & .99 & .98 \\
\hline 3 & $14-20$ & 496 & 0 & 496.0 & 3 & .01 & .99 & .98 \\
\hline 4 & $21-27$ & 493 & 0 & 493.0 & 11 & .02 & .98 & .96 \\
\hline 5 & $28-34$ & 482 & 0 & 482.0 & 11 & .02 & .98 & .93 \\
\hline 6 & $35-41$ & 471 & 0 & 471.0 & 10 & .02 & .98 & .91 \\
\hline 7 & $42-48$ & 461 & 0 & 461.0 & 1 & .00 & 1.00 & .91 \\
\hline 8 & $49-55$ & 460 & 0 & 460.0 & 5 & .01 & .99 & .90 \\
\hline 9 & $56-62$ & 455 & 0 & 455.0 & 6 & .01 & .99 & .89 \\
\hline 10 & $63-69$ & 449 & 0 & 449.0 & 22 & .05 & .95 & .85 \\
\hline 11 & $70-76$ & 427 & 0 & 427.0 & 0 & .00 & 1.00 & .85 \\
\hline 12 & $77-83$ & 427 & 0 & 427.0 & 0 & .00 & 1.00 & .85 \\
\hline 13 & $84-90$ & 427 & 0 & 427.0 & 0 & .00 & 1.00 & .85 \\
\hline 14 & $91-97$ & 427 & 0 & 427.0 & 0 & .00 & 1.00 & .85 \\
\hline 15 & $98-104$ & 427 & 0 & 427.0 & 0 & .00 & 1.00 & .85 \\
\hline 16 & $105-111$ & 427 & 0 & 427.0 & 0 & .00 & 1.00 & .85 \\
\hline 17 & $112-118$ & 427 & 399 & 227.5 & 28 & .12 & .88 & .74 \\
\hline
\end{tabular}

In each of these three tables, the semester-long (118-day) tracking period was grouped into week-to-week sub-intervals. It should be noted that some student subjects had course survival (i.e., course withdrawal) times that were not whole numbers; namely, some of the Session III and Session IV students had survival times that contained a fractional (decimal) portion, due to the proration adjustment discussed earlier. In light of this, one might ask: where in the life table would a Session III student who, for example, 
had a prorated survival time of 27.53 days (i.e., $21 \times 118 / 90$ ) appear in Table 20 (regarding medium seat-time level students)? In other words, would 27.53 days be truncated (to 27 ) or rounded (to 28)? The answer is: that student was included among the 14 students who withdrew in the fourth week, which is shown in that table as time interval 21-27 days. So, in actuality, that interval reflects unrounded course survival times that are at least 21.0 days in duration but are less than 28.0 days. Said differently, each time interval may be viewed as encompassing all the truncated hazard-event times (i.e., time when assigned a W grade) that transpired within its left and right boundaries.

The two most important columns in these life tables are those that empirically estimate the survivor function and the hazard function.

The survivor function reflects the cumulative proportion of students in the $i^{\text {th }}$ time interval who are still surviving (i.e., have not yet been awarded a course-withdrawal grade of $\mathrm{W})$ at the beginning of the $(i+1)^{\mathrm{st}}$ time interval. Said differently, the survivor function provides the probability that a randomly selected student will survive past the $i^{\text {th }}$ time interval. For example, in the life table for medium seat-time students (Table 20), there were a total of 31 students (i.e., $1+8+22$ ) who withdrew within the first three weeks (i.e., when $i=3$ ). This meant that, by the beginning of the fourth week (when $i+1=4), 1631$ of the original 1662 medium-seat-time students were still surviving; that is, only 31 had experienced the hazard event of course withdrawal. Consequently, the survivor function's value in that third week equaled the proportion $1631 / 1662 \approx .98$, which represents the fact that, as of the beginning of the fourth week (i.e., the start of the 28 th day of the full semester), approximately $98 \%$ of all medium seat-time student enrollees were still actively enrolled in their MAT0024 Elementary Algebra course. In probability terms, one 
might express this as approximately a $98 \%$ likelihood that a randomly selected medium seat-time student will survive - namely, not experience the hazard of official course withdrawal with a grade of $\mathrm{W}$ - until at least the beginning of the fourth week of the full semester.

The hazard function quantifies, as its name aptly suggests, the probability that a subject who had not experienced the hazard event in any previously occurring time subinterval will experience the hazard in the current sub-interval. It differs from the survivor function in three major ways. First, and most obviously, the hazard function is concerned with the rate of event (hazard) occurrence, whereas the survivor function pertains to the rate of event nonoccurrence. Second, unlike the survivor function, the hazard function is not calculated in a cumulative (nor total-time-elapsed) manner; instead, it provides an interval-centric probability. Thirdly, while the calculation of the survivor function relies upon one unchanging denominator value (namely, the total number of subjects), the hazard function is a conditional probability; that is to say, its denominator (i.e., the size of its possible risk set) is based on the number of remaining survivors at the beginning of a given time sub-interval.

To more clearly illustrate this, consider the eighth week of the life table for the short seat-time students (Table 19). By the beginning of that time interval (i.e., the start of the $49^{\text {th }}$ day), there had already been 33 students who experienced the hazard event, the assignment of a course grade of W. Hence, there were 1,085 students remaining among the original 1,118 short-seat-time students. The hazard function value, therefore, was the conditional probability that a student would experience the hazard event in that eighth week, given that the student had not experienced the hazard event in any of the preceding 
weeks. Since 18 of the remaining 1,085 students were assigned a W grade in that eighth week, the hazard function value in that time sub-interval equaled $18 / 1085 \approx .0166 \approx .02$.

The tabular form (in Tables 19-21) of presenting the survivor and hazard functions is especially valuable in that it reveals, at the micro level, subinterval-tosubinterval changes in event occurrence and nonoccurrence. Its primary drawback, however, is the labor required to discern macroscopic trends across a study's entire timespan. A simpler, clearer way to discern group differences in survival rates-and, implicitly, hazard rates as well—across a study's time spectrum is by viewing a graph that plots (and overlays) each group's estimated survivor function.

Figure 1 displays the graphs of the estimated survivor functions that were initially shown in Tables 19-21. The graph was constructed with the same seven-day (week-toweek) time-interval groupings utilized in the construction of the life tables, as evidenced by the descending stair-step shape of these three survivor functions. One can see that, during the first two weeks of the semester, all three functions had survivor rates at or near $100 \%$ (1.00), since very few students withdrew from their MAT0024 class so early in the academic term. Soon thereafter, however, three non-intersecting trend paths began to develop. Specifically, the short (i.e., the solid line) and the long (i.e., the dash-dash line) seat-time students displayed the highest and lowest week-to-week survivor rates, respectively. Also, as anticipated from the high hazard-function rates in the tenth week (days 63-69) and seventeenth week (days 112-118) that were shown in Tables 19-21, all three survivor-function graphs experienced their most rapid descent in those two time sub-intervals. 


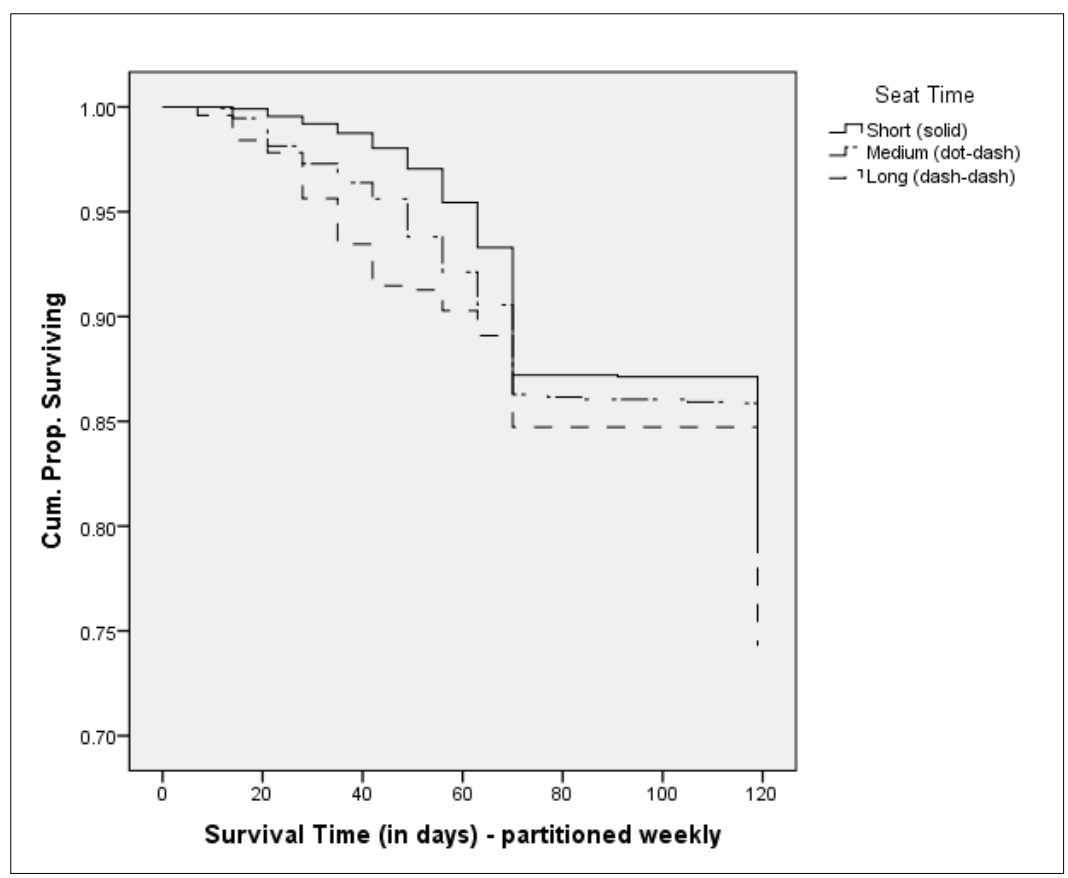

Figure 1. Graph of Q2's estimated survivor functions (weekly partitioning).

As much as Figure 1 enhances our perspective of cumulative changes in student retention (survival) rates on a period-to-period basis, some prefer a graphical plot that explicitly reveals changes in student attrition (cumulative hazard). Figure 2 provides such a graph, by displaying the ratio of students who had withdrawn across the 118-day semester period. For example, one can see in Figure 2 that after the student-initiated withdrawal deadline (at the $60 \%$ point of the semester, which was on the $69^{\text {th }}$ day of the Session I semester) the short seat-time level had the lowest attrition rate $(\approx 13 \%)$, while the long seat-time level had the highest attrition rate $(\approx 15 \%)$. Said differently, Figure 1 and Table 19 affirm that, on the $69^{\text {th }}$ day and for several weeks thereafter, the short seattime level had the highest retention rate $(\approx 87 \%)$, whereas Figure 2 (and Table 21 ) confirm that the long seat-time level's retention rate was approximately $85 \%$.

However, before one prematurely asks whether there was a statistically significant difference between these seat-time groups' attrition/retention rates, it is important to not 
lose sight of the fact that course withdrawals did not entirely cease after the passing of the student-initiated withdrawal deadline. As previously discussed, there were instructorinputted course withdrawals that transpired throughout the entire semester, most visibly at the very end of the 118-day term. That fact is made evident in two ways: (a) numerically, by revisiting the $16^{\text {th }}$ and $17^{\text {th }}$ weeks of the three life tables (Tables 19-21), during which there was a large decrease in estimated survivor function values; and (b) graphically, by observing the large descent on day 118 in the survivor function values (Figure 1), which, of course, is identical to the identically large ascent in cumulative hazard (attrition) rates shown in Figure 2. Certainly, because the overlaid graphs obscure one another at the end of the 118-day time period, the numerical/tabular view (in Tables 19-21) is a more informative means by which to compare end-of-semester group survivor rates. These tables show an approximately $5 \%$ difference in estimated survivor-function values between the long seat-time group (74\%) versus that of the short and medium groups (both 79\%). 


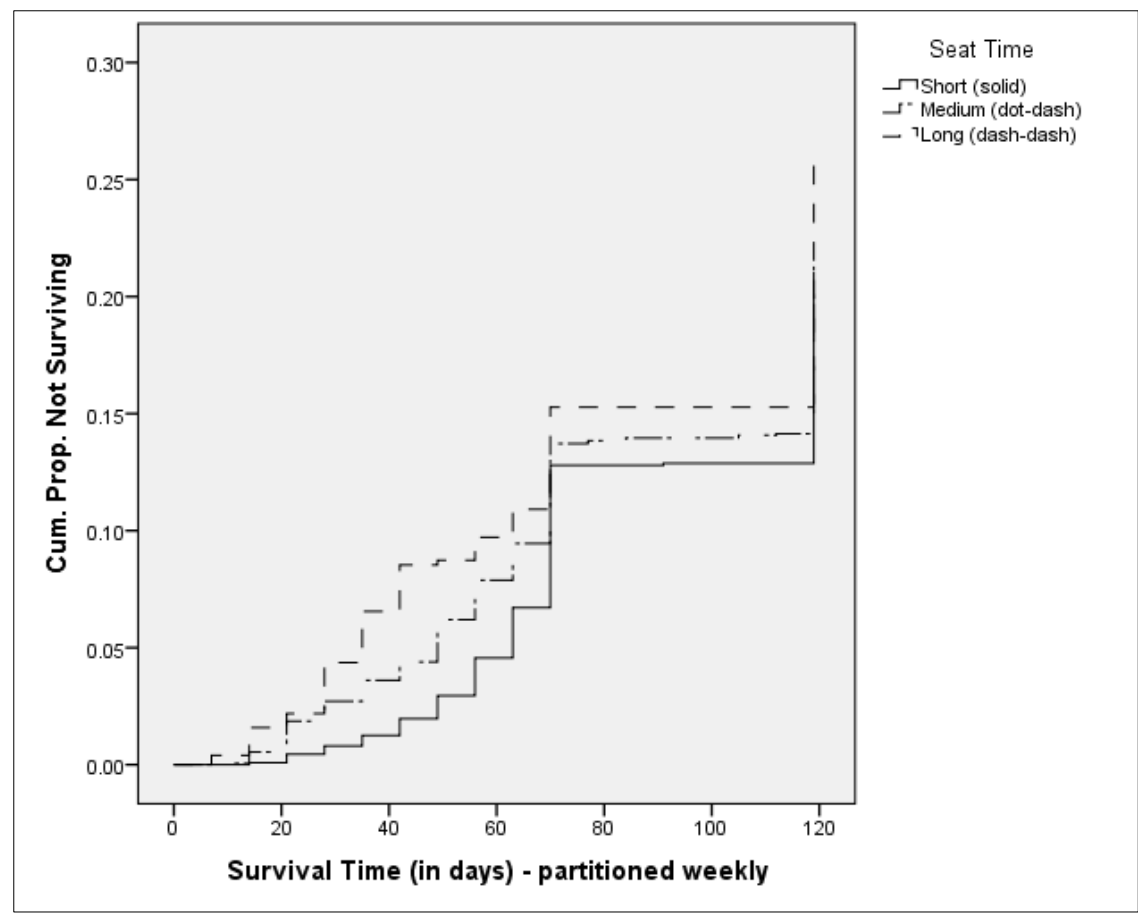

Figure 2. Graph of Q2's estimated non-survivor (cumulative-hazard) functions.

Before concluding this discussion of the tabular and graphical displays of each seat-time level's estimated survivor function, it is important to acknowledge the benefits and the potential pitfalls of having subdivided this survival analysis's 118-day time period into 17 weekly segments. In Tables 19-21 and Figures 1-2, there were two considerations that warranted doing so. First, when constructing a life table, Singer and Willett (2003) recommend selecting “... the temporal partition most relevant for [one's] chosen time metric and for the way in which events unfold" (p. 328). College administrators, faculty, and students routinely think of academic semester in terms of weeks, not days. Second, for a life table to provide a sufficiently informative — but not an overwhelmingly lengthy and, hence, unreadable - historical event-occurrence timeline, a partitioning of the semester into 17 (weekly) encapsulations is preferable to118 (daily) snapshots. At the same time, though, care must be taken that the chosen partition did not affect the 
statistical analysis and, moreover, did not provide a misleading graphical view of coursewithdrawal events. To help alleviate that concern, Figure 3 below provides an unpartitioned (day-to-day) view of each seat-time level's estimated survivor function. A cursory comparison of this figure with the previously shown Figure 1 confirms that the two approaches, weekly/partitioned versus daily/un-partitioned, provide consistent eventhistory portrayals.

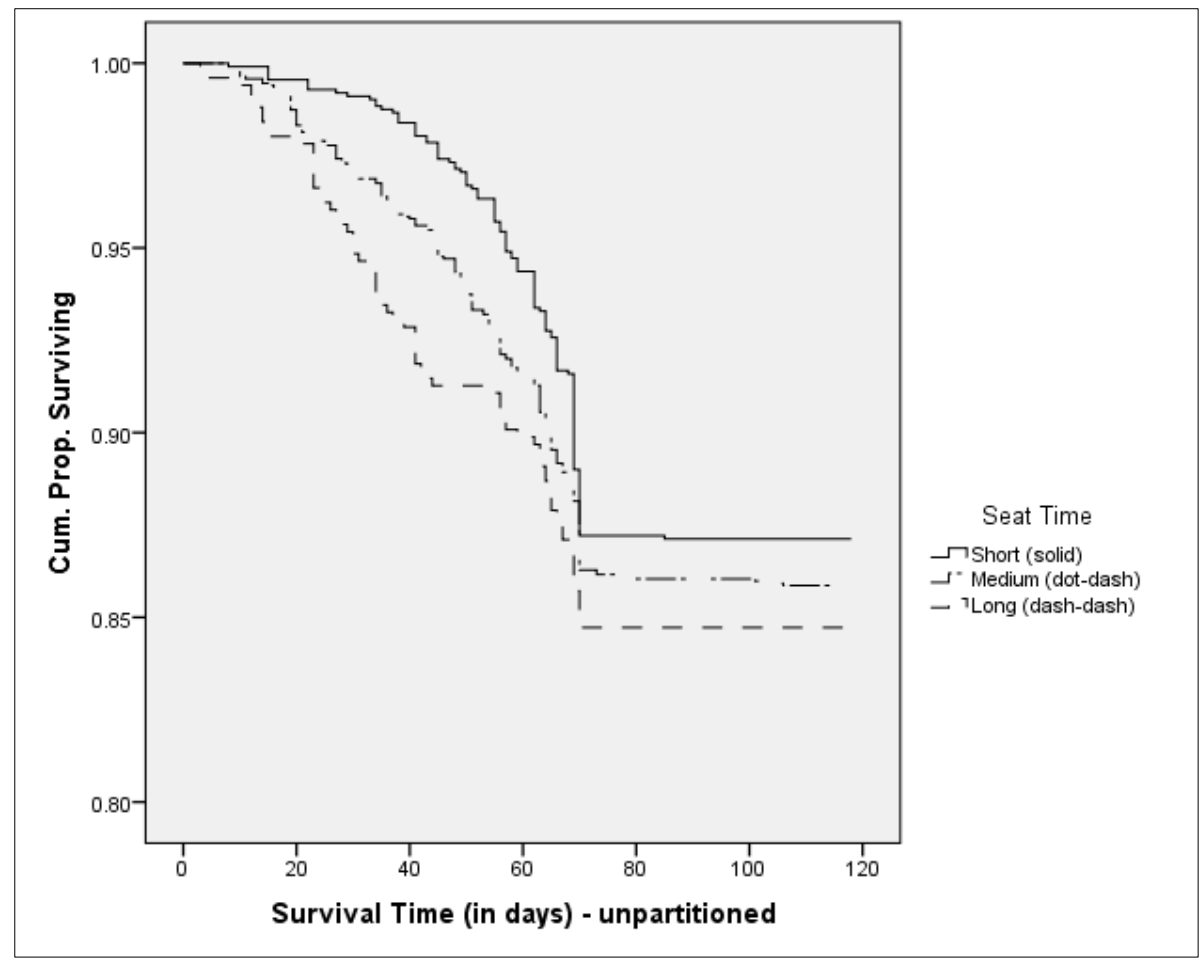

Figure 3. Graph of Q2's estimated survivor functions (un-partitioned).

\section{Statistical Tests for Group Differences}

While the life table for each seat-time level and its accompanying estimated survivor-function exhibits numerically and graphically a visually detectable difference between groups, that is, of course, not a statistically sufficient condition upon which to infer that significant group differences exist in the population. To achieve that end, there are two inferential methods for life-table estimation and group-difference testing: the 
actuarial approach and the product-limit (also known as the Kaplan-Meier) method (Tabachnick \& Fidell, 2007).

Actuarial life-table approach with Wilcoxon (Gehan) test. The actuarial approach was used in the creation of the previously discussed life tables (shown in Tables 19-21) and their corresponding survivor-function graphs (Figure 1). Hosmer, Lemeshow, and May (2008) point out that dating back to the 1960's:

A number of statistical tests have been proposed... and most software packages provide results from at least two of these tests. However, comparison of the results obtained by different packages can become confusing due to small but annoying differences in terminology and [calculation] methods... The original developers of these tests sought ways to extend tests used with non-censored data to the censored data setting. (p. 45)

For its actuarial method's hypothesis test, SPSS version 20 relies upon the Wilcoxon (Gehan) test, which some refer to as the generalized Wilcoxon test or the Gehan-Breslow test (Hosmer et al., 2008). The Wilcoxon (Gehan) test statistic is computed in a way that is not affected in any way by partitioning or non-partitioning of the life table's time period, which the researcher confirmed by running the test in both manners.

For comparisons that involve three or more groups, SPSS enables one to test for two types of differences: overall and pairwise. When the overall option was selected, the Wilcoxon (Gehan) test-statistic value $\left(\chi^{2}=4.412, d f=2, p=.110\right)$ was not statistically significant. In contrast, Table 22 shows that the pairwise option revealed a statistically significant difference $(p<.05)$ in survival rates between the short and long seat-time levels, which were coded 0 and 2, respectively. Neither the short nor long seat-time 
level's survival rates were found to be significantly different than that of the medium level (coded 1).

Table 22

Results of Actuarial-Method Hypothesis Test for Research Question $2^{a}$ Wilcoxon

\begin{tabular}{ccccc} 
Seat Time & Seat Time & $\left(\right.$ Gehan) $\chi^{2}$ & $d f$ & Sig. \\
\hline \multirow{2}{*}{$0^{*}$} & 1 & 0.678 & 1 & .410 \\
& $2 *$ & 4.377 & 1 & .036 \\
\hline \multirow{2}{*}{1} & 0 & 0.678 & 1 & .410 \\
& 2 & 2.476 & 1 & .116 \\
\hline \multirow{2}{*}{$2^{*}$} & $0^{*}$ & 4.377 & 1 & .036 \\
& 1 & 2.476 & 1 & .116 \\
\hline
\end{tabular}

${ }^{\text {a. }}$ Comparisons are exact.

* $p<.05$

Confirmatory finding via the product-limit (Kaplan-Meier) method. The Kaplan-Meier (KM) method is an extension of the actuarial (life-table) approach in that it computes survival statistics after every hazard event occurrence, instead of grouping rounded survival times within fixed time interval widths. This minimizes the likelihood of ties in hazard-event times (Hosmer et al., 2008; Hox, 2010; Singer \& Willett, 2003; Tabachnick \& Fidell, 2007).

When survival-analysis data sets are such that ties were frequent and unavoidable, as was the case with this dissertation study, Hosmer et al. (2008) suggest that other discrete-time models may be more appropriate than the KM method. Nonetheless, since the KM method yields comparatively the "most refined" estimate of the survivor-function (Singer \& Willett, 2003, p. 486), investigating it seemed warranted and worthwhile. Table 23 shows the results of SPSS's Kaplan-Meier survival-analysis algorithm: 
Table 23

Results of Kaplan-Meier Method Pairwise Comparisons

\begin{tabular}{llcccccc}
\hline & & \multicolumn{2}{c}{ Short } & \multicolumn{3}{c}{ Medium } & \multicolumn{2}{c}{ Long } \\
\cline { 3 - 7 } Test & Seat Time & $\chi^{2}$ & Sig. & $\chi^{2}$ & Sig. & $\chi^{2}$ & Sig. \\
\hline Log Rank & Short & & & 0.443 & .506 & 3.805 & .051 \\
(Mantel-Cox) & Medium & 0.443 & .506 & & & 2.351 & .125 \\
& Long & 3.805 & .051 & 2.351 & .125 & & \\
\hline Breslow & Short & & & 0.675 & .411 & $4.447^{*}$ & .035 \\
(Generalized & Medium & 0.675 & .411 & & & 2.501 & .114 \\
Wilcoxon) & Long & $4.447^{*}$ & .035 & 2.501 & .114 & & \\
\hline Tarone-Ware & Short & & & 0.552 & .457 & $4.117^{*}$ & .042 \\
& Medium & 0.552 & .457 & & & 2.425 & .119 \\
& Long & $4.117^{*}$ & .042 & 2.425 & .119 & & \\
\hline
\end{tabular}

$* p<.05$

The Tarone-Ware $\left(\chi^{2}=4.117, d f=1, p=.042\right)$ and Breslow's generalized-Wilcoxon $\left(\chi^{2}=\right.$ 4.447, $d f=1, p=.035)$ tests yielded results very similar to the actuarial method's Wilcoxon (Gehan) test-statistic value $\left(\chi^{2}=4.377, d f=1, p=.036\right)$. That is to say, all three displayed a significant difference between the short and long seat-time levels' respective estimated survivor functions, all with roughly equivalent $p$-values.

One might question why the log rank (Mantel-Cox) test $\left(\chi^{2}=3.805, d f=1, p=\right.$ .051) rendered a contrary finding. This was attributable to differences among these three test statistics in how time points are treated. According to SPSS's help menu, the log-rank (Mantel-Cox) test weights all time points equally; the Breslow (generalized Wilcoxon) test assigns weights based upon the number of at-risk cases remaining at each time point; and the Tarone-Ware test weights by the square-root of the number of at-risk cases remaining at each time point. Consequently, as Hosmer et al. (2008) point out, the logrank test tends to place greater emphasis on group differences between survivor-functions at later time points in the study period, whereas the generalized Wilcoxon test assigns 
more weight on differences between the survival functions at earlier time points. Tests like Tarone-Ware "use weight functions intermediate between these" (p. 48). Therefore, in light of the $60 \%$-point, student-initiated withdrawal deadline discussed previously, the log-rank test's equal weighting of time points was the least appropriate for this time-tocourse-withdrawal data set; hence, its contrary finding may be disregarded.

\section{Analyses for Research Question 3}

This study's third research question was: Across the sequence of communitycollege algebra courses, is there a longitudinal difference in the time to complete at least one general-education college-level mathematics course between students who satisfied the prerequisite algebra course requirements by completing one or more algebra courses scheduled in a one-day-per-week, longer-duration format and students whose prerequisite algebra course requirements were entirely fulfilled in courses that had medium-duration (twice weekly) or shorter-duration (thrice weekly) class meetings?

\section{Descriptive Summaries of Cohort's Longitudinal Case Histories}

Among the Fall-2001 MAT0024 Elementary Algebra student cohort $(N=3,284)$, slightly fewer than two out of three $(n=2,127 ; 64.8 \%)$ subsequently went on to attempt one or more mathematics courses at the college during the remaining five semesters of the six-semester longitudinal tracking period. One might consider that relative frequency value of $64.8 \%$ as an aggregated way to quantify the probability of a randomly selected student's mathematics-course persistence beyond the initial (Fall-2001) semester of the tracking period.

Table 24 displays the differences in this persistence measure among the three levels of Fall-2001 MAT0024 seat-time duration: 
Table 24

Cross-Tabulation by Seat-Time Level of Subsequent Course Attempt

\begin{tabular}{|c|c|c|c|c|c|c|c|c|}
\hline \multirow{3}{*}{$\begin{array}{c}\text { At Least One } \\
\text { Subsequent Math } \\
\text { Course Attempt? }\end{array}$} & \multicolumn{6}{|c|}{ Seat Time } & \multirow{2}{*}{\multicolumn{2}{|c|}{ Total }} \\
\hline & \multicolumn{2}{|c|}{ Short } & \multicolumn{2}{|c|}{ Medium } & \multicolumn{2}{|c|}{ Long } & & \\
\hline & $n$ & $(\%)$ & $n$ & $(\%)$ & $n$ & $(\%)$ & $n$ & $(\%)$ \\
\hline No & 333 & $(29.8)$ & 605 & $(36.4)$ & 219 & $(43.5)$ & 1,157 & $(35.2)$ \\
\hline Yes & 785 & $(70.2)$ & 1,057 & $(63.6)$ & 285 & $(56.5)$ & 2,127 & $(64.8)$ \\
\hline Total & 1,118 & $(34.0)$ & 1,662 & $(50.6)$ & 504 & $(15.3)$ & 3,284 & $(100.0)$ \\
\hline
\end{tabular}

The short seat-time level displayed (at 70.2\%) the highest rate of persistence; that is to say, on average, more than 7 out of 10 of students who were enrolled in a short seat-time section of MAT0024 in the Fall-2001 semester went on to re-enroll at the same institution in a mathematics course at least once over the span of the next five semesters. The long seat-time level students had the lowest rate $(56.5 \%)$ of re-enrollment persistence. To determine whether the differences in these persistence rates were statistically significant or more likely attributable to chance fluctuations, a chi-square test for homogeneity of proportions was performed. It yielded a statistically significant difference $\left(\chi^{2}=30.457, d f\right.$ $=2, p<.001)$. This confirmed that students who had enrolled in a short MAT0024 class were more likely to enroll in at least one additional mathematics course at the same college during the subsequent five semesters than, say, students in a long MAT0024 class.

The enrollment patterns of the 2,127 persisters were then examined on a semester-by-semester manner. Nearly $80 \%(n=1,698)$ of these 2,127 persisters enrolled in a mathematics course during the Winter-2002 semester; said differently, these students avoided a semester-long or lengthier gap before re-attempting another mathematics course after their Fall-2001 enrollment in MAT0024. Further disaggregation of these 
1,698 Winter-2002 students by seat-time level revealed that the short seat-time level cohort students had the highest relative rate of re-enrollment (624 out of 1,117, or $55.9 \%$ ), while the long seat-time level students had the lowest (197 out of 505, or 39.0\%).

The cohort students' aggregated enrollment frequencies were then tabulated on a semester-to-semester basis. Among the 2,127 persisters within the Fall-2001 MAT0024 cohort $(N=3,284)$, it was found that: $562(26.4 \%)$ enrolled in a Summer-2002

mathematics course at this same institution; 1,048 (49.3\%) in a Fall-2002 course; 787 $(37.0 \%)$ in a Winter-2003 course; and $356(16.7 \%)$ in a Summer-2003 course.

Each student's total number of mathematics course attempts during the sixsemester tracking period was then tabulated. Table 25 summarizes those tabulations, in both a seat-time-disaggregated and full-cohort-aggregated manner.

Table 25

Number of Course Attempts Within Longitudinal Tracking Period

\begin{tabular}{|c|c|c|c|c|c|c|c|c|}
\hline \multirow{3}{*}{$\begin{array}{c}\text { \# of Course } \\
\text { Attempts w/in } \\
\text { Tracking Period }\end{array}$} & \multicolumn{6}{|c|}{ Disaggregated by Seat Time } & \multirow{2}{*}{\multicolumn{2}{|c|}{$\begin{array}{c}\text { Aggregated } \\
\text { Total }\end{array}$}} \\
\hline & \multicolumn{2}{|c|}{ Short } & \multicolumn{2}{|c|}{ Medium } & \multicolumn{2}{|c|}{ Long } & & \\
\hline & $n$ & $(\%)$ & $n$ & $(\%)$ & $n$ & $(\%)$ & $n$ & $(\%)$ \\
\hline 1 & 333 & (29.8) & 605 & (36.4) & 219 & (43.5) & 1,157 & $(35.2)$ \\
\hline 2 & 280 & $(25.0)$ & 453 & (27.3) & 131 & $(26.0)$ & 864 & $(26.3)$ \\
\hline 3 & 215 & $(19.2)$ & 279 & $(16.8)$ & 76 & $(15.1)$ & 570 & $(17.4)$ \\
\hline 4 & 173 & $(15.5)$ & 190 & (11.4) & 45 & $(8.9)$ & 408 & (12.4) \\
\hline 5 & 79 & (7.1) & 91 & $(5.5)$ & 22 & (4.4) & 192 & $(5.8)$ \\
\hline 6 & 27 & (2.4) & 31 & (1.9) & 10 & $(2.0)$ & 68 & (2.1) \\
\hline 7 & 10 & (0.9) & 10 & $(0.6)$ & 1 & $(0.2)$ & 21 & $(0.6)$ \\
\hline 8 & 1 & $(0.1)$ & 2 & $(0.1)$ & 0 & $(0.0)$ & 3 & $(0.1)$ \\
\hline 9 & 0 & $(0.0)$ & 1 & $(0.1)$ & 0 & $(0.0)$ & 1 & $(0.0)$ \\
\hline Total & 1,118 & $(100.0)$ & 1,662 & $(100.0)$ & 504 & $(100.0)$ & 3,284 & $(100.0)$ \\
\hline
\end{tabular}

${ }^{a}$ This column is inclusive of the Fall-2001 MAT0024 course attempt.

To better illustrate, by example, the utility of this table, consider cohort students who had at least four course attempts, including their Fall-2001 MAT0024 enrollment. In the 
aggregate, there were 693 such students (i.e., $408+192+68+21+3+1$ ), which equated to $21.1 \%$ of the 3,284 members of the cohort. When disaggregated by seat-time level, one finds that $25.9 \%(n=290)$ of the 1,118 short seat-time students had at least four course attempts, while only $15.5 \%(n=78)$ of the long seat-time students met that course-attempt criterion.

While the above relative-frequency comparisons of students' numbers of course attempts during the two-year longitudinal period sheds some light on students' mathematics-course persistence rates, it fails to provide information about a more important matter: course success. It is important, for instance, to keep in mind that some students who had numerous course attempts during the two-year tracking period had such high numbers due, either partly or entirely, to the fact that they were repeatedly unsuccessful in a given course and, hence, re-enrolled in it multiple times.

In disentangling course attempts (i.e., persistence) from course successes, it makes sense to start with the mathematics course that immediately follows MAT0024 Elementary Algebra in the algebra course sequence. That course is MAT1033 Intermediate Algebra. By the end of the Summer-2003 semester (which marked the end of the study's two-year longitudinal tracking period), a total of $749(22.8 \%)$ of the total student cohort $(N=3,284)$ had earned credit for MAT1033. It is important to note that this statistic does not include students who were successful during the tracking period in an equivalent MAT1033 class at another institution.

Students who passed MAT1033 during the tracking period were more likely to do so immediately after their Fall-2001 MAT0024 enrollment than during any other semester within the tracking period thereafter. In fact, the number of cohort students who 
passed MAT1033 each semester formed a monotonically decreasing sequence; that is, $394(52.6 \%)$ of the 749 students who earned a passing grade in MAT1033 did so during the Winter-2002 semester. After that, 240 (32.0\%) did so in Summer-2002, followed by $100(13.4 \%)$ in Fall-2002, 14 (1.9\%) in Winter-2003, and only one (0.1\%) in Summer2003.

As previously mentioned, a passing grade in MAT1033 is the course prerequisite for entrance into the first four general-education mathematics courses (GEMCs). Table 26 summarizes each student's total number of successfully completed GEMCs at the given institution and within the six-semester longitudinal tracking period.

Table 26

Disaggregated Frequency Distribution of GEMC Course Successes

\begin{tabular}{|c|c|c|c|c|c|c|c|c|}
\hline \multirow{3}{*}{$\begin{array}{c}\text { \# of GEMCs } \\
\text { Passed w/in } \\
\text { Tracking Period }\end{array}$} & \multicolumn{6}{|c|}{ Seat Time } & & \\
\hline & \multicolumn{2}{|c|}{ Short } & \multicolumn{2}{|c|}{ Medium } & \multicolumn{2}{|c|}{ Long } & \multicolumn{2}{|c|}{ Total } \\
\hline & $n$ & $\%$ & $n$ & $\%$ & $n$ & $\%$ & $n$ & $\%$ \\
\hline 0 & 959 & $(85.8)$ & 1,458 & $(87.7)$ & 444 & $(88.1)$ & 2,861 & $(87.1)$ \\
\hline 1 & 83 & $(7.4)$ & 118 & (7.1) & 34 & $(6.7)$ & 235 & $(7.2)$ \\
\hline 2 & 59 & (5.3) & 69 & $(4.2)$ & 17 & (3.4) & 145 & $(4.4)$ \\
\hline 3 & 16 & (1.4) & 15 & $(0.9)$ & 7 & (1.4) & 38 & $(1.2)$ \\
\hline 4 & 1 & $(0.1)$ & 2 & $(0.1)$ & 2 & $(0.4)$ & 5 & $(0.2)$ \\
\hline Total & 1,118 & $(100.0)$ & 1,662 & $(100.0)$ & 504 & $(100.0)$ & 3,284 & $(100.0)$ \\
\hline
\end{tabular}

As the table shows, $423(12.9 \%)$ of the 3,284 cohort student passed one or more GEMCs during the study's tracking period. When disaggregated according to the student's Fall2001 MAT0024 seat-time level, it was found that 159 (14.2\%) of the 1,118 short seattime students successfully completed at least one GEMC, whereas only $12.3 \%(n=204)$ and $11.9 \%(n=60)$ of the medium and long seat-time students, respectively, passed at least one GEMC. 
A two-way contingency table (not shown here) was then created to compare the trichotomous seat-time level variable against the dichotomous variable of whether a student was successful in at least one GEMC (coded as $1=$ Yes and $0=$ No). Then, a chisquare test for homogeneity of proportions was performed on this six-cell table, from which it was concluded that there was insufficient evidence $\left(\chi^{2}=2.819, d f=2, p=.244\right)$ upon which to conclude that a statistically significant difference existed.

It should be noted that the State of Florida, like many states, has for many years required a minimum of six credits (i.e., two 3-credit courses) of general-education mathematics coursework for awardance of the Associate in Arts degree and/or transfer admission into a baccalaureate-level program. Therefore, the reader may find it informative to focus on student completion rates for two or more GEMCs, instead of just one or more.

\section{Construction of DTSA Life Table}

In terms of the survival analysis's time metric (as previously defined), the smallest survival-event (or hazard-occurrence) time was 1.5 semesters. That is to say, the middle of the 12-week Summer-2002 semester was the earliest that any Fall-2001 MAT0024 cohort students successfully completed their first GEMC. Sixty-nine of the 423 survival events transpired at that time. Table 27 provides, in a full-cohort aggregated manner, the full chronology of GEMC survival-event times. 
Table 27

Survival-Event Frequencies by Consecutive Time Period

\begin{tabular}{|c|c|c|c|c|}
\hline $\begin{array}{l}\text { Time } \\
\text { Elapsed } \\
\text { (in sem.) }\end{array}$ & $\begin{array}{c}\text { Academic Semester } \\
\text { Equivalent }\end{array}$ & $f$ & $\begin{array}{c}\% \text { of total } \\
\text { cohort } \\
(N=3,284)\end{array}$ & $\begin{array}{c}\% \text { of total } \\
\text { survival events } \\
\quad(n=423)\end{array}$ \\
\hline 1.5 & middle of Summer-2002 & 69 & 2.1 & 16.3 \\
\hline 2.0 & end of Summer-2002 & 40 & 1.2 & 9.5 \\
\hline 3.0 & end of Fall-2002 & 135 & 4.1 & 31.9 \\
\hline 4.0 & end of Winter-2003 & 105 & 3.2 & 24.8 \\
\hline 4.5 & middle of Summer-2003 & 40 & 1.2 & 9.5 \\
\hline 5.0 & end of Summer-2003 & 34 & 1.0 & 8.0 \\
\hline & Totals & 423 & 12.9 & 100 \\
\hline
\end{tabular}

Tables 28, 29, and 30 below provide the respective actual-method life tables for each seat-time level. Only the columns of primary importance are shown, although others (e.g., the probability density, the hazard rate, and their respective standard errors) contributed to the computation of the estimated survivor functions. For reasons explained previously (in Chapter 3), the semester time-metric was partitioned into half-semester subinterval widths. 
Table 28

\begin{tabular}{|c|c|c|c|c|c|c|c|}
\hline \multicolumn{8}{|c|}{ Life Table for Research Question 3-Short Seat Time } \\
\hline \multirow[b]{2}{*}{$\begin{array}{c}\text { Interval } \\
\text { Start } \\
\text { Time } \\
\text { (\# of sem } \\
\text { after } \\
\text { Fall-'01) } \\
\end{array}$} & \multirow[b]{2}{*}{$\begin{array}{l}\text { At-Risk } \\
\text { Entering } \\
\text { interval } \\
\end{array}$} & \multicolumn{3}{|c|}{ Number of Students } & \multicolumn{3}{|c|}{ Proportion of Students } \\
\hline & & $\begin{array}{c}\text { Censored } \\
\text { during } \\
\text { interval } \\
\end{array}$ & $\begin{array}{c}\text { Exposed } \\
\text { to } \\
\text { GEMC } \\
\text { Risk } \\
\end{array}$ & $\begin{array}{c}\text { Had } \\
\text { (GEMC) } \\
\text { Hazard } \\
\text { Event } \\
\text { Occurrence }\end{array}$ & $\begin{array}{c}\text { Had } \\
\text { GEMC } \\
\text { within time } \\
\text { interval } \\
\text { [Hazard } \\
\text { Function] } \\
\end{array}$ & $\begin{array}{l}\text { Surviving at } \\
\text { End of } \\
\text { Interval } \\
\text { (among at- } \\
\text { risk at start of } \\
\text { time interval) }\end{array}$ & $\begin{array}{l}\text { Surviving at } \\
\text { End of } \\
\text { Interval } \\
\text { (cumulative) } \\
\text { [Survivor } \\
\text { Function] } \\
\end{array}$ \\
\hline .0 & 1118 & 0 & 1118 & 0 & .00 & 1.00 & 1.00 \\
\hline .5 & 1118 & 0 & 1118 & 0 & .00 & 1.00 & 1.00 \\
\hline 1.0 & 1118 & 0 & 1118 & 0 & .00 & 1.00 & 1.00 \\
\hline 1.5 & 1118 & 0 & 1118 & 32 & .03 & .97 & .97 \\
\hline 2.0 & 1086 & 0 & 1086 & 13 & .01 & .99 & .96 \\
\hline 2.5 & 1073 & 0 & 1073 & 0 & .00 & 1.00 & .96 \\
\hline 3.0 & 1073 & 0 & 1073 & 47 & .04 & .96 & .92 \\
\hline 3.5 & 1026 & 0 & 1026 & 0 & .00 & 1.00 & .92 \\
\hline 4.0 & 1026 & 0 & 1026 & 38 & .04 & .96 & .88 \\
\hline 4.5 & 988 & 0 & 988 & 15 & .02 & .98 & .87 \\
\hline 5.0 & 973 & 959 & 493.5 & 14 & .03 & .97 & .85 \\
\hline
\end{tabular}


Table 29

\begin{tabular}{|c|c|c|c|c|c|c|c|}
\hline \multicolumn{8}{|c|}{ Life Table for Research Question 3 - Medium Seat Time } \\
\hline \multirow[b]{2}{*}{$\begin{array}{c}\text { Interval } \\
\text { Start } \\
\text { Time } \\
\text { (\# of sem } \\
\text { after } \\
\text { Fall-'01) } \\
\end{array}$} & \multirow[b]{2}{*}{$\begin{array}{c}\text { At-Risk } \\
\text { Entering } \\
\text { interval }\end{array}$} & \multicolumn{3}{|c|}{ Number of Students } & \multicolumn{3}{|c|}{ Proportion of Students } \\
\hline & & $\begin{array}{c}\text { Censored } \\
\text { during } \\
\text { interval } \\
\end{array}$ & $\begin{array}{c}\text { Exposed } \\
\text { to } \\
\text { GEMC } \\
\text { Risk } \\
\end{array}$ & $\begin{array}{c}\mathrm{Had} \\
\text { (GEMC) } \\
\text { Hazard } \\
\text { Event } \\
\text { Occurrence }\end{array}$ & $\begin{array}{c}\text { Had } \\
\text { GEMC } \\
\text { within time } \\
\text { interval } \\
\text { [Hazard } \\
\text { Function] }\end{array}$ & $\begin{array}{l}\text { Surviving at } \\
\text { End of } \\
\text { Interval } \\
\text { (among at- } \\
\text { risk at start of } \\
\text { time interval) }\end{array}$ & $\begin{array}{l}\text { Surviving at } \\
\text { End of } \\
\text { Interval } \\
\text { (cumulative) } \\
\text { [Survivor } \\
\text { Function] }\end{array}$ \\
\hline .0 & 1662 & 0 & 1662 & 0 & .00 & 1.00 & 1.00 \\
\hline .5 & 1662 & 0 & 1662 & 0 & .00 & 1.00 & 1.00 \\
\hline 1.0 & 1662 & 0 & 1662 & 0 & .00 & 1.00 & 1.00 \\
\hline 1.5 & 1662 & 0 & 1662 & 25 & .02 & .98 & .98 \\
\hline 2.0 & 1637 & 0 & 1637 & 17 & .01 & .99 & .97 \\
\hline 2.5 & 1620 & 0 & 1620 & 0 & .00 & 1.00 & .97 \\
\hline 3.0 & 1620 & 0 & 1620 & 67 & .04 & .96 & .93 \\
\hline 3.5 & 1553 & 0 & 1553 & 0 & .00 & 1.00 & .93 \\
\hline 4.0 & 1553 & 0 & 1553 & 58 & .04 & .96 & .90 \\
\hline 4.5 & 1495 & 0 & 1495 & 20 & .01 & .99 & .89 \\
\hline 5.0 & 1475 & 1458 & 746 & 17 & .02 & .98 & .87 \\
\hline
\end{tabular}


Table 30

\begin{tabular}{|c|c|c|c|c|c|c|c|}
\hline \multicolumn{8}{|c|}{ Life Table for Research Question 3 - Long Seat Time } \\
\hline \multirow{7}{*}{$\begin{array}{c}\text { Interval } \\
\text { Start } \\
\text { Time } \\
\text { (\# of sem } \\
\text { after } \\
\text { Fall-'01) } \\
\end{array}$} & \multicolumn{4}{|c|}{ Number of Students } & \multicolumn{3}{|c|}{ Proportion of Students } \\
\hline & \multirow{6}{*}{$\begin{array}{l}\text { At-Risk } \\
\text { Entering } \\
\text { interval }\end{array}$} & \multirow{6}{*}{$\begin{array}{c}\text { Censored } \\
\text { during } \\
\text { interval }\end{array}$} & \multirow{6}{*}{$\begin{array}{c}\text { Exposed } \\
\text { to } \\
\text { GEMC } \\
\text { Risk } \\
\end{array}$} & \multirow{6}{*}{$\begin{array}{c}\text { Had } \\
\text { (GEMC) } \\
\text { Hazard } \\
\text { Event } \\
\text { Occurrence } \\
\end{array}$} & \multirow{6}{*}{$\begin{array}{c}\text { Had } \\
\text { GEMC } \\
\text { within time } \\
\text { interval } \\
\text { [Hazard } \\
\text { Function] }\end{array}$} & \multirow{6}{*}{$\begin{array}{l}\text { Surviving at } \\
\text { End of } \\
\text { Interval } \\
\text { (among at- } \\
\text { risk at start of } \\
\text { time interval) }\end{array}$} & \multirow{6}{*}{$\begin{array}{c}\text { Surviving at } \\
\text { End of } \\
\text { Interval } \\
\text { (cumulative) } \\
\text { [Survivor } \\
\text { Function] }\end{array}$} \\
\hline & & & & & & & \\
\hline & & & & & & & \\
\hline & & & & & & & \\
\hline & & & & & & & \\
\hline & & & & & & & \\
\hline .0 & 504 & 0 & 504 & 0 & .00 & 1.00 & 1.00 \\
\hline .5 & 504 & 0 & 504 & 0 & .00 & 1.00 & 1.00 \\
\hline 1.0 & 504 & 0 & 504 & 0 & .00 & 1.00 & 1.00 \\
\hline 1.5 & 504 & 0 & 504 & 12 & .02 & .98 & .98 \\
\hline 2.0 & 492 & 0 & 492 & 10 & .02 & .98 & .96 \\
\hline 2.5 & 482 & 0 & 482 & 0 & .00 & 1.00 & .96 \\
\hline 3.0 & 482 & 0 & 482 & 21 & .04 & .96 & .91 \\
\hline 3.5 & 461 & 0 & 461 & 0 & .00 & 1.00 & .91 \\
\hline 4.0 & 461 & 0 & 461 & 9 & .02 & .98 & .90 \\
\hline 4.5 & 452 & 0 & 452 & 5 & .01 & .99 & .89 \\
\hline 5.0 & 447 & 444 & 225 & 3 & .01 & .99 & .88 \\
\hline
\end{tabular}

An interval-to-interval comparison of each seat-time level's estimated survivor function reveals that, during no subinterval, did the proportion of students yet to experience their first GEMC success differ by more than .03 (i.e., $3 \%$ ); in fact, a difference of that magnitude did not occur until the end of the tracking period, when the event times for all student cases who had not yet succeeded in a GEMC were right censored.

Figure 4 displays the graphs of the estimated survivor functions shown in Tables 28-30. It displays graphically what the life tables did in tabular form; namely, the close proximity between — and repeated intersections among — the three seat-time levels' respective survivor-function values across the five-semester (Spring-2002 through Summer-2003) tracking period. 


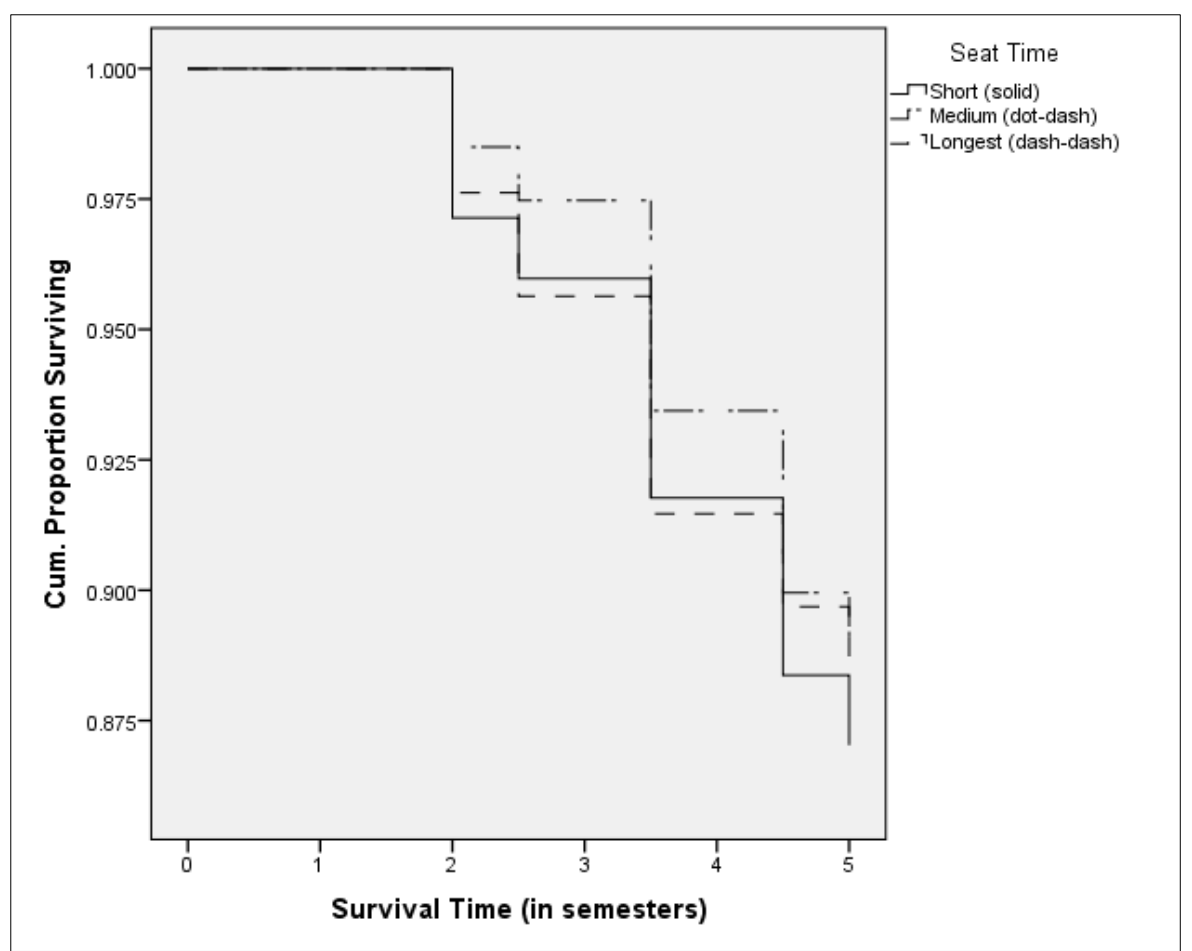

Figure 4. Graph of Q3's estimated survivor functions (half-semester partitioning).

It is important to remember that the survivor function, as its name indicates, describes the estimated proportion of subjects at each moment in time who had not yet experienced what survival-analysis methodology refers to as the hazard event. In this particular analysis, the survivor function provides, on a period-to-period basis, the proportion of students within a given seat-time level who had not yet successfully completed their first GEMC. Therefore, because non-survivors are those who actually had completed successfully at least one GEMC during the tracking period and they are the group of primary relevance to this research question, it is helpful to view (as shown in Figure 5) the estimated non-survivor function, which is also known as the cumulative-hazard function. 


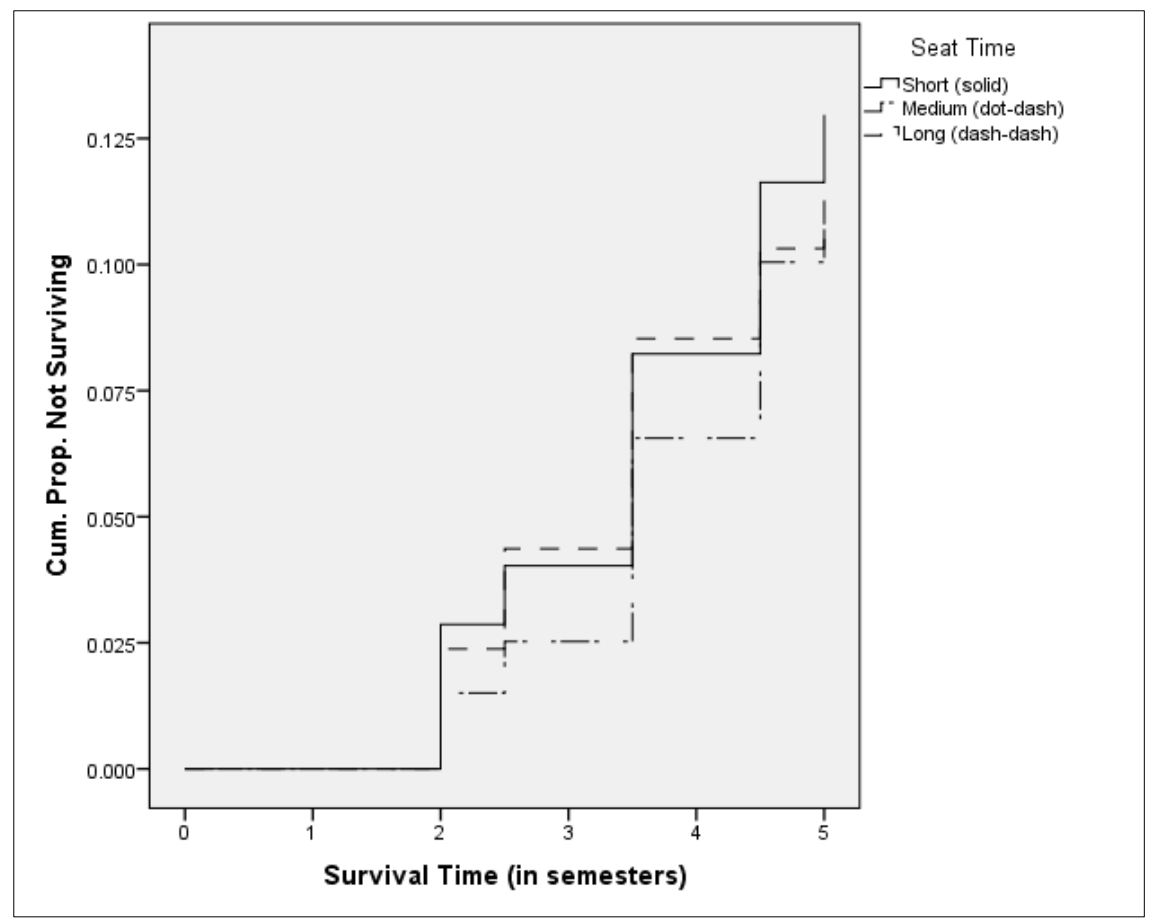

Figure 5. Graph of Q3's estimated non-survivor (cumulative-hazard) functions.

\section{Results of Life-Table Differences Hypothesis Test}

Based upon the similar trajectories among their respective life tables and survivor functions, it is reasonable to conjecture that there is no significant difference in the time to successfully complete a general-education mathematics course (GEMC) between students who completed their Fall-2001 MAT0024 course attempt in short versus medium versus long seat-time apportionments.

Nonetheless, a hypothesis test was performed; specifically, the actuarial method's Wilcoxon (Gehan) test. Two types of seat-time level differences were examined: overall and pairwise. The overall comparison yielded a Wilcoxon (Gehan) test-statistic value $\left(\chi^{2}\right.$ $=2.831, d f=2, p=.243$ ) that was not statistically significant. The pairwise analysis, as shown in Table 31, also failed to indicate any significant differences. 
Table 31

Q3 - Wilcoxon (Gehan) Test of Pairwise Life Table Comparisons ${ }^{a}$

(I)

(J) Wilcoxon (Gehan)

\begin{tabular}{ccccc} 
Seat Time & Seat Time & $\chi^{2}$ & $d f$ & Sig. \\
\hline \multirow{2}{*}{0} & 1 & 2.522 & 1 & .112 \\
& 2 & 1.302 & 1 & .254 \\
\hline \multirow{2}{*}{1} & 0 & 2.522 & 1 & .112 \\
& 2 & .001 & 1 & .976 \\
\hline \multirow{2}{*}{2} & 0 & 1.302 & 1 & .254 \\
& 1 & .001 & 1 & .976 \\
\hline
\end{tabular}

${ }^{\mathrm{a}}$ Comparisons are exact.

Finally, it should be noted that no Kaplan-Meier (KM) method confirmatory analysis was conducted because, unlike this study's second research question, this third research question's time metric was so heavily discretized. That is to say, only 11 survival-time values were possible, and actual hazard-event occurrences transpired at only six of these time values. Consequently, because ties in hazard-event times were so rampant and inevitable, the KM method's underlying distribution assumptions were not at all satisfied (Hosmer et al., 2008; Hox, 2010; Singer \& Willett, 2003).

\section{Summary}

This chapter provided the results of the quantitative analyses performed, while simultaneously addressing technical specifics pertaining to the hierarchical logisticregression model-building process and the survival-analysis techniques. Prior to stating the findings pertaining to each of the study's three research questions, descriptive analyses of the sample's demographic and academic-related covariates were presented.

Discussion of the findings, including their implications and limitations, and recommendations for future research and practice are presented in Chapter 5. 


\section{CHAPTER V}

\section{SUMMARY, CONCLUSIONS, \& RECOMMENDATIONS}

The objectives of this conclusory chapter are to summarize the study and the results of the analyses, to elaborate upon their implications, and to provide recommendations that hopefully will benefit other practitioners, policymakers, and researchers.

\section{Summary of Study}

While conducting this study, the researcher was driven by two supreme motives. The first was to use the seat-time-apportionment facet of course scheduling as a vehicle by which to persuade institutional leaders, academic- and student-services administrators, and instructional faculty to re-assess their course-scheduling decision-making processes and, in so doing, to give greater weight/attention to the myriad of effects such decisions may have upon student learning, course success, enrollment persistence, and time to program completion - especially in skill-based, prerequisite-driven disciplines like mathematics. Advances in neuroscience, along with relevant cognitive-learning and social-development theories, ought to play a pivotal, guiding role whenever formulating an institution's course schedule. "Given that the intended outcome of such decisions is to promote durable learning, understanding how the scheduling of study influences memory retention is critically important" (Carpenter, Cepeda, Rohrer, Kang, \& Pashler, 2012). At all times, it is important to bear in mind a nuanced awareness of inter-disciplinary and institutional-context differences (Chen, 2012; Murnane \& Willett, 2011; Titus, 2004).

The researcher's second paramount aspiration was to demonstrate to educational researchers, practitioners, and, especially, institutional-research/business-intelligence 
professionals that the types of multilevel, longitudinal analyses which have been embraced over the past two decades by large segments of the research community within other social-science disciplines are accessible, practicable, yet still greatly under-utilized in the field of educational research (Romero et al., 2005; Singer \& Willett, 2004).

America's public community colleges pride themselves on their societal role as open-door institutions dedicated to providing postsecondary educational opportunities to a highly eclectic population of adult, often non-traditional, students. As such, many community college leaders are fervently dedicated to expanding the range and accessibility of the services their institution's provide, in a perpetual effort to meet the ever-evolving educational and vocational-training needs of their respective communities. A large component of these expansion efforts entails proliferating new course-design formats; in particular, seat-time options that are intended to better accommodate the scheduling preferences of the nontraditional student.

For some community college leaders, their aim of "let us strive to attract and serve every prospective student we possibly can" is predicated largely, if not entirely, upon an activist-minded zeal for social justice, a motivation that many likely would characterize as pure and commendable. In contrast, the driving ambition of other leaders is to increase institutional market share in what has become over the past two decades a hyper-competitive "knowledge provider industry." Increasingly nebulous, this industry is comprised of a wide array of entities - ranging from proprietary institutions to corporatetraining providers to online programs, among others—-fiercely vying for "student clientele.” 
In both cases unfortunately, no matter whether the predominating motives of a community college's leadership when expanding their institution's offerings of coursedelivery and seat-time scheduling options are more altruistic or more capitalistic, two critically important, detrimental side effects are often overlooked.

First, as this dissertation's introductory chapter and the multi-faceted theoretical framework undergirding it showed, cognitive and developmental theory and related advances in neuroscience play, at best, a marginal role in many institutions' course scheduling processes. For at-risk 2-year college students enrolled in developmental-level courses, this decision-making deficiency can have especially severe consequences.

For example, as advances in the digital information age continue to spawn a rapidly expanding wealth of beyond-the-classroom learning resources, some have posited that course scheduling considerations like seat-time apportionment are less relevant and increasingly anachronistic. Their rationale is essentially this: even if one were to concede that decades of robust research findings have demonstrated that lengthier, massed class sessions decrease the quality and quantity of a learner's conceptual comprehension, content retention, and skill mastery in a variety of cognitive domains (Kornell \& Bjork, 2008), today's students are different because they have ubiquitous access to asynchronous multimedia tools that should fill in any learning gaps and more than amply compensate for less-than-ideal course seat time durations. It turns out, however, that there are various flaws in the supposition that today's adult learners will be sufficiently adept at self-managing and self-initiating their learning episodes, a notion that in the literature is often termed self-regulated learning. One thorough review of the recent research on selfregulated learning (Bjork, Dunlosky, \& Kornell, 2013) revealed that “...people often 
have a faulty mental model of how they learn and remember, making them prone to misassessing and mismanaging their own learning” (p. 417). Another recent metaanalysis of more than 430 independent samples from 369 studies revealed that, among the 16 self-regulatory processes analyzed, the two strongest predictors of successful selfregulated learning are goal-setting and self-efficacy (Sitzmann \& Ely, 2011). A large majority of remedial-level community college students, however, are deficient in such behaviors (Deil-Amen \& Rosenbaum, 2002; Rosenbaum, 2011), which further lends credence to the contention that, in this multimedia information age, the spacing effectand, in particular, seat-time apportionment—still remain important considerations for 2year college students.

Second, there is a flawed tendency to conflate the decision-making capacity and academic maturity of enrollees at open-door community colleges with that of freshmanand sophomore-level undergraduates admitted to 4-year institutions. As Rosenbaum et al. (2007) concluded from their extensive multivariate research, “... most students at twoyear colleges would not be in college if two-year colleges did not exist" (p. 50). As evidenced by the high proportion whose low placement test scores warrant developmental coursework, many community college students have meager academic histories.

Although community college educators are keenly aware of their students' academic prerequisite deficits (in basic fundamentals like reading, writing, and arithmetic) because their institutions dedicate a considerable proportion of resources and personnel to help students remediate these deficiencies, it is disconcerting that what would seem to be the three most obvious intrapersonal outgrowths of these educational- 
background shortcomings are seldom considered when configuring course schedules and, in turn, attempting to niche market a complex variety of seat-time/delivery-system options: (a) 2-year college students' often deficient information-processing and decisionmaking skills, particularly with regard to educational and career-related matters; (b) confusion and unfamiliarity with the demands, the vernacular, and the expectations of college; and (c) low levels of intellectual self-esteeem and self-confidence that manifest themselves in student tendencies such as trying to "course shop" for the perceived path of least resistance and then despairing when they realize their perceptions were mistaken.

Such students tend to be ill equipped to determine which course option is most suitable for maximizing their learning gains. For many of them, it would be an alien notion to even begin to think in those terms, for learning quality is, at best, a low priority. Even traditional-age (18-22-year-old) full-time 2-year college students are inclined to select their course schedules based upon non-academic criteria; for example, identifying course sections that will require the fewest number of trips to a campus and that can be squeezed around one's part-time work schedule.

Paradoxically, despite the incommensurability of their value system and insufficiency in social capital, many of these very same students continue to have lofty, unrealistic scholastic aspirations. As Rosenbaum (2011) characterized it, Even more pernicious, a [societal college-for-all and higher education system student-recruitment-at-all-costs] focus on encouraging plans for bachelor's and master's degrees among low-achieving [high school] seniors.... encourages unrealistic dreams and prevents students from considering realistic backup 
options or programs that give short-term certificates and associate's degrees on the way to their goals for bachelor's degrees. (p. 116).

For these reasons, too many course scheduling options, however well intentioned, inadvertently create maze-like, dead-end pathways for students that exacerbate the hazardous effects of their information-processing, decision-making, and self-confidence deficits. For students in mega-states like Florida and Texas that have mandated limitations on (and financial penalties for) repeating a college course, the consequences can be especially catastrophic. As Rosenbaum et al. (2007) explain, Many two-year college students face more challenges than the average college student, and motivation and confidence are even more important at these schools, given many students' poor academic history and lack of college exposure. These students often need more certainty than many community colleges offer. (p. 53) The nonprofit organization Complete College America (2012) has synthesized the research of Rosenbaum and a number of other prominent scholars into an 104-page solutions-oriented report. It recommends, for example, that institutions "close remediation exit ramps... create clear, limited, and structured program pathways containing core college-level courses. Then require students to choose a pathway" (p.12). Furthermore, the report's authors argue:

Students should make the big choices of programs of study informed with an understanding of program requirements and available supports to achieve their career goals. Once they do, place them into structured program pathways constructed of relevant, sequenced courses chosen for them. (p.11) 


\section{Summary of Results}

This study entailed the collection and analysis of a retrospective, longitudinal quantitative data set in an effort to respond to the following three research questions:

Research Question 1: In community-college developmental-level algebra courses, is there a difference in student success for students enrolled in classes that meet one day per week in longer-duration class meetings versus for those enrolled in mediumduration (twice weekly) class meetings versus for those enrolled in shorterduration (thrice weekly) class meetings?

Research Question 2: In community-college developmental-level algebra courses, is discrete survival time, as measured by date of withdrawal from the course, related to the number of class meetings per week and the duration of each meeting?

Research Question 3: Across the sequence of community-college algebra courses, is there a longitudinal difference in the time to complete at least one general-education college-level mathematics course between students who satisfied the prerequisite algebra course requirements by completing one or more algebra courses scheduled in a one-day-per-week, longer-duration format and students whose prerequisite algebra course requirements were entirely fulfilled in courses that had medium-duration (twice weekly) or shorter-duration (thrice weekly) class meetings?

For the first research question, hierarchical linear modeling analyses (namely, two-level logistic regression) were conducted on a multivariable data set comprised of a particular student cohort $(N=3,284)$ who were enrolled in the 120 Fall-2001 class sections of MAT0024 Elementary Algebra offered at a large multi-campus community college 
located in urban South Florida. In excess of 50 covariates were considered in the multivariable model-testing process. The vast majority of these covariates were selected based upon the theory-driven recommendations of other researchers (e.g., Andreu, 2002; Bini et al., 2009; Fike \& Fike, 2008; Goble, Rosenbaum, \& Stephan, 2008; Moosai et al., 2011). These included student-demographic, instructor-demographic, academicbackground, and class-section related variables. Additional variable-selection insights were garnered from journal articles in which the author(s) reviewed the existing literature on 2-year college student success variables (e.g., Burns, 2010)

Although the analyses failed to demonstrate a direct, statistically significant predictive relationship between the class section's seat-time level and the student's course success, a significant cross-level interaction effect between seat-time level and enrollment-status type (i.e., full-time versus part-time) was found. However, this interaction effect was limited in that statistical significance $(\mathrm{p}<.01)$ was detected at only one interaction level: a full-time student enrolled in a short seat-time class section.

Follow-up cross tabulations revealed that full-time student success rates were highest in short seat-time classes (49.1\%) and lowest in long seat-time classes (38.8\%), while $44.2 \%$ of full-time students in medium seat-time classes were successful. The reverse was true for part-time students, for whom $46.5 \%$ of long seat-time enrollees were successful, whereas only $40.5 \%$ and $36.3 \%$ of their medium and short seat-time counterparts were successful, respectively. It is reasonable to speculate that one reason why the part-time students tended to be less successful in short seat-time class sections was the increased time availability required to travel to campus three or more times per week, which often poses a hardship for part-time students, many of whom are employed 
on a full-time basis and are more likely than full-time students to have adult familial responsibilities.

With regard to why full-time students enjoyed lower likelihoods of success in long seat-time sections, the findings of Nelson Laird and Cruce (2009) may be instructive. They found that full-time students are negatively affected by institutional cultures predominated by part-time students. This indeed was the classroom culture in long seat-time MAT0024 sections, which were comprised of a nearly 5:1 ratio of parttime to full-time enrollees. Additionally, the spacing effect's implications may have been more consequential for full-time students rather than part-time, due to the quantity and range of information full-time students must process. This would be consistent with the theory of deficient processing (Toppino \& Bloom, 2002), which supports the notion that appropriately spaced learning episodes enhances the quantity and quality of the information processed.

In addition to this seat-time/enrollment-status cross-level interaction effect, the final parsimonious model constructed identified a set of nine statistically significant covariates (all with $p$-values less than .01) as significantly related to success in the MAT0024 developmental-mathematics course. These nine covariates were in three separate categories: student-demographic related Level-1 variables (sex, age, dichotomous coding of Black/African-American racial classification, and immigration status); course-section related Level-2 variables (instructor age); and student academichistory related Level-1 variables (total number of semester hours attempted and the total number of semester hours earned both within the institution and at other postsecondary institutions prior to enrollment in the MAT0024 course, the total number of previous 
attempts of the other two developmental-level courses at the institution, and whether a student learning/life skills course had been previously attempted).

Among these nine statistically significant covariates, there were four that were especially notable in terms of the magnitude of their odds ratios in the final two-level logistic regression model.

The first was the total number of MAT0012 Pre-Algebra and/or MAT0020 Combined Pre-Algebra/Elementary Algebra course attempts each student had at the institution prior to the Fall-2001 semester. To enhance the interpretability of its exponentiated coefficient, this ratio-level variable was grand-mean centered. As an academic-history variable, it stood apart from the remaining three notable covariates, all of which were nominal-level student-demographic attributes. The final fitted model revealed that, when all other covariate values are fixed, the likelihood of a student's success in MAT0024 decreases by approximately 35\% for each additional previous attempt at MAT0012/0020.

Some might submit that this finding is contrary to Bjork and Bjork's (1992) new theory of disuse, which contends that spacing the distribution of practice induces greater memory storage strength than does bunching of repetitions. Hence, some may view repeated MAT0012/0020 course experiences as a form of prolonged distributed practice. However, in the case of this particular covariate, there are other superseding factors that may better explain its inverse relationship with MAT0024 course success. For example, a high number of MAT0012/0020 course attempts is likely to be indicative of a student having a greater severity of mathematical deficiencies, both in terms of skill development and conceptual comprehension. Often, such students have a higher rate of remedial-level 
placement in low-level reading and writing coursework, which is yet another obstacle to success in courses such as MAT0024 Elementary Algebra. Additionally, social psychological theories, such as the identity development theory known as the stereotype threat, may shed greater light on the finding of an inverse relationship between MAT0012/0020 course attempts and MAT0024 course success. The stereotype threat refers to the notion that the weight of stereotypes about particular groups can impair an individual's performance, especially in high-stakes, evaluative situations (Aronson, 2004; Syed, Azmitia, \& Cooper, 2011). Certainly, the demands of a postsecondary mathematics course fall into that category of situation, and so it is conceivable that repeated remedial course attempts may inculcate negative self-perceptions that diminish a student's intellectual self-esteem level and, thus, adversely affect performance.

The second of four covariates that displayed a large odds ratio pertained to a student's sex. When holding all other covariates constant, a female student's success odds in the MAT0024 Elementary Algebra course were found to be approximately $38 \%$ higher than a male student's success odds. This difference is smaller but consistent with the findings of Bailey, Jeong, and Cho (2010). Based upon their logistic regression analyses of 2003-2004 first-time registrants in 57 community colleges, they estimated the success odds of female developmental-level mathematics students to be 1.53-1.56 times as much as the odds for males (p. 264).

In this dissertation study, the aggregated passing rate was $45.4 \%(n=945)$ for the 2,080 female students but only $39.1 \%(n=469)$ for the 1,200 males. The male students' MAT0024 course success rates exhibited greater variability across the three seat-time levels: $40.3 \%(n=177)$ of the 439 men in short seat-time class sections, $37.2 \%(n=227)$ 
of the 610 men in medium seat-time classes, and $43.0 \%(n=65)$ of the 151 men who enrolled in long seat-time class sections. In contrast, the success rates of female students were quite consistent across the three seat-time levels: $46.2 \%(n=313)$ of the 678 women who enrolled in short seat-time class sections, $44.8 \%(n=470)$ of the 1,050 women in medium seat-time sections, and $46.0 \%(n=162)$ of the 352 women in long seat-time classes.

The third of the four covariates that exhibited a noteworthy odds ratio was race; in particular, whether a student's self-identified race was Black or not. Based upon his extensive review of postsecondary retention research, Reason $(2003 / 2009)$ concluded, "New studies must reexamine our understanding of [race, gender, ethnicity, age, and other demographic variables] and their relationships to retention. Sophisticated studies must examine the interaction of these variables to fully understand the differential experiences of various populations" (p. 187/p. 497). The social development theory known as the stereotype threat would be one among many examples of the "differential experiences" Reason (2003/2009) had in mind. Upon that rationale, this racial demographic covariate finding warrants mention, even despite its internal reliability shortcomings, as was discussed at length in the previous chapter and is further elaborated upon later in this closing chapter. A Black student's success odds in the MAT0024 Elementary Algebra course were found to be approximately 36\% lower than that of a non-Black student, when keeping all other covariates constant. Among students who selfidentified their race as Black $(n=1,119)$, the MAT0024 course success rate was $37.9 \%$ $(n=424)$, as compared to $45.8 \%$ of the 2,165 students who did not identify themselves as 
Black. Only $32.4 \%$ of the 343 self-identified Black males passed the course, whereas $40.3 \%$ of the 776 Black female students did so.

These findings - despite this researcher's concerns about the internal consistency of this demographic variable - are consistent with nearly all of the literature (Bailey et al., 2010; Cuyjet, 2006; Kuh et al., 2008; Perrakis, 2008; Reason 2003/2009). For instance, in their extensive research, Bailey et al. (2010) found, "Black students had particularly low [success] odds when they were referred to developmental math at two or three or more levels below college-level" (p. 264). Perrakis (2008) recommended that community college researchers and practitioners extend beyond the typical ways of analyzing demographic covariates and seek better ways to analyze (and act upon) lurking interaction effects:

Differences in levels of academic preparation may in fact supersede differences in race and gender; research and policy is needed to better understand and assist students at different levels of their academic careers. For now, emphasis remains on traditional measures of difference — race, class, gender—while these other categories of difference remain largely unexplored in the literature or institutional policy. (p. 22)

In the first research question's final logistic regression model, the last of the four covariates that exhibited a large magnitude in its odds ratio pertained to student immigration status. In the multi-level (GLMM) model building process, this polychotomous demographic variable was treated so that four distinct groupings of noncitizen classifications were individually compared against the reference category of U.S. citizen status. Among these four non-citizen categories, three were found to be 
statistically significant. First, when all other covariates were held constant, the MAT0024 Elementary Algebra success odds among F-1 Student Visa holders were found to be approximately 5 times greater than that of students who were U.S. citizens $(p<.001)$. Whereas the course success rate for the 2,557 U.S. citizens was $40.8 \%$, it was $78.1 \%$ for the 73 students who possessed an F-1 Visa. Second, the success odds for a student whose immigration status was in the Other Documented Type (ODT) category was found to be approximately 2.5 times greater than that of a student who was a U.S. citizen $(p<.001)$. This ODT category consisted of the researcher's consolidation of 14 different documentation statuses encompassing everything from asylees to tourists. Among the 81 ODT students in the Fall-2001 cohort, 64.2\% were successful in the MAT0024 course. Thirdly, the success odds for a student with permanent resident alien (PRA) status was estimated to be about 1.3 times larger than that of a student who was a U.S. citizen $(\mathrm{p}<$ .01). Of the 570 MAT0024 Fall-2001 cohort students who had PRA status, $46.0 \%$ earned a passing grade in the course.

Not only was this course success rate markedly lower than the rates for the ODT and F-1 Visa groups, it also exhibited a difference between the sexes. While the ODT and F-1 Visa success rates were higher for men than women (i.e., $67.6 \%$ to $61.7 \%$ for ODT, $79.2 \%$ to $77.6 \%$ for F-1 Visa), the results were quite different for PRA students: $34.9 \%$ of the 192 male students were successful, in contrast to a success rate of $51.6 \%$ for their 378 female counterparts.

In addition to between-sex differences, there was a marked difference between the tuition status (i.e., in-state resident versus out-of-state nonresident) of F-1 Visa students and that of other immigration statuses. More than $93 \%$ of (68 of 73) F-1 Visa students 
paid nonresident tuition, in contrast to a combined rate of only $8.6 \%$ (56 of 654) for the other three non-U.S. citizen immigration statuses (ODT, PRA, and undocumented). In the entire sample of 3,284 students, about $9.6 \%(n=314)$ were charged the out-of-state nonresident tuition rate, either because they were not Florida residents or because they were assessed a tuition penalty in accordance with Florida's three-attempt rule (as was discussed in Chapter 1). Among students who were U.S. citizens, the rate was 7.4\% (190 of 2,557). In light of the very high course success rate among F-1 Visa students (78.1\%) and the fact that the overall course success rate for students who paid nonresident-rate tuition (51.0\%) was higher than those who paid resident-rate tuition (42.3\%), tuition status may be a covariate, or perhaps an interaction effect, worthy of additional exploration.

For this study's second research question, discrete-time survival analysis (DTSA) methodology was employed. The analysis pertained to the same Fall-2001 developmental-mathematics course as the first research question did. However, they differed in one important respect: the first question examined group (seat-time) differences in course success, whereas the second question scrutinized seat-time group differences in student course-withdrawal times. The analysis revealed a statistically significant difference (at the $p<.05$ level) in student course-withdrawal time between the short (e.g., 50-minute three-day-per-week classes) and long (e.g., 150-minute one-dayper-week classes) seat-time levels. Specifically, students in long seat-time classes tended to withdraw at a faster and higher rate (i.e., earlier in the course) than did their peers in short seat-time classes. Neither the short nor long seat-time level's course-withdrawal times/rates, however, were found to be significantly different than that of the medium 
level (e.g., 75-minute two-day-per-week classes).

Additionally, it is tangentially noteworthy—in light of recent course-scheduling trends favoring condensed mini-term offerings - that the preliminary descriptive analyses performed for the second research question prior to the DTSA (as shown in Chapter 4's Table 18) revealed that the proportion of students who withdrew from the 20 mini-term (12-week) classes was more than $4 \%$ higher than that of the students from the 99 fullterm (16-week) classes (i.e., 21.6\% versus 17.3\%, respectively). A chi-square test for homogeneity of proportions demonstrated a statistically significant difference $(\mathrm{p}<.05)$. This finding may warrant further examination of the relationship between student persistence and course session length.

For the third research question, discrete-time survival analysis (DTSA) methodology was again utilized. In contrast to the second research question, though, the third question's DTSA pertained to a two-year (six-semester) longitudinal tracking period, not a one-semester course-based timespan. In the entire aggregated sample (i.e., without controlling for any demographic or other academic covariates), the analysis failed to reveal a significant difference in time (measured in elapsed semesters) to successfully complete a general-education mathematics course (GEMC) between students who completed their Fall-2001 MAT0024 course attempt in short versus medium versus long seat-time apportionments.

With regard to the findings pertaining to the third research question, it should be noted that, in the case of one demographic group (namely, Black/African-American students), a statistically significant longitudinal difference was detected $\left(\chi^{2}=10.188, d f=\right.$ $2, p=.006)$ but was purposefully omitted from Chapter 4. Pairwise follow-up analyses 
revealed that Black students were far less likely to complete at least one GEMC during the longitudinal tracking period if they had enrolled in a long seat-time MAT0024 Fall2001 class section when compared to those who had enrolled in a short-length $\left(\chi^{2}=\right.$

$5.010, d f=1, p=.025)$ or medium-length $\left(\chi^{2}=11.271, d f=1, p=.001\right)$ MAT0024

section. The main reason for ignoring this finding was, as discussed earlier in this dissertation, the low internal reliability and high nonresponse rates of the race/ethnicity covariates. This matter of concern is revisited later in this chapter.

\section{Some Unanticipated Longitudinal Limitations}

Two potential reasons for why the third research question's longitudinal DTSA failed to yield statistically significant results, in the aggregate, are especially noteworthy.

First, during the data-analysis process, the researcher discovered a lurking institutional-context variable of potentially significant import: at some Florida 2-year colleges, including the one from which this study's data were collected, students who had not yet fulfilled their developmental-level course requirements were not compelled to immediately re-enroll the next semester. Instead, during at least the first half of this study's 6-semester longitudinal tracking period (Fall-2001 through Summer-2003), they were virtually unhindered from self-registering in college-level coursework in other disciplines, in contrast to their peers who were attending a number of other Florida community colleges during this same time period.

That is to say, in the year 2000, Florida's State Board of Education revised its Rule 6A-10.0305 to require students who tested into one or more developmental-level courses to either successfully complete these requirements by the time they had accumulated 12 hours of college credit coursework or maintain continuous enrollment 
until they did so. The 2-year college that was the source of this study's data did not fully implement this rule into its student-registration, computer-system programming until at least the fall of 2002. This, therefore, allowed students who initially enrolled in, for example, a developmental-level algebra course in the Fall-2001 semester to have continued for several semesters to enroll in non-mathematics courses with little, if any, restriction. Meanwhile, in contrast, some other Florida 2-year colleges fully implemented the statewide rule more expeditiously. Hence, over the span of a 6-semester tracking period, their students' developmental-level enrollment patterns may have been markedly different than the institution examined in this study. So, in retrospect, a 6-semester span for GEMC successful completion may have been too short of a longitudinal period.

Secondly, during the research-design phase of this study, the researcher thought the sample size of the student cohort $(N=3,284)$ would be more than sufficient from a statistical-power perspective. In retrospect, however, this may not have been the case because: (a) $35.2 \%$ of the cohort $(n=1,157)$ did not enroll in any additional mathematics courses at the institution after their Fall-2001 MAT0024 course experience; (b) only 43.1\% $(n=1,416)$ of the cohort earned a passing grade in their Fall-2001 MAT0024 course, of which 327 did not enroll in any subsequent mathematics courses at the college during the remainder of the longitudinal period; and (c) the number of enrollees in long seat-time MAT0024 Fall-2001 classes $(n=504)$ was less than half of the number in the short-level $(n=1,118)$ sections and less than a third of those in the medium-level classes $(n=1,662)$

Furthermore, it is important to remember that MAT1033 Intermediate Algebra is an additional prerequisite course students must successfully complete prior to being 
eligible to enroll in a GEMC. MAT1033 has often been called a "bottleneck" course, which would suggest a further diminishment of the original cohort's sample size. These factors certainly warrant reconsideration of how large a sample size — and how lengthy a timespan - is needed for a longitudinal survival analysis with such a high student-attrition rate and an accompanying right-censored case rate.

The work of Moosai et al. (2011) provide some valuable insights into these timespan and sample-size issues. In their study of student and institutional predictors of community college graduation rates at 142 community colleges in three states, they attested to the erratic nature of community college students' enrollment patterns; most notably, part-time students, who encompass the majority of community college enrollment (p. 813). Moosai et al. (2011) compensated by restricting their sample to a three-year time period and a student cohort restricted to first-time, full-time, degreeseeking students. Although such a restriction on the student sample was not feasible in the case of this dissertation's single-institution study for a variety of reasons, it is nonetheless instructive that Moosai et al. (2011) utilized a three-year tracking period, even despite the comparatively massive size of their sample and, hence, the statistical power it afforded them.

\section{Some Very Practical Course-Scheduling Recommendations}

Far above and beyond the statistical results of this study, it is vital for practitioners and researchers alike to step back, reflect deeply upon, and periodically ponder the fundamental question upon which this study has endeavored to shed light: what course-scheduling configurations in terms of seat-time apportionment are in the best interest of developmental-level algebra students, both in the immediate (course-specific) 
term and across the longer-term span of their subsequent (and sequential) mathematics coursework? Although quantitative-analysis techniques like (multi-level) logistic regression and survival analysis can contribute to these types of institutional decisions, they are by no means to be misconstrued or misused as a surrogate for sound pedagogical, theory-driven judgment.

A well-constructed course schedule should take a wide variety of factors and constituencies into account. Although every academic discipline is different (e.g., a 3hour seat time may be best for an art major's hands-on ceramics course but would not be suitable for, say, a music major's one-on-one tuba lesson), what is in the best academicinterest of students in that discipline should be the paramount consideration. This, of course, should not be confused with over-catering to students' scheduling preferences, for many community-college commuter students would prefer to come to class as seldom as possible. Developmental-level students, in particular, are generally not equipped to know what pedagogical considerations will best serve their learning needs (Deil-Amen \& Rosenbaum, 2002; Rosenbaum et al,. 2007; Rosenbaum, 2011). Similarly, although instructor staffing is always an important consideration, full-time faculty work-hour preferences should be, at most, an ancillary decision-making factor when constructing a course schedule.

Having been exposed to the common concerns administrators, faculty, and students tend to voice at various public commuter-student colleges, this researcher wishes to make three main recommendations to those who oversee institution-wide enrollment management activities, as well as to those who are charged at the intra-disciplinary level with building a master course schedule; most notably, a 2-year college mathematics one. 
First, it is essential that institutions create initiatives devoted to upgrading enrollment-management protocols that are both perennial and mandated for all stakeholders responsible for the creation of the course schedule. Such activities need to be recurrent for three main reasons: to keep pace with rapid changes in asynchronous, multimedia learning technologies; to acquire a sufficient grounding in relevant cognitive psychology theory (e.g., the spacing effect) while staying apprised of advances in neuroscience and metacognition; and to equip course-scheduling decision makers with the tools, training, and support system necessary to be effective managers, especially in this era of frequently high turnover rates among college administrators. Mandating participation for all enrollment-management stakeholders - neophytes and veterans in course scheduling alike-is vitally important because: (a) a necessary condition of formulating a well-devised master course schedule (e.g., one that minimizes time-block scheduling conflicts for students in low volume courses) is cross-disciplinary interaction and a holistic-minded institutional ethos among all managers; (b) training sessions are to be regularly updated in such a way that new concepts and scheduling considerations are incorporated; (c) longtimers often have valuable tips and experiences to share with newcomers; and conversely, (d) newcomers' creativity and fresh ideas can help longtimers and, more generally, the institution avoid complacency and fatalism, which often contributes to derelict practices such as reflexively rolling over previous years' course schedules.

Secondly, course-scheduling decision-making practices need to pay greater attention to what is in students' longer-term, academic best interest. To do so, the metrics and methods used to gauge student success need to move beyond the superficiality of a 
purely cross-sectional (i.e., course-specific) perspective that is nothing more than aggregated course passing and persistence rates and, instead, move toward a more nuanced multi-level longitudinal view — especially in sequential skill-development and abstract-reasoning disciplines such as mathematics. For that evolution to occur, institutions must make a concerted effort to incorporate, in a way that is user-friendly to scheduling decision makers, these quantitative-analysis concepts into the training and initiatives described in the preceding paragraph. The first crucial step in that effort is to persuade executive-level leaders of the relevance, benefits, and practicability of these analytic devices.

Thirdly, based upon the rationale and research detailed earlier in this chapter, many 2-year colleges need to streamline their range of course-scheduling options so that pathways that are confusingly circuitous for (and ill-suited to the learning needs of) atrisk students are removed. Additionally, in concert with institutional research professionals, control processes need to be devised that will enable course-scheduling and course-delivery pilot initiatives to be methodically formulated and systematically evaluated, in an effort to improve future replication.

Perhaps, the best way to foster contemplation of and, subsequently, discourse among one's colleagues about the theory-driven and research-driven arguments put forward in defense of course-scheduling decision-making reform (especially with regard to 2-year college mathematics courses) is to express these suggested criteria in question form:

- Does a skill-driven, prerequisite-laden academic discipline like mathematics lend itself to learning episodes that are shorter and more frequent instead of larger and 
more massed? Said differently, is the process of becoming proficient in a skill, such as solving algebra problems, related to classroom seat-time duration?

- Does the many decades worth of robust research into learning theory's spacing effect deserve to play a more prominent role in course-scheduling decision-making processes? For example, in addition to the research cited in this dissertation's literature-review chapter, consider two other recent spacing-effect (distributedpractice) studies applicable to higher-education instruction in general and mathematics/statistics course scheduling in particular: (a) Budé, Imbos, van de Wiel, and Berger (2011) and (b) Carpenter et al. (2012).

- In light of how abstract traditional algebra/mathematics curricula are in comparison to that of many other academic subjects and in light of the symbolic manipulation and notational/terminology nuances inherent in algebra coursework, what seat-time apportionment level best accommodates developing students' conceptual understanding as they transition from (comparatively) concrete arithmetic to abstract algebraic notions?

- For developmental-level algebra students who generally have not had a history of past successes with mathematics and who often tend to be math phobic and/or math averse, do longer-length seat-time classes tend to compound their levels of math anxiety and dislike, especially when one considers that one-day-per-week, three-hour algebra (pure lecture-type) courses are lengthier in duration than most major motion pictures and other forms of entertainment, such as sporting events?

- In this era of rampant "multi-tasking" along with the rise in attention-deficit (ADHD) diagnoses over the past decade, are attention spans, on average, indeed getting 
shorter? If so, are longer-length, massed meetings even more problematic for community college students today (in 2012) than they were circa 2001?

- How much more severe are the consequences of a class-meeting cancellation—due to, for example, instructor illness, weather conditions (such as a snow storm or a tropical cyclone), or a power outage—-for a longer-length seat-time class section than a short/medium-length one? When a single one-day-per-week mathematics class meeting is cancelled, that amounts to a loss of approximately $1 / 15^{\text {th }}$ of the course. Is that not a significant loss of continuous instructional time in comparison to a single class cancellation for a two- or three-day-per-week class?

- Similarly, when students are absent from a single meeting of a one-day-per-week class, they miss one full continuous week of instruction. In contrast, two separate absences from a two-day-per-week class are often not consecutive. So, are isolated student absences from longer-duration seat-time mathematics classes more consequential in terms of (a) success in the present course and (b) exacerbating gaps in student background skills that may hinder future course success than an isolated absence from a shorter/medium-duration class?

- In advance of scheduling one-day-per-week classes, is care taken to check the calendar and the specific weekdays on which the holidays for that particular year will fall? With shorter/medium-length seat-time duration sections, the number of actual class meetings tends not to diverge significantly from, say, Monday-Wednesday versus Tuesday-Thursday classes. However, with one-day-per-week classes, it is not uncommon to see a particular day of the week affording students 16 class meetings (i.e., 48 hours of instructional contact time) versus another day affording only 13 
meeting dates (i.e., 39 hours of in-class instruction). How potentially problematic is this for students, both in terms of the quality of their course experience and their likelihoods of persistence? Also, how potentially problematic is this for instructors, in terms of consolidating the same amount of course content into 9 fewer hours of instruction while not compromising academic standards?

- For mathematics courses, do longer-length, less-frequent class meetings run the risk of undercutting (detracting from) the most frequently uttered study-skills message mathematics educators impart to their students; namely, a necessary condition for success in a skill-acquisition discipline like mathematics is frequent (if not daily) homework/practice sessions? Do one-day-per-week class meetings belie that advice and give postsecondary students (in particular, remedial-level students) the false impression that success in collegiate mathematics courses demands no more of a time commitment than course success in other non-skill-based academic subjects?

- Is the fact that one-day-per-week class offerings in 2-year college mathematics programs tend to be overwhelmingly, disproportionately scheduled for evening/weekend students an important concern? Does the institution run the risk of effectively running two colleges in one; that is, one set of standards and expectations for daytime students and another for night/weekend students? Where should the line separating accommodation from panderism be drawn? After all, is it not the case that, when left to their own devices, the vast majority of nontraditional and/or working students will prefer classes that require the fewest number of meeting dates?

- For those academic departments that in the past have casually experimented with but ultimately had to cancel courses scheduled during off-peak time blocks (e.g., mid- 
afternoons) and subsequently inferred that such scheduling options are not viable for their particular student population, were these off-peak sections scheduled improperly as a supplement to the pre-existing master schedule or, more prudently, as a substitute for course sections that in previous semesters were scheduled during peak-demand time blocks, such as mid-morning? If it were as an add-on, then is it not more likely that the off-peak course cancellations were attributable to the supply of course sections exceeding demand, instead of the specious inference that off-peak class sections will never garner sufficient enrollment? Since commuter student enrollment patterns are akin to a liquid taking the shape of its container, does it not commonsensically follow that the "shape" of the course schedule has to be modified commensurately in order to steer student "flow" toward off-peak time blocks?

- Is it counterintuitive that graduate-level mathematics classes are rarely scheduled in one-day-per-week formats, yet developmental-level mathematics classes often are? Said differently, if massed meetings are often not available to advanced mathematics students, why are they so prevalent with at-risk community college students?

- Is there a relationship between frequency of students' availing themselves of an institution's learning-resource-center (LRC) support facilities and the seat-time configuration of their course? For instance, is a part-time student who is taking a oneday-per-week, three-hour algebra course more, less, or just as likely to visit the LRC before/after class as the part-time student in a two- or three-day-per-week, shorterduration class?

- Some part-time working adult students, especially those who placed into one or more developmental-level courses, do not persist through their degree program due to how 
long they project it will take them to graduate when they are only taking one course per semester. For many of them, the climb feels steep, and their ascent slow. Therefore, might scheduling two-night-per-week classes in a coordinated, crossdisciplinary way help? For example, if most one-night-per-week developmentalalgebra courses were replaced with two-night-per-week course offerings and were paired with, say, a two-night-per-week developmental-level writing or reading class scheduled immediately before or after the algebra class, would not more part-time students be enticed to enroll in two courses per term, instead of just one? Might this enhance persistence rates? Furthermore, might this also create the opportunity for evening students to enjoy the cross-disciplinary, learning-community type course offerings that often have been limited to only daytime classes?

- Similarly, many 2-year colleges have large, if not administratively unwieldy, numbers of part-time faculty. Might it help streamline the size of a mathematics department's adjunct-instructor pool—and, hence, make it less managerially unwieldy—by offering more two-night-per-week courses (in consecutive back-to-back time blocks, such as 5:30-6:45, 7:00-8:15, 8:30-9:45) instead of one-night-per-week (e.g., 7:009:50) offerings? That is, would such a course-scheduling design decrease the number of part-time hires by assigning two or more back-to-back courses to a single instructor two nights per week, instead of assigning two different instructors to a single one-night-per-week section each?

- The flipped (or inverted) classroom instructional strategy dedicates in-class seat time for hands-on active learning activities, while relegating lecture-type instruction to online videos and other multimedia forms of supplemental materials for students to 
view outside of class. If, as some have claimed, this pedagogical approach continues to gain attention and becomes more prevalent (Bull, Ferster, \& Kjellstrom, 2012; Millman, 2012), what impact, if any, will it have upon seat-time apportionment best practices? How might this alter the way in which practitioners and researchers conceptualize and investigate cognitive theories such as the spacing effect, encodingvariability theory, and the new theory of disuse, especially in light of how few experimental studies of the spacing effect in the context of mathematics practice have been undertaken (Rohrer, 2009)? For instance, is it possible to define and quantify the spacing of learning episodes in a world in which classroom learning has become an asynchronous experience? If so, how?

\section{Recommendations for Institutions and Practitioners}

The previous section's very concrete recommendations pertaining to coursescheduling and enrollment-management practices segue naturally into several interrelated larger propositions.

America's community colleges pride themselves on their spirit of innovation and openness, both in terms of open-door outreach to their community's diverse, aspiring learners and in terms of open-arms responsiveness to their locality's workforce training needs. In recent years, with the addition of select 4-year baccalaureate degree programs at many institutions and more rapid changes in the labor market, the societal role of 2-year colleges has become even larger and more complex. Concurrently, while the impetus to innovate has increased sharply, so have the accountability demands upon 2-year colleges to improve their very low graduation rates. However, there are several crucial pieces of this complex puzzle that this researcher contends (a) have not been given ample attention 
and (b) are significant hindrances to reform initiatives aimed at increasing learning gains and success rates.

First, deficiencies in staffing and methodological expertise in many community college institutional research (IR) departments have been well documented (Achieving the Dream Community Colleges Count, 2005-2010; Levin \& Calcagno, 2008; Morest \& Jenkins, 2007; Romero et al., 2005). Levin and Calcagno (2008) proffered many valuable recommendations to help community colleges improve their institutional efforts to systematically evaluate academic programs; in particular, their remedial-level offerings. In light of the case made throughout much of this dissertation for improvements in research methodology and decision-making processes, the closing recommendation in Levin and Calcagno's scholarly paper merits a verbatim reiteration, for it fuses the assessment of other scholars (Morest \& Jenkins, 2007) with many of the decision-making methodological concerns examined in this researcher's dissertation:

... establish a central resource at the state level and cooperative efforts with universities to assist community colleges and individual faculty members in creating experimental interventions and to provide support for evaluating them. Standard intervention designs and data collection centers could be established as well as methods for analyzing data on outcomes and costs. Faculty members and administrators could collaborate with the evaluation staff inside or outside of their institutions to specify the appropriate outcomes and control variables, help administer the data instruments, and assist in the interpretation of the results. (p. 202) 
Secondly, the notion of increasing faculty engagement in such activities is particularly noteworthy. Morest and Jenkins (2007) found that, in general, 2-year college faculty are neither included among the participants in nor among the audience for IR studies. As Hardré (2012) observed, "Community college faculty members see lack of expertise and opportunities, as well as administrative support, as roadblocks to both basic research and teaching research activities, and these are elements that can be administratively addressed" (p. 558). Not only can they be administratively addressed, this researcher maintains they must be addressed. Otherwise, educational policymakers and 2-year college administrators and faculty, by and large, will continue to be incapable of properly evaluating initiatives, to assess which are working, specifically why they are working, and how (and where) to best institute and refine them. Without such knowledge, efforts to overcome faculty skepticism and acquire classroom-level buy-in will be impeded, all of which obviously thwarts the expansion of worthwhile innovations that may significantly benefit students.

Thirdly, there is an essential prerequisite that must be fulfilled before community college faculty and administrators can be motivated and equipped to actively engage in such activities, first as readers (and users) of research studies and ultimately as collaborators with IR professionals and others: they must be provided ongoing training, support, and encouragement to steadily increase their understanding of (and appreciation for) educational research methodology. To be most impactful, such an undertaking must be a high ongoing priority both trans-institutionally and intra-disciplinarily. One highly recommended resource that deserves widespread attention is Hardré (2012), whose study identified many important, under-examined aspects of this issue in regards to community 
college faculty and the institutional benefits of increasing faculty engagement in research activities. For instance, she emphasized the importance of faculty professional development opportunities being “...linked to intrinsic or extrinsic motivation and clearly connected to faculty perceived needs and interests. Otherwise, faculty not only will not be motivated but will resist and resent it" (p. 557).

The fourth recommendation echoes others' calls (e.g., Di Muro \& Terry, 2007; Jones et al., 2003) for devising means to increase faculty knowledge, awareness, and classroom application of relevant and robust theories of cognition, information processing, and social development, in tandem with better training in methods of learning assessment. This is, arguably, the most pressing priority, in terms of properly equipping 2-year college faculty—especially mathematics educators—with the pedagogical tools and heightened self-awareness needed to more effective in the classroom with today's increased diversity of students and learning-style predispositions.

As Evans et al. (2010) emphasized, "If academic disciplines are to be accessible to students with diverse learning styles, efforts must be made to provide varied methods of instruction and evaluation" (p. 143). All too often, though, algebraic concepts and other mathematical content are imparted in a traditional lecture-style manner that is largely comprised of rote memorization of rules and symbolic-manipulation procedures, with hardly any cognitive-process-friendly thematic organization. This is in part attributable to (a) the large disparity between the learning style predilections of most mathematics educators versus those of today's students (Di Muro \& Terry, 2007); and, more consequentially, (b) the fact that large numbers of mathematics teachers are unaware of this gap and approaches to bridging it. Research on Kolb's (1984) 
experiential learning model (ELM) has revealed that mathematics majors tend to be what are termed assimilators, those who are inclined toward abstract conceptualization and reflective observation and, consequently, prefer abstract notions, logical cogency, and contemplation over social interaction and collaboration (Kolb et al., 2001). However, community college students, especially those placed in remedial-level mathematics courses, tend to fall into the other three learning styles in Kolb's ELM; for example, the diametric opposite of assimilators, accommodators, who favor concrete experience and active experimentation.

With regard to how this recommendation ought to be implemented, one may choose to view it separately and independently from the preceding three recommendations; or, alternatively, as a precursor to- or, for that matter, integrated as a component of -research-related professional development for community college faculty. Furthermore, it may be undertaken as an institution-directed formal imperative or, in the absence of institutional support, as a faculty-led informal self-improvement program. Many resources exist in this regard; for instance, Di Muro and Terry (2007), the former a mathematics lecturer and the latter a student-services administrator, provided an easily digestible primer on the application of learning-style theory to mathematics instruction.

Fifthly, this researcher respectfully encourages graduate-level university faculty in the field of education to assess whether their programs' course offerings in quantitative research methods have failed to keep pace with advances in multi-level and longitudinal techniques of analysis. It is reasonable to speculate that part of the reason why education research has lagged so far behind many other fields of scholarly inquiry in embracing 
longitudinal quantitative methods (Singer \& Willett, 2004) is attributable to omissions in graduate-level curricula.

Finally, in light of the fact that one of this researcher's two overarching aims in this study was to contribute to improvements in the way student persistence and attrition are analyzed, it seems fitting to close this section by providing two data-related suggestions for colleges, universities, and education-related governmental agencies.

First, the analysis of this study's second research question brought to light a serious deficiency in using the course-withdrawal ("W") grade as a means by which to measure student attrition. Namely, various postsecondary institutions allow the "W" grade to be assigned to students in two ways: (a) student-initiated course withdrawals prior to the institution's withdrawal deadline, and (b) instructor-initiated withdrawals at any point throughout the semester, provided the instructor has self-acquired that right via the course syllabus. This conflation may have altered this study's findings, as the reliability of the "W" grade — and its affixed withdrawal date — as a measure of course attrition were compromised.

Some illustrations should clarify the many ways in which the "W" grade's integrity may be compromised. For example, at some institutions, instructors have the decision-making latitude, if ever and whenever desired, to manually withdraw a student before (as well as after) the student-initiated deadline. One instructor may have decided that a given student's violations of the instructor's class attendance policy early in the semester warranted immediate course withdrawal. Meanwhile, that instructor's departmental colleague may view the "W" as a "course registration status" instead of as a "grade." Hence, with this belief that assignment of the " $\mathrm{W}$ " grade is solely within the 
student's purview, that departmental colleague would likely assign an " $F$ " grade to a student who was chronically absent throughout the semester.

Additionally, consider this end-of-semester scenario: the instructor who, while submitting semester grades, circumvents the spirit of the institution's course-withdrawal policy by improperly granting "W" grades to hardworking students who performed well for much of the semester yet ultimately did not attain a passing course average. This type of practice has been known to occur, for example, among instructors of developmentallevel courses, some of whom have posited that, since the course does not count for college-level credit, students' transcripts should not be marred by failing "F" grades in these preparatory-type courses that are, for all intents and purposes, non-credit endeavors.

The larger point is this: commingling instructor-initiated and student-initiated course withdrawals by using the same grade-letter code (e.g., "W") for both greatly diminishes its internal consistency and, hence, its reliability as a measure of student attrition. This researcher, therefore, suggests institutions remedy this variable pollution by either creating two separate grade-letter codes or, alternatively, using a two-symbol approach (e.g., "W" for student-initiated withdrawal and "WI" for instructor-initiated). In the event that recent changes in federal-level financial-aid regulations preclude such a delineation from appearing on the student's permanent academic record, then it is recommended that institutions create an internal-use variable field within their studentrecords database system that will serve to aid two important constituencies: their internal institutional-research (IR) departments and the state-level agencies to which they report.

Like the "W" grade conflation problem, this researcher's second data-related recommendation pertains to another severe variable deficiency: the collection and use of 
race and ethnicity variables as student-level or instructor-level covariates. Simply stated, there is a troubling paradox: so much attention is paid to differences in achievement and attrition differences between race-ethnicity groups, yet so little attention has been given to the these demographic variables' high missing-value and low internal-consistency rates.

As was detailed in Chapter 4 (see Table 1 and related discussion), race and ethnicity had exorbitant missing-value rates, with the former being $26.2 \%$ and the latter $67.9 \%$. As the researcher learned via follow-up inquiries with the institution's registrar and IR staff and, in addition, via a review of National Center for Education Statistics (NCES) documents, race and ethnicity are self-reported, optional-response items, which are typically acquired during the college application/admissions process. In contrast, variables like immigration status and birth country have much lower unreported-value rates and higher reliability, due to the verification and documentation processes required of institutions.

These same data-collection shortcomings occurred with the Level-2 instructor demographic variables obtained for this study. In short, so many instructors opted to not share their race-ethnicity during the hiring process that these covariates were unable to be incorporated into this study's multilevel analyses.

Therefore, the researcher recommends that, within the obvious constraints of federal and state statutes and related regulations, institutions consider three corrective actions. First, re-assess their current collection approaches to race-ethnicity data and whether there are ways to reduce the non-response rates. For instance, investigate whether there is a significant improvement when community college students complete 
entry applications electronically versus on paper. Second, be forthcoming in all race- and ethnicity-related data reporting, with regard to: (a) disclosing the self-reporting, missingvalue, and other internal-consistency deficiencies in these variables; and (b) providing the appropriate caveats and disclaimers when stating findings and inferences. Third, and finally, strive to actively educate policymakers, legislators, accreditation agencies, and others of the statistical complications and dangers related to quantifying race/ethnicityrelated achievement and persistence, in light of the shortcomings in (a) how these measures are collected, and (b) their internal-consistency challenges, especially with respect to the increasing population of multi-ethnic, multi-racial students.

\section{Recommendations for Other Researchers}

This researcher contends that the topic of this study, as well as its methodology, opens the door to a wide array of future investigations.

First, as was discussed in Chapter 3, a number of current scholars have advanced our knowledge and understanding of the importance of incorporating institutional-context variables in student-persistence studies, with the aid of multi-level quantitative methods. Moreover, Titus (2004), for example, has demonstrated the pitfalls of judging institutional effectiveness on the basis of analyses that either omit or inappropriately include institutional-context factors. Extending Titus's contributions, Chen (2012) contends:

Future research is encouraged to further examine the effects of other institutional characteristics closely connected to students' experiences, such as peer environments, faculty cultures, and internal structural or policy considerations. Further empirical research is warranted before policy changes can be advocated 
for several reasons... [including the fact that]... research on what institutional characteristics matter in student dropout/persistence decisions is still very limited. (p. 501)

Therefore, future studies of seat-time apportionment that, in contrast to this dissertation's single-institution sample, utilize multi-institutional data sets and institutional-context covariates would be a significant advance of this research. Such an investigation would seem to be particularly well-suited to college systems like Florida's because: (a) all of the 28 2-year colleges in Florida share a common course-numbering system and very similar degree/program requirements; (b) a number of the institutions are part of a joint consortium which lends itself to the sharing of data; and (c) it is not uncommon for postsecondary students (particularly in larger urban areas like South Florida) to enroll in multiple Florida 2-year colleges, even simultaneously within the same semester.

Second, as was discussed on several occasions within the preceding chapters, it was a challenge to operationally define seat-time level in a way that would disentangle it from the different levels of course session and semester length (e.g., 16-week fullsemester course sections versus 8 -week mini-term offerings of the identical course within that same semester). Because of the dramatic increase in recent years of mini-session course offerings, it is recommended that future research adapt the methodological approaches applied in this seat-time study to the matter of session- and semester-length. That is to say, it is worthwhile to investigate whether changing the discrete-time survival analysis's (DTSA) grouping variable from seat time to session length alters the findings.

Third, it should be noted that this study employed only one of the two broad types of survival-analysis methodology. Specifically, the DTSA analysis of this study's second 
and third research questions was limited to first describing the proportion of students surviving at specific time intervals and then testing for group differences. This approach did not incorporate the assorted Level-1 and Level-2 predictors collected. Hence, followup studies to determine whether group differences exist after statistically controlling for these covariates are strongly encouraged.

Fourth, it is important to remember that, in the case of the two-year longitudinal tracking of this study's third research question, the DTSA time metric was tied to every student's Fall-2001 MAT0024 attempt; regardless of (a) whether the student passed the course in that semester or not, and (b) whether that Fall-2001 course experience was the student's first attempt at MAT0024 or second or third, and so on. Hence, a future DTSA study should consider re-defining the survival-event's start time in several alternative, and potentially better, ways. These include: defining the start time as the end of the term in which the student has successfully completed MAT0024 and then tracking forward from that point in time; or, instead, defining the start time as the end of the term in which the student's first MAT0024 attempt occurred, regardless of whether the student was successful in the course or not.

Fifth, future studies should include additional variables that can affect student success but were not taken into account in this study, such as: pedagogical differences (e.g., traditional lecture versus group work); differences in types and frequency of assessments; whether there is course exit-exam and, if so, at what level (departmentwide, college-wide, state-wide) and whether a passing score on the exit exam is a necessary condition to earning credit for the course; and differences in homework tasks (e.g., assignment types, quantity, frequency, and weight in course grade computation). 
In closing, it is this researcher's desire that this multi-level, longitudinal study of student success/persistence in community-college algebra courses has provided a useful methodological template, albeit a draft-version one, that future studies can refine and customize in countless many ways. Possible refinements and customizations range from explorations of student achievement and perseverance in other academic disciplines (e.g., developmental-level reading or developmental-level writing) to investigations that longitudinally track students in ways that are not in any way discipline-specific (e.g., time to complete a given number of credit hours or time to complete a given degree program).

In their examination of community college institutional research (IR) departments, Morest and Jenkins (2007) found:

[Although] performing longitudinal analysis using student cohort data... is essential for well-conceived data-based decision making... at many colleges the capacity for this kind of analytical research does not exist. However, even in those instances where it does exist, IR personnel rarely, if every [sic], carry out sophisticated analyses for use in college management or in efforts to improve programs and services. (p. 11)

A goal of this dissertation study has been to contribute to others' efforts to change that. 


\section{References}

Achieving the Dream Community Colleges Count. (2005-2010). Community College Strategies. Retrieved June 2, 2010, from Achieving the Dream:

http://www.achievingthedream.org/CAMPUSSTRATEGIES/GUIDES/default.tp

Andreu, M. L. (2002). Developing and implementing local-level retention studies: A challenge for community-college institutional researchers. Community College Journal of Research and Practice, 26(4), 333-344.

Aronson, J. (2004). The threat of stereotype. Educational Leadership, 62(3), 14-19.

Baddeley, A. D. (1976). The psychology of memory. New York, NY: Basic Books.

Bailey, T., Jeong, D. W., \& Cho, S. W. (2010). Referral, enrollment, and completion in developmental education sequences in community colleges. Economics of Education Review, 29(2), 255-270.

Berk, R. (2005). Randomized experiments as the bronze standard. Los Angeles, CA: Department of Statistics, UCLA. Retrieved from http://escholarship.org/uc/item/2d57d1kq

Biemiller, L. (2009, March 27). Bursting at the seams. The Chronicle of Higher Education, pp. B11-B13.

Bini, M., Monari, P., Piccolo, D., \& Salmaso, L. (Eds.). (2009). Statistical methods for the evaluation of educational services and quality of products. Heidelberg, Germany: Springer.

Bjork, R. A., \& Bjork, E. L. (1992). A new theory of disuse and an old theory of stimulus fluctuation. In A. Healy, S. Kosslyn, \& R. Shiffrin (Eds.), From learning processes to cognitive processes: Essays in honor of William K. Estes (Vol. 2, pp. 35-67). Hillsdale, NJ: Erlbaum.

Bjork, R. A., Dunlosky, J., \& Kornell, N. (2013). Self-regulated learning: Beliefs, techniques, and illusions. Annual Review of Psychology, 64, 417-444. doi:10.1146/annurev-psych-113011-143823

Brown II, M. C., Butler, J. L., \& Donahoo, S. (2004). Desegration and diversity: Finding new ways to meet the challenge. In E. P. St. John, \& M. D. Parsons (Eds.), Public funding of higher education: Changing contexts and new rationales (pp. 108123). Baltimore, MD: The Johns Hopkins University Press. 
Budé, L., Imbos, T., van de Wiel, M. W., \& Berger, M. P. (2011). The effect of distributed practice on students' conceptual understanding of statistics. Higher Education, 62(1), 69-79.

Bugay, D. P. (2000). The five-week class of the University of Phoenix corporate model can be utilized by community colleges. (Doctoral dissertation). The Union Institute - Ohio. (UMI No. 9989085).

Bull, G., Ferster, B., \& Kjellstrom, W. (2012, August). Inventing the flipped classroom. Learning \& Leading with Technology, 40(1), 10-11.

Burns, K. (2010). Community college student success variables: A review of the literature. Community College Enterprise, 16(2), 33-61.

Carpenter, S. K., Cepeda, N. J., Rohrer, D., Kang, S. H., \& Pashler, H. (2012). Using spacing to enhance diverse forms of learning: Review of recent research and implications for instruction. Educational Psychology Review, 24(3), 369-378.

Cepeda, N. J., Vul, E., Rohrer, D., Wixted, J. T., \& Pashler, H. (2008). Spacing effects in learning: A temporal ridgeline of optimal retention. Psychological Science, 19(11), 1095-1102.

Chen, R. (2012). Institutional characteristics and college student dropout risks: A multilevel event history analysis. Research in Higher Education, 53(5), 487-505.

Chen, R., \& St. John, E. P. (2011). State financial policies and college student persistence: A national study. The Journal of Higher Education, 82(5), 629-660.

Collins, L. M., \& Sayer, A. G. (Eds.). (2001). New methods for the analysis of change. Washington, DC: American Psychological Association.

Complete College America. (2010). Boosting completion at community colleges: Time, choice, structure, and the significant role of states. The White House Working Group for The President's Summit on Community Colleges. Washington, DC: Complete College America.

Complete College America. (2011, October 7). Florida 2011. Retrieved from Complete College America: http://www.completecollege.org/docs/Florida.pdf

Complete College America. (2012). Remediation: Higher education's bridge to nowhere. Washington, DC: Complete College America. Retrieved from http://www.completecollege.org/docs/CCA-Remediation-final.pdf

Cuyjet, M. J. (2006). African American men in college. San Francisco, CA: Jossey-Bass. 
Daniel, E. L. (2000). A review of time-shortened courses across disciplines. College Student Journal, 34(2), 298-308.

Dempster, F. N. (1988). The spacing effect: A case study in the failure to apply the results of psychological research. American Psychologist, 43(8), 627-634.

Deil-Amen, R., \& Rosenbaum, J. E. (2002). The unintended consequences of stigma-free remediation. Sociology of Education, 75(3), 249-268.

Di Muro, P., \& Terry, M. (2007). A matter of style: Applying Kolb's learning style model to college mathematics teaching practices. Journal of College Reading and Learning, 38(1), 53-60.

Ebbinghaus, H. (1964). Memory: A contribution to experimental psychology. (H. A. Ruger, \& C. E. Bussenius, Trans.) New York, NY: Dover Publications. (Original work published 1885).

Evans, N. J., Forney, D. S., Guido, F. M., Patton, L. D., \& Renn, K. A. (2010). Student development in college: Theory, research, and practice (2nd ed.). San Francisco, CA: Jossey-Bass.

Felder, R. M., \& Brent, R. (2005, January). Understanding student differences. Journal of Engineering Education, 94(1), 57-72.

Fike, D. S., \& Fike, R. (2008). Predictors of first-year student retention in the community college. Community College Review, 36(2), 68-88.

Florida Division of Community Colleges. (2002, February). The fact book: Report for the Florida community college system. Retrieved September 24, 2012, from FLDOE Hub:

http://www.fldoehub.org/CCTCMIS/c/Documents/Fact\%20Books/webversion.pd $\mathrm{f}$

Florida State Board of Community Colleges. (1998, August 17). Department of Education. Retrieved May 12, 2010, from http://www.fldoe.org/CC/policy/pol23.asp?style=print

Fritz, C. O., Morris, P. E., Bjork, R. A., Gelman, R., \& Wickens, T. D. (2000). When further learning fails: Stability and change following repeated presentation of text. British Journal of Psychology, 91, 493-511.

Fuchs, A. H. (1997). Ebbinghaus's contributions to psychology after 1885. The American Journal of Psychology, 110(4), 621-633. 
Gallo, M. A., \& Odu, M. (2009). Examining the relationship between class scheduling and student achievement in college algebra. Community College Review, 36(4), 299-325.

Goble, L. J., Rosenbaum, J. E., \& Stephan, J. L. (2008). Do institutional attributes predict individuals' degree success at two-year colleges? New Directions for Community Colleges, 2008(144), 63-72.

Hagedorn, L. S., Maxwell, W. E., Cypers, S., Moon, H. S., \& Lester, J. (2007). Course shopping in urban community colleges: An analysis of student drop and add activities. Journal of Higher Education, 78(4), 464-485.

Hardré, P. L. (2012). Community college faculty motivation for basic research, teaching research, and professional development. Community College Journal of Research and Practice, 36(8), 539-561.

Heck, R. H., Thomas, S. L., \& Tabata, L. N. (2010). Multilevel and longitudinal modeling with IBM SPSS. New York, NY: Routledge/Taylor \& Francis Group.

Heck, R. H., Thomas, S. L., \& Tabata, L. N. (2012). Multilevel modeling of categorical outcomes using IBM SPSS. New York, NY: Routledge Taylor \& Francis Group.

Hilgard, E. R. (1964). Introduction to Dover edition. In H. Ebbinghaus, Memory: A contribution to experimental psychology (H. A. Ruger, \& C. E. Bussenius, Trans., pp. vii-x). New York, NY: Dover Publications.

Hinkle, D. E., Wiersma, W., \& Jurs, S. G. (2003). Applied statistics for the behavioral sciences (5th ed.). Boston, MA: Houghton Mifflin Company.

Hispanic Association of Colleges \& Universities. (2012, September 21). Hispanic Association of Colleges and Universities - HACU. Retrieved September 21, 2012, from Hispanic Association of Colleges and Universities: http://www.hacu.net/assnfe/CompanyDirectory.asp?STYLE=2\&COMPANY_TY $\mathrm{PE}=1,5 \& \mathrm{SEARCH}$ TYPE$=0$

Hosmer, D. W., \& Lemeshow, S. (2000). Applied Logistic Regression (2nd ed.). New York, NY: John Wiley \& Sons, Inc.

Hosmer, D. W., Lemeshow, S., \& May, S. (2008). Applied survival analysis: Regression modeling of time-to-event data (2nd ed.). Hoboken, NJ: John Wiley \& Sons, Inc.

Hox, J. J. (2010). Multilevel analysis: Techniques and applications (2nd ed.). New York, NY: Routledge. 
Jones, C., Reichard, C., \& Mokhtari, K. (2003, June). Are students' learning styles discipline specific? Community College Journal of Research and Practice, 27(5), 363-375.

Kerlinger, F. N., \& Lee, H. B. (2000). Foundations of behavioral research (4th ed.). Fort Worth, TX: Harcourt College Publishers.

Knapp, L. G., Kelly-Reid, J. E., \& Ginder, S. A. (2012). Enrollment in postsecondary institutions, fall 2010; financial statistics, fiscal year 2010; and graduation rates, selected cohorts, 2002-07 (NCES 2012-280). U.S. Department of Education. Washington, DC: National Center for Education Statistics. Retrieved September 28, 2012, from http://nces.ed.gov/pubsearch/pubsinfo.asp?pubid=2012280

Kolb, D. A. (1984). Experiential learning: Experience as the source of learning and development. Saddle River, NJ: Prentice Hall.

Kolb, D. A., Boyatzis, R. E., \& Mainemelis, C. (2001). Experiential learning theory: Previous research and new directions. In R. J. Sternberg, \& L-F. Zhang (Eds.), Perspectives on cognitive, learning, and thinking styles (pp. 193-210). Mahwah, NJ: Lawrence Erlbaum Associates.

Kornell, N., \& Bjork, R. A. (2008). Learning concepts and categories: Is spacing the "enemy of induction"? Psychological Science, 19(6), 585-592.

Kucsera, J. V., \& Zimmaro, D. M. (2010). Comparing the effectiveness of intensive and traditional courses. College Teaching, 58(2), 62-68.

Kuh, G. D., Cruce, T. M., Shoup, R., Kinzie, J., \& Gonyea, R. M. (2008, Sept/Oct). Unmasking the effects of student engagement on first-year college grades and persistence. The Journal of Higher Education, 79(5), 540-563.

Lang, E. (2009). Sustaining enrollment growth in difficult financial times. Diverse Issues in Higher Education, 26(13), 14.

Lazari, A. (2007). Does the number of lectures a week make a difference in the learning concepts and retention of students in college algebra? Georgia Journal of Science, 65(2), 72-75.

Levin, H. M., \& Calcagno, J. C. (2008). Remediation in the community college: An evaluator's perspective. Community College Review, 35(3), 181-207.

Lillibridge, F. (2008). Retention tracking using institutional data. New Directions for Community Colleges, 143, 19-30. 
Little, T. D., Schnabel, K. U., \& Baumert, J. (Eds.). (2000). Modeling longitudinal and multilevel data: Practical issues, applied approaches, and specific examples. Mahwah, NJ: Lawrence Erlbaum Associates.

MacCallum, R. C., \& Kim, C. (2000). Modeling multivariate change. In T. D. Little, K. U. Schnabel, \& J. Baumert (Eds.), Modeling longitudinal and multilevel data: Practical issues, applied approaches, and specific examples (pp. 51-68). Mahwah, NJ: Lawrence Erlbaum Associates.

Mangan, K. (2008, May 30). Longer days and shorter weeks as colleges cope with soaring energy costs. The Chronicle of Higher Education, p. A16.

Mercer, C. J., \& Stedman, J. B. (2008). Minority-serving institutions: Selected institutional and student characteristics. In M. Gasman, B. Baez, \& C. S. Turner (Eds.), Understanding minority-serving institutions (pp. 28-42). Albany, NY: State University of New York Press.

Milman, N. B. (2012). The flipped classroom strategy: What is it and how can it best be used? Distance Learning, 9(3), 85-87.

Mook, D. (2004). Classic experiments in psychology. Westport, CT: Greenwood Press.

Moosai, S., Walker, D. A., \& Floyd, D. L. (2011). Using student and institutional characteristics to predict graduation rates at community colleges: New developments in performance measures and institutional effectiveness. Community College Journal of Research and Practice, 35(10), 802-816.

Morest, V. S., \& Jenkins, D. (2007). Institutional research and the culture of evidence at community colleges. Columbia University, Institute on Education and the Economy, Teachers College. New York, NY: Community College Research Center.

Morgan, S. L., \& Winship, C. (2007). Counterfactuals and causal inference: Methods and principles for social research. New York, NY: Cambridge University Press.

Mullens, J. E., Murnane, R. J., \& Willett, J. B. (1996, May). The contribution of training and subject matter knowledge to teaching effectiveness: A multilevel analysis of longitudinal evidence from Belize. Comparative Education Review, 40(2), 139157.

Mullin, C. M., \& Phillippe, K. (2011). Fall 2011: Estimated headcount enrollment and Pell grant trends. Washington, DC: American Association of Community Colleges. 
Murnane, R. J., \& Willett, J. B. (2011). Methods matter: Improving causal inference in educational and social science research. New York, NY: Oxford University Press.

Nelson Laird, T. F., \& Cruce, T. M. (2009). Individual and environmental effects of parttime enrollment status on student-faculty interaction and self-reported gains. Journal of Higher Education, 80(3), 290-314.

Newman, I., \& Benz, C. R. (1998). Qualitative-quantitative research methodology: Exploring the interactive continuum. Carbondale, IL: Southern Illinois University Press.

Odu, M. (2008). Class scheduling in college algebra: An examination of the spacing effect on student achievement. (Doctoral dissertation). Florida Institute of Technology. (UMI No. 3303348).

Okpala, C. O., Hopson, L., \& Okpala, A. O. (2011, March). The impact of current economic crisis on community colleges. College Student Journal, 45(1), 214-216.

Pahl, K., \& Way, N. (2006, Sep-Oct). Longitudinal trajectories of ethnic identity among urban black and latino adolescents. Child Development, 77(5), Special Issue on Race, Ethnicity, and Culture in Child Development, 1403-1415.

Perrakis, A. I. (2008). Factors promoting academic success among African-American and White male community college students. New Directions for Community Colleges, 2008(142), 15-23.

Peterson, M. W., \& Dill, D. D. (1999). Understanding the competitive environment of the postsecondary knowledge industry. In J. L. Bess, \& D. S. Webster (Eds.), ASHE reader series: Foundations of American higher education (2nd ed., pp. 508-525). Boston, MA: Pearson Custom Publishing.

Pew Research Center. (2009, October). College enrollment hits all-time high, fueled by community-college surge. Washington, DC: Author.

Raudenbush, S. W., \& Bryk, A. S. (2002). Hierarchical linear models: Applications and data analysis methods (2nd ed.). Thousand Oaks, CA: Sage Publications.

Reason, R. D. (2009). Student variables that predict retention: Recent research and developments. NASPA Journal, 46(3), 482-501. (Reprinted from NASPA Journal, 2003, 40(4), pp. 172-191).

Rohrer, D. (2009). The effects of spacing and mixing practice problems. Journal for Research in Mathematics Education, 40(1), 4-17. 
Rohrer, D., \& Taylor, K. (2006). The effects of overlearning and distributed practise on the retention of mathematics knowledge. Applied Cognitive Psychology, 20, 12091224.

Romero, M., Purdy, L., Rodriquez, L., \& Richards, S. (2005). Research needs and practices of community-college practitioners. Community College Journal of Research and Practice, 29(4), 289-302.

Rosenbaum, J. E. (2011). The complexities of college for all : Beyond fairy-tale dreams. Sociology of Education, 84(2), 113-117.

Rosenbaum, J. E., Redline, J., \& Stephan, J. L. (2007). Community college: The unfinished revolution. Issues in Science and Technology, 23(4), 49-56.

Ruspini, E. (2002). Introduction to longitudinal research. New York, NY: Routledge.

Sanders, K. (2011, June 13). Gov. Rick Scott Says State Debt Is \$23 Billion. (J. Bartosek, Ed.) St. Petersburg Times, p. n/a (online newspaper edition).

Schneider, D. M. (1997). The spacing effect: Implications for relearning. (Doctoral dissertation). Rice University. (UMI No. 9727601).

Shadish, W. R., Cook, T. D., \& Campbell, D. T. (2002). Experimental and quasiexperimental designs for generalized causal inference. Boston, MA: Houghton Mifflin.

Singer, J. D., \& Willett, J. B. (2003). Applied longitudinal data analysis: Modeling change and event occurrence. New York, NY: Oxford University Press.

Sitzmann, T., \& Ely, K. (2011). A meta-analysis of self-regulated learning in workrelated training and educational attainment: What we know and where we need to go. Psychological Bulletin, 137(3), 421-442.

Slavin, R. E. (2010). Experimental studies in education. In B. P. Creemers, L. Kyriakides, \& P. Sammons, Methodological advances in educational effectiveness research (pp. 102-114). New York, NY: Routledge.

Snijders, T. A. B. (2005). Power and sample size in multilevel linear models. In B. S. Everitt, \& D. C. Howell (Eds.), Encyclopedia of statistics in behavioral science (Vol. III, pp. 1570-1573). Hoboken, NJ: John Wiley \& Sons.

Stasz, C., \& Bodilly, S. (2004). Efforts to improve the quality of vocational education in secondary schools: Impact of federal and state policies. Washington, DC: U.S. Department of Education. 
State Board of Education. (1998/2000/2004). Rule 6A-14.0301. Withdrawal and Forgiveness. Florida Administrative Code.

Syed, M., Azmitia, M., \& Cooper, C. R. (2011). Identity and academic success among underrepresented ethnic minorities: An interdisciplinary review and integration. Journal of Social Issues, 67(3), 442-468.

Tabachnick, B. G., \& Fidell, L. S. (2007). Using multivariate statistics (5th ed.). Boston, MA: Pearson Education.

Tallent-Runnels, M. K., Thomas, J. A., \& Lan, W. Y. (2006). Teaching courses online: A review of the research. Review of Educational Research, 76(1), 93-135.

Texas Higher Education Coordinating Board. (2005). Rule 13.105. Limitation on Formula Funding for Repeated Hours for Attempted Courses. Texas Administrative Code.

Titus, M. A. (2004). An examination of the influence of institutional context on student persistence at 4-year colleges and universities: A multilevel approach. Research in Higher Education, 45(7), 673-699.

Toppino, T. C., \& Bloom, L. C. (2002). The spacing effect, free recall, and two-process theory: A closer look. Journal of Experimental Psychology: Learning, Memory, and Cognition, 28(3), 437-444.

Tulving, E. (1985). Ebbinghaus's memory: What did he learn and remember? Journal of Experimental Psychology: Learning, Memory, and Cognition, 11(3), 485-490.

U.S. Department of Education. (2005, September 19). Archived: Secretary Spellings Announces New Commission on the Future of Higher Education. Retrieved from http://www2.ed.gov/news/pressreleases/2005/09/09192005.html

U.S. Department of Education. (2010a, August 25). OCR: Accredited Postsecondary Minority Institutions. Retrieved September 21, 2012, from U.S. Department of Education: http://www2.ed.gov/about/offices/list/ocr/edlite-minorityinst-listtab.html

U.S. Department of Education. (2010b, August 25). ED: Accredited Postsecondary Minority Institutions (Hispanic). Retrieved September 21, 2012, from U.S. Department of Education: http://www2.ed.gov/about/offices/list/ocr/edliteminorityinst-list-hisp-tab.html 
Willett, J. B., \& Singer, J. D. (2004, November 12). Longitudinal Research: Present Status \& Future Prospects. Retrieved June 5, 2010, from Harvard Graduate School of Education - Applied Longitudinal Data Analysis:

http://gseacademic.harvard.edu/alda/Presentations/Longitudinal\%20Research.ppt

Wilson, R. (2010, February 12). For-profit colleges change higher education's landscape: Nimble companies gain a fast-growing share of enrollments. The Chronicle of Higher Education, pp. A1, A16-A19. 


\section{Appendix A}

\section{FIU] INTERNATIONAL UNIVERSITY}

Office of Research Integrity Research Compliance, MARC 270

\section{MEMORANDUM}

To:

Steven Roig-Watnik

$\mathrm{CC}$ :

Dr. Benjamin Baez

File

From:

Jada Dixon, MJ, MPH, IRB Coordinator 9

Date: $\quad$ May 8, 2012

Re: Human Subject Research (HSR) Determination

Non-HSR Determination \# NHSD12-009

The project entitled, " "A Retrospective-Longitudinal Examination of the Relationship between Apportionment of Seat Time in Community-College Algebra Courses and Student Academic Performance"" has been carefully reviewed by the Office of Research Integrity. Based on the documents submitted, it has been determined that your project does not meet the definition of human subject research. Therefore, your project will not require the submission to and approval of the FIU Institutional Review Board (IRB).

Research means a systematic investigation, including research development, testing and evaluation, designed to develop or contribute to generalizable knowledge.

Human subject means a living individual about whom an investigator (whether professional or student) conducting research obtains (1) Data through intervention or interaction with the individual, or (2) Identifiable private information.

For further IRB information, please visit http://research.fiu.edu/compliance/compliance $h$ tml. 


\section{Appendix B}

Office of the Vice President for

Academic Affairs

Willis Holcombe Center

Phone 954-201-7426/Fax 954-201-7576

พIUS HOLCOMaE CENT

121 East Les Clas Blod

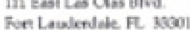

INSTITUTE FOR

ECONOMIC DEVELOPMENT

III Ear Los das Bund.

January 15, 2011

A. HUCH ADANS

CENTIAL CAMERUS

100 SW. Davie kas

Darke, HL. 20194

NOMTI CAMPUS

wow Coconut Cieck bivid

Cocumul Cred, Fl. 39066

JLDSON A SAMUELS

SOUTH CAMrLS

Troo Hollywosd/Tines Hived

Fentrobie Fines. Ft, ssot

PRES CENTER

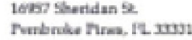

WESTON CENTRR 4ass bonwerfure Fond Westan, F. 33093

MURANAR AUTOMOTIVE

MaRUNe CENTIX

785 latien Bivad

Miramix F $x$ xom

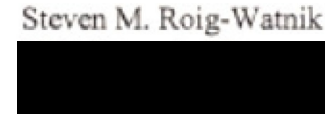

Dear Mr. Roig-Watnik:

Per your request and as required by Florida International University's Institutional Review Board (IRB), the purpose of this letter is to affirm that Broward College agrees to provide you access to the student-records data needed to conduct your doctoral dissertation study, pertaining to the relationship between apportionment of seat time in community-college algebra courses and student success.

The College and you have agreed that your research will not entail any contact with students nor proposed treatments for the student subjects in your research plan. Furthermore, in compliance with the Family Educational Rights to Privacy Act (FERPA), this letter confirms your agreement/understanding that you will not in any way use, share, nor publish any personally identifiable student information pertaining to any one person or multiple persons - neither within nor outside of the College - at any time during (and after the completion of) your research.

We wish you great success with this scholarly endeavor.

MULAMAL TOWN CENTER 2000 Cvik Ceneer Hace
Marumas F. sous

THGERTALL LAKE CENTE

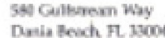

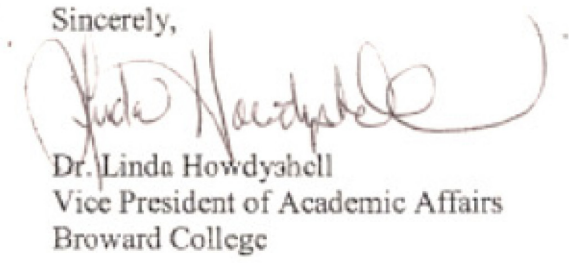

AN EQUAL ACLSE:EOUAT OTOWTUNITY RSTITUMON 
VITA

\title{
STEVEN MICHAEL ROIG-WATNIK
}

\author{
Born, Bronx, New York
}

\begin{tabular}{|c|c|}
\hline 1986 & $\begin{array}{l}\text { B.A., Mathematics } \\
\text { Florida Atlantic University } \\
\text { Boca Raton, FL }\end{array}$ \\
\hline 1989 & $\begin{array}{l}\text { M.A., Mathematics } \\
\text { The University of Texas at Austin } \\
\text { Austin, TX }\end{array}$ \\
\hline 1986-1987 & $\begin{array}{l}\text { Mathematics/Computer Teacher } \\
\text { Northeast High School } \\
\text { Oakland Park, FL }\end{array}$ \\
\hline 1986-1987 & $\begin{array}{l}\text { SAT Prep Math Teacher } \\
\text { Fort Lauderdale High School } \\
\text { Fort Lauderdale, FL }\end{array}$ \\
\hline 1986-1991 & $\begin{array}{l}\text { Private Tutor } \\
\text { Self-Employed }\end{array}$ \\
\hline $1987-1989$ & $\begin{array}{l}\text { Adult Computer Teacher } \\
\text { Cook Community School } \\
\text { Austin, TX }\end{array}$ \\
\hline 1989-1992 & $\begin{array}{l}\text { Mathematics Teacher } \\
\text { Ely High School } \\
\text { Pompano Beach, FL }\end{array}$ \\
\hline 1990-1992 & $\begin{array}{l}\text { Adjunct Instructor } \\
\text { Florida Atlantic University } \\
\text { Boca Raton, FL }\end{array}$ \\
\hline 1990-1992 & $\begin{array}{l}\text { Math Specialist } \\
\text { Homework Hotline } \\
\text { School Board of Broward County, FL }\end{array}$ \\
\hline $\begin{array}{l}1992-2000 \\
2000-2003\end{array}$ & $\begin{array}{l}\text { Assistant Professor of Mathematics } \\
\text { Associate Professor of Mathematics } \\
\text { Broward Community College } \\
\text { Pembroke Pines, FL }\end{array}$ \\
\hline
\end{tabular}


2003-2005

Mathematics Department Chairperson/Associate Dean

Broward Community College

Davie, FL

2005-present

Associate Professor of Mathematics

Broward College

Pembroke Pines, FL

\section{PUBLICATIONS}

Watnik, S.M. (2001). Student solutions manual for the nature of mathematics (9th ed.). Pacific Grove, CA: Brooks/Cole-Wadsworth Group.

Watnik, S.M. (2004). Student survival and solutions manual for the nature of mathematics (10th ed.). Belmont, CA: Brooks/Cole-Thomson Learning.

Watnik, S.M. (2004). Student survival and solutions manual for the nature of problem solving in algebra. Belmont, CA: Brooks/Cole-Thomson Learning.

Watnik, S.M. (2004). Student survival and solutions manual for the nature of problem solving in geometry and probability. Belmont, CA: Brooks/Cole-Thomson Learning. 